\title{
Tuning the Reactivity of Cofacial Porphyrin Prisms for Oxygen Reduction Using Modular Building Blocks
}

Matthew R. Crawley, Daoyang Zhang, Amanda N. Oldacre, Christine M. Beavers, Alan E. Friedman, and Timothy R. Cook*

\section{Supporting Information}

\section{Table of Contents}

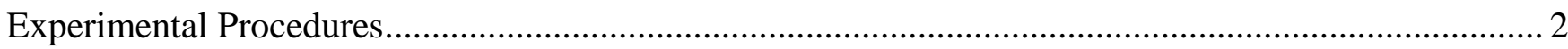

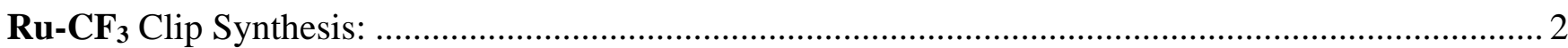

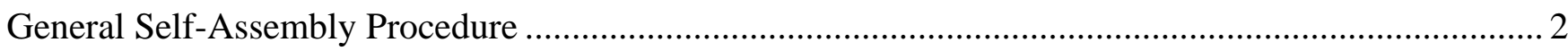

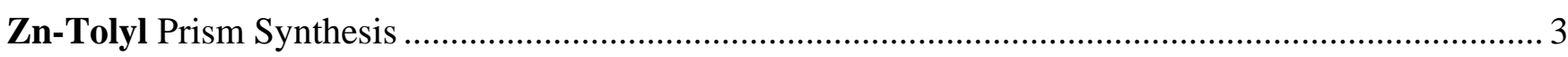

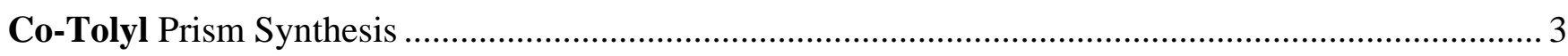

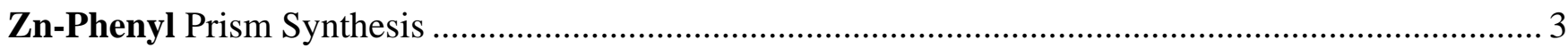

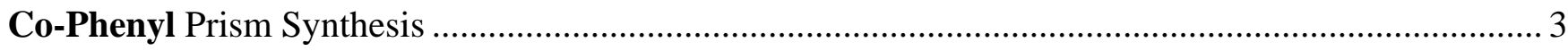

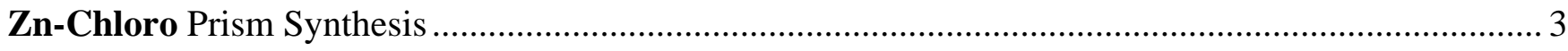

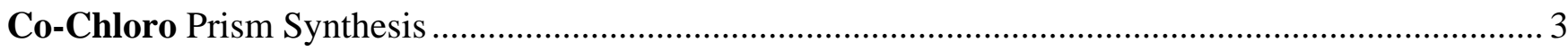

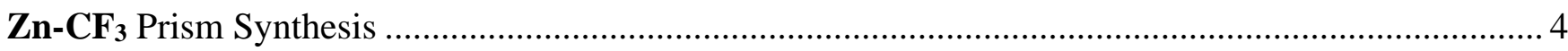

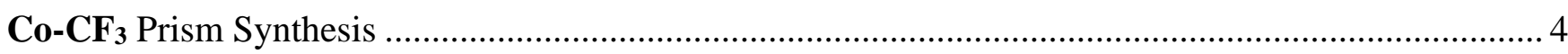

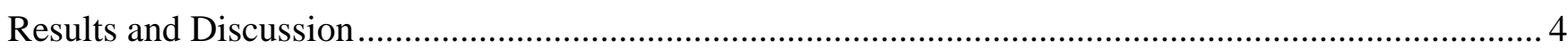

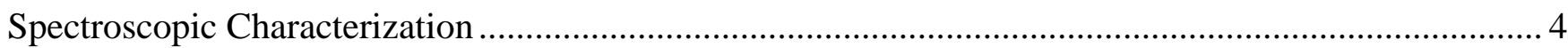

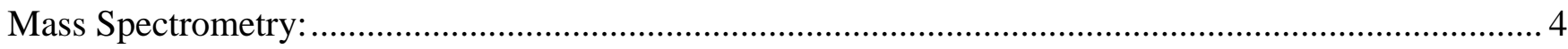

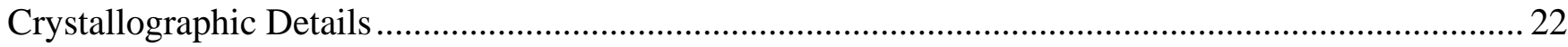

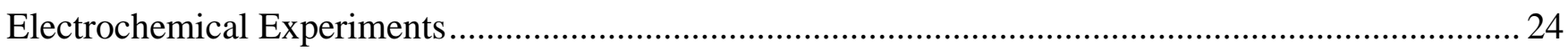

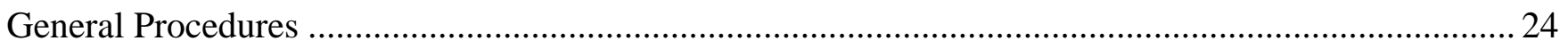

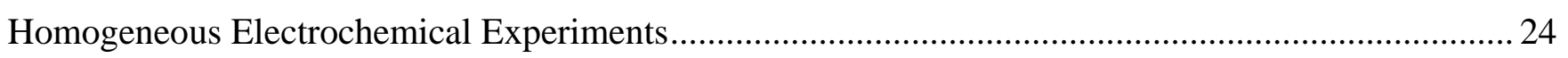

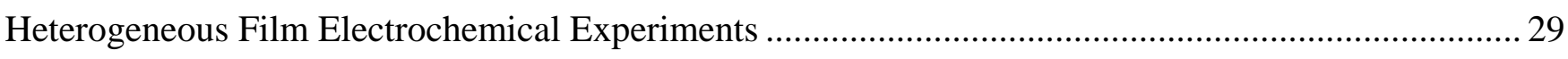

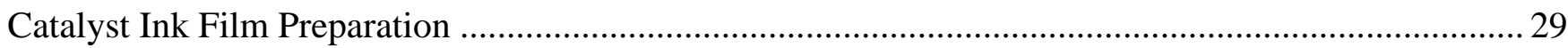

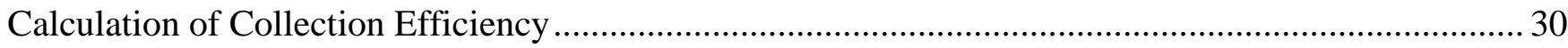

Faradaic Efficiency Determined using RRDE of Heterogeneous Catalyst Inks ......................................... 31

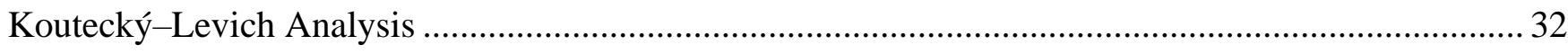

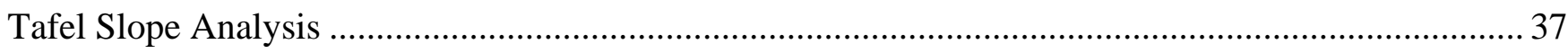

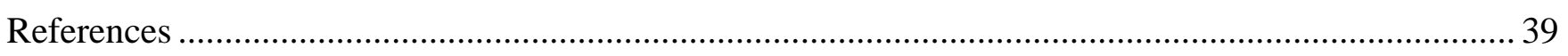




\section{Experimental Procedures}

Solvents were purified using a Grubbs style solvent system (Pure Process Technology). ${ }^{1} \mathrm{H}$ NMR spectra were acquired on Varian 300, 400, or $500 \mathrm{MHz}$ spectrometers. Chemical shifts $(\delta)$ were reported in parts per million (ppm) and referenced against the residual proteo-solvent peak in ${ }^{1} \mathrm{H}$. Samples were infused by electrospray Ionization using a home built ESI sprayer into a Bruker SolariX 12T FTMS (12 Tesla FT-ICR) system equipped with a dual source. In a typical experiment, twenty spectra were collected from $300-3000 \mathrm{~m} / \mathrm{z}$ at high resolving power $(\Delta=150,000 \mathrm{at} \mathrm{m} / \mathrm{z} 622$, where $\Delta$ is the peak full width at half-maximum peak height). Internal calibration mass accuracy results in an RMS mass error typically less than 500 ppb. The Ru-Tolyl, Ru-Phenyl, and Ru-Chloro clips were synthesized according to literature protocol using Ru-p-cymene chloro dimer purchased from Alfa Aesar or Strem Chemicals. ${ }^{1}$ ZnTPyP was purchased Frontier Scientific as used without further purification. CoTPyP was synthesized from TPyP (Frontier Scientific) following literature protocol. ${ }^{2}$ Silver triflate was purchased from Strem Chemicals and used as received. Methanol (HPLC grade, Fischer) was used as received without further drying or degassing. All self-assembly reactions were performed in air.

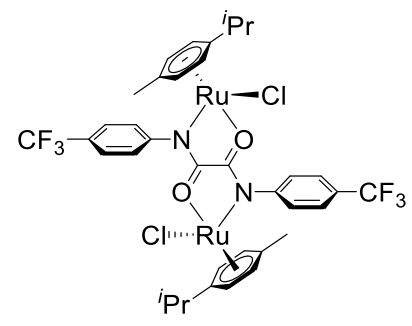

\section{$\boldsymbol{R u}-\boldsymbol{C F}_{3}$ Clip Synthesis:}

$N, N^{\prime}$-Bis(4-trifluoromethylphenyl)oxamide $\left(51 \mathrm{mg}, 0.14 \mathrm{mmol}, 1.0\right.$ eq.) was added to $15 \mathrm{~mL}$ of ethanol, and $\mathrm{Et}_{3} \mathrm{~N}(0.05 \mathrm{~mL}$, $0.4 \mathrm{mmol}, 2.5 \mathrm{eq}$.) was added slowly under a $\mathrm{N}_{2}$ atmosphere. Upon complete addition, the mixture was stirred for 30 minutes at $50^{\circ} \mathrm{C}$. The $\left(\eta^{6}-p \text {-cymene }\right)_{2} \mathrm{Ru}_{2}\left(\left(\mu_{2}-\mathrm{Cl}\right)_{2} \mathrm{Cl}_{2}\right)(100 \mathrm{mg}, 0.16 \mathrm{mmol}, 1.2 \mathrm{eq}$.$) was then added, and the mixture was refluxed$ overnight. The mixture was then allowed to cool to room temperature, the solvent volume was reduced to half in vacuo, and the mixture was placed in the freezer for 30 minutes. The orange solid was collected by filtration and washed with cold ethanol, acetone, ether, and pentane. The residue was dissolved in $\mathrm{CHCl}_{3}$, filtered through a Celite pad. An orange solution was collected and concentrated in vacuo, which yielded the desired product as orange solid. Single crystals suitable for X-ray diffraction were grown by slow evaporation from a saturated solution of the title compound in $\mathrm{CHCl}_{3}$. Yield (78.5 mg, 64.2\%). ${ }^{1} \mathrm{H}$ NMR $\left(300 \mathrm{MHz}, \mathrm{CDCl}_{3}\right): \delta=7.61\left(J_{\mathrm{H}, \mathrm{H}}=9 \mathrm{~Hz}, 6 \mathrm{H} ; \mathrm{Ar}-\mathrm{H}\right), 5.19\left(\mathrm{~d}, J_{\mathrm{H}, \mathrm{H}}=6 \mathrm{~Hz}, 2 \mathrm{H} ; \mathrm{Ar}-\mathrm{H}\right), 5.11\left(\mathrm{~d}, J_{\mathrm{H}, \mathrm{H}}=6 \mathrm{~Hz}, 2 \mathrm{H} ; \mathrm{Ar}-\mathrm{H}\right)$, $5.03\left(\mathrm{~d}, J_{\mathrm{H}, \mathrm{H}}=6 \mathrm{~Hz}, 2 \mathrm{H} ; \mathrm{Ar}-\mathrm{H}\right), 4.66\left(\mathrm{~d}, J_{\mathrm{H}, \mathrm{H}}=6 \mathrm{~Hz}, 2 \mathrm{H} ; \mathrm{Ar}-\mathrm{H}\right), 2.52\left(\mathrm{spt}, J_{\mathrm{H}, \mathrm{H}}=6 \mathrm{~Hz}, 2 \mathrm{H} ; \mathrm{CH}\right), 2.00\left(\mathrm{~s}, 6 \mathrm{H} ; \mathrm{CH}_{3}\right), 1.11 \mathrm{ppm}(\mathrm{dd}$, $\left.J_{\mathrm{H}, \mathrm{H}}=12 \mathrm{~Hz}, 9 \mathrm{~Hz}, 12 \mathrm{H} ; \mathrm{CH}_{3}\right) .{ }^{19} \mathrm{~F} \mathrm{NMR}\left(282 \mathrm{MHz}, \mathrm{CDCl}_{3}\right): \delta=-61.98 \mathrm{ppm}\left(\mathrm{s}, \mathrm{CF}_{3}\right)$. IR (neat): $\tilde{v}=3061$ (w, aromatic C-H), 2927 (m, aliphatic C-H), 1573 (vs, oxamide C=O), 1317 (vs, $\mathrm{CF}_{3}$ ), 1102 (vs, C-N) cm ${ }^{-1}$.

\section{General Self-Assembly Procedure}

Briefly, the formation the following cofacial porphyrin prisms were afforded by the combination of the requisite building blocks with careful control over stoichiometry. In general, the chloro-ligated ruthenium clip (4 eq.) was treated with a slight excess of AgOTf (8.8 eq.) in a salt metathesis reaction in methanol (10 mL) forming either the solvato- or triflate-complex in situ. The salt metathesis reaction was performed with exclusion of light. Upon addition of AgOTf to the reaction mixture, the solution transitioned form an orange suspension to a light orange solution with a white precipitate, indicating the formation of $\mathrm{AgCl}(\mathrm{s})$. The reaction was stirred for 3 hours, after which the reaction was filtered through a fiber glass filter removed the $\mathrm{AgCl}$ precipitate. The filtrate was added directly to a suspension of the metallated TPyP (2 eq., either ZnTPyP or CoTPyP) in $5 \mathrm{~mL}$ of methanol. The reaction was then brought to reflux for 48 hours when ZnTPyP was used, and 96 hours when CoTPyP was used. This disparity in reaction time arises due to the stark differences in solubility between ZnTPyP and CoTPyP, with CoTPyP showing far lower solubility. Nevertheless, at the completion of reaction, the reaction mixture was a deep green (when ZnTPyP was used) or red (when CoTPyP was used) homogeneous solution. The solvent was then removed by rotary evaporation, and redissolved in the minimal amount of methanol. The solution was then filtered directly into diethyl ether to precipitate the desired cofacial porphyrin prism. The precipitate was collected by centrifugation. The supernatant was discarded and the solid dried in vacuo for at least 12 hours before spectroscopic or electrochemical characterization. 


\section{Zn-Tolyl Prism Synthesis}

$20.0 \mathrm{mg}$ (0.0248 mmol, 4.00 eq.) Ru-Tolyl clip, $13.9 \mathrm{mg}$ (0.0545 mmol, 8.80 eq.) AgOTf, $8.40 \mathrm{mg}(0.0123 \mathrm{mmol}, 2 \mathrm{eq}$. ZnTPyP, in $15 \mathrm{~mL}$ methanol. Yield: $29.9 \mathrm{mg}(0.00543 \mathrm{mmol})$ Zn-Tolyl Prism, 87.2\%. ${ }^{1} \mathrm{H}$ NMR (500 MHz, CD $\left.{ }_{3} \mathrm{OD}\right): \delta=9.34-$ 8.35 ( $m, 43 \mathrm{H}$; pyrrole, pyridyl, phenyl Ar-H), 8.16 ( $s, 2 \mathrm{H}$; pyrrole, pyridyl, phenyl Ar-H), 8.06-7.78 ( $m$, 10H; pyrrole, pyridyl, phenyl Ar-H), 7.78-7.57 ( $m, 16 \mathrm{H}$; pyrrole, pyridyl, phenyl Ar-H), 7.48 ( $d, J=10,9 \mathrm{H}$; pyrrole, pyridyl, phenyl Ar-H), 6.535.23 (m, 32H; p-Cymene Ar-H), 3.03-2.75 (m, 8H; isopropyl CH), 2.69-2.31 (m, 24H; Tolyl CH $), 2.25-1.65$ (m, $24 \mathrm{H}$; pCymene $\mathrm{CH}_{3}$ ), 1.62-1.23 (m, 48H; isopropyl $\mathrm{CH}_{3}$ ) FT-IR $\left(v, \mathrm{~cm}^{-1}\right)$ : 3070, 2926, 1580, 1219, 1256, 1150, 1022, 991, 799, 635, 571, 513. UV-VIS (MeOH): Soret band: $\lambda \max =423 \mathrm{~nm}, \varepsilon=1.1 \times 10^{6} \mathrm{M}^{-1} \mathrm{~cm}^{-1} \mathrm{Q}$-band $1: \lambda_{\max }=569 \mathrm{~nm}, \varepsilon=4.1 \times 10^{4} \mathrm{M}^{-1} \mathrm{~cm}^{-1}$ Q-band 2: $\lambda_{\max }=615 \mathrm{~nm}, \varepsilon=2.4 \times 10^{4} \mathrm{M}^{-1} \mathrm{~cm}^{-1}$.

\section{Co-Tolyl Prism Synthesis}

$20.0 \mathrm{mg}$ (0.0248 mmol, 4.00 eq.) Ru-Tolyl clip, $13.9 \mathrm{mg}$ (0.0545 mmol, 8.80 eq.) AgOTf, $8.45 \mathrm{mg}$ (0.0123 mmol, 2 eq.) CoTPyP, in $15 \mathrm{~mL}$ methanol. Yield: $28.3 \mathrm{mg}$ (0.00505 mmol) Co-Tolyl prism, 82.1\%. FT-IR (v, $\left.\mathrm{cm}^{-1}\right): 3070,2926,1578$, $1256,1219,1148,1027,999,799,635,570,519$. UV-VIS $(\mathrm{MeOH})$ : Soret band: $\lambda \mathrm{max}=417 \mathrm{~nm}, \varepsilon=3.1 \times 10^{5} \mathrm{M}^{-1} \mathrm{~cm}^{-1} \mathrm{Q}$ band 1: $\lambda_{\max }=545 \mathrm{~nm}, \varepsilon=2.4 \times 10^{4} \mathrm{M}^{-1} \mathrm{~cm}^{-1} \mathrm{Q}$ band $2: \lambda_{\max }=581 \mathrm{~nm}, \varepsilon=1.2 \times 10^{4} \mathrm{M}^{-1} \mathrm{~cm}^{-1}$.

\section{Zn-Phenyl Prism Synthesis}

$20.0 \mathrm{mg}$ (0.0256 mmol, 4.00 eq.) Ru-Phenyl clip, $14.8 \mathrm{mg}$ (0.0576 mmol, 8.80 eq.) AgOTf, $8.78 \mathrm{mg}$ (0.0128 mmol, 2.00 eq.) ZnTPyP, in $15 \mathrm{~mL}$ methanol. Yield: $33.0 \mathrm{mg}$ (0.00612 mmol) Zn-Phenyl prism, 94.5\%. ${ }^{1} \mathrm{H} \mathrm{NMR}\left(500 \mathrm{MHz}, \mathrm{CD}_{3} \mathrm{OD}\right): \delta=9.32-$ 8.76 ( $m, 22 \mathrm{H}$; pyrrole, pyridyl, phenyl Ar-H), 8.70-8.33 ( $m, 14 \mathrm{H}$; pyrrole, pyridyl, phenyl Ar-H), 8.24-8.04 ( $m$, 8H; pyrrole, pyridyl, phenyl Ar-H), 8.01-7.75 ( $m, 22 \mathrm{H}$; pyrrole, pyridyl, phenyl Ar-H), 7.74-7.33 ( $m$, 22H; pyrrole, pyridyl, phenyl Ar-H), 6.61-5.00 (m, 32H; p-Cymene Ar-H), 3.00-2.75 (m, 8H; isopropyl CH), 2.59-1.71 (m, 24H; p-Cymene $\left.\mathrm{CH}_{3}\right), 1.58-1.26(\mathrm{~m}$, 48H; isopropyl $\left.\mathrm{CH}_{3}\right)$. FT-IR $\left(v, \mathrm{~cm}^{-1}\right)$ : 3070, 2971, 1737, 1563, 1488, 1253, 1228, 1155 1027, 999, 792, 630, 569, 513. UVVIS $(\mathrm{MeOH})$ : Soret band: $\lambda \max =423 \mathrm{~nm}, \varepsilon=9.9 \times 10^{5} \mathrm{M}^{-1} \mathrm{~cm}^{-1} \mathrm{Q}$ band $1: \lambda_{\max }=570 \mathrm{~nm}, \varepsilon=4.1 \times 10^{4} \mathrm{M}^{-1} \mathrm{~cm}^{-1} \mathrm{Q} \mathrm{band}_{2}$ : $\lambda_{\max }=613 \mathrm{~nm}, \varepsilon=2.4 \times 10^{4} \mathrm{M}^{-1} \mathrm{~cm}^{-1}$.

\section{Co-Phenyl Prism Synthesis}

$20.0 \mathrm{mg}$ (0.0256 mmol, 4.00 eq.) Ru-Phenyl clip, $14.8 \mathrm{mg}$ (0.0576 mmol, 8.80 eq.) AgOTf, $8.67 \mathrm{mg}(0.0128 \mathrm{mmol}, 2.00$ eq.) CoTPyP, in $15 \mathrm{~mL}$ methanol. Yield: $34.4 \mathrm{mg}(0.00627 \mathrm{mmol})$ Co-Phenyl prism, 98.2\%. FT-IR (v, $\left.\mathrm{cm}^{-1}\right): 3070,2971,1741$, 1565, 1486, 1253, 1224, 1155 1027, 999, 792, 635, 566, 508. UV-VIS (MeOH): Soret band: $\lambda \max =415 \mathrm{~nm}, \varepsilon=3.7 \times 10^{5} \mathrm{M}$ ${ }^{1} \mathrm{~cm}^{-1} \mathrm{Q}$ band 1: $\lambda_{\max }=544 \mathrm{~nm}, \varepsilon=3.6 \times 10^{4} \mathrm{M}^{-1} \mathrm{~cm}^{-1} \mathrm{Q}$ band $2: \lambda_{\max }=584 \mathrm{~nm}, \varepsilon=1.9 \times 10^{4} \mathrm{M}^{-1} \mathrm{~cm}^{-1}$.

\section{Zn-Chloro Prism Synthesis}

$20.0 \mathrm{mg}$ (0.0236 mmol, 4.00 eq.) Ru-Chloro clip, $13.4 \mathrm{mg}$ (0.0519 mmol, 8.80 eq.) AgOTf, $8.05 \mathrm{mg}$ (0.0118 mmol, 2.00 eq.) ZnTPyP, in $15 \mathrm{~mL}$ methanol. Yield: $33.0 \mathrm{mg}(0.00502 \mathrm{mmol})$ Zn-Chloro prism, 85.0\%. ${ }^{1} \mathrm{H} \mathrm{NMR}\left(500 \mathrm{MHz}, \mathrm{CD}{ }_{3} \mathrm{OD}\right)$ : $\delta=9.34-9.20$ ( $m$, 5H; pyrrole, pyridyl, phenyl Ar-H), 9.15-8.96 ( $m, 13 \mathrm{H}$; pyrrole, pyridyl, phenyl Ar-H), 8.94-8.77 $(m, 13 \mathrm{H}$; pyrrole, pyridyl, phenyl Ar-H), 8.74-8.38 ( $m, 15 \mathrm{H}$; pyrrole, pyridyl, phenyl Ar-H), 8.25-8.11 ( $m$, 6H; pyrrole, pyridyl, phenyl Ar-H), 7.98-7.80 ( $m, 19 \mathrm{H}$; pyrrole, pyridyl, phenyl Ar-H), 7.69-7.54 ( $\mathrm{m}, 9 \mathrm{H}$; pyrrole, pyridyl, phenyl Ar-H), 6.45-5.31 (m, 32H; p-Cymene Ar-H), 3.09-2.77 (m, 8H; isopropyl CH), 2.25-1.72 (m, 24H; p-Cymene $\left.\mathrm{CH}_{3}\right), 1.71-1.24$ (m, $48 \mathrm{H}$; isopropyl $\left.\mathrm{CH}_{3}\right)$. FT-IR $\left(v, \mathrm{~cm}^{-1}\right)$ : 3070, 2971, 1742, 1575, 1486, 1251, 1234, 1027, 999, 792, 635, 562, 516. UV-VIS (MeOH): Soret band: $\lambda \max =423 \mathrm{~nm}, \varepsilon=7.8 \times 10^{5} \mathrm{M}^{-1} \mathrm{~cm}^{-1} \mathrm{Q}$ band $1: \lambda_{\max }=569 \mathrm{~nm}, \varepsilon=2.1 \times 10^{4} \mathrm{M}^{-1} \mathrm{~cm}^{-1} \mathrm{Q}$ band $2: \lambda_{\max }=614 \mathrm{~nm}, \varepsilon=$ $6.6 \times 10^{3} \mathrm{M}^{-1} \mathrm{~cm}^{-1}$.

\section{Co-Chloro Prism Synthesis}

$20.0 \mathrm{mg}$ (0.0236 mmol, 4.00 eq.) Ru-Chloro clip, $13.4 \mathrm{mg}$ (0.0519 mmol, 8.80 eq.) AgOTf, $8.01 \mathrm{mg}(0.0118 \mathrm{mmol}, 2.00 \mathrm{eq}$. CoTPyP, in $15 \mathrm{~mL}$ methanol. Yield: $33.0 \mathrm{mg}(0.00480 \mathrm{mmol})$ Co-Chloro prism, 81.3\%. FT-IR ( $v$, $\left.\mathrm{cm}^{-1}\right): 3070,2971,1741$, $1578,1480,1253,1234,1027,999,792,635,562,513$. Soret band: $\lambda \max =417 \mathrm{~nm}, \varepsilon=2.1 \times 10^{5} \mathrm{M}^{-1} \mathrm{~cm}^{-1} \mathrm{Q}{\text { band } 1: \lambda_{\max }=}$ $545 \mathrm{~nm}, \varepsilon=1.2 \times 10^{4} \mathrm{M}^{-1} \mathrm{~cm}^{-1} \mathrm{Q}$ band $2: \lambda_{\max }=578 \mathrm{~nm}, \varepsilon=2.5 \times 10^{3} \mathrm{M}^{-1} \mathrm{~cm}^{-1}$. 


\section{Zn-CF 3 Prism Synthesis}

$20.0 \mathrm{mg}$ (0.0218 mmol, 4.00 eq.) Ru-CF3 clip, $12.3 \mathrm{mg}$ (0.0481 mmol, 8.80 eq.) AgOTf, $7.43 \mathrm{mg}$ (0.0109 mmol, 2.00 eq.) ZnTPyP, in $15 \mathrm{~mL}$ methanol. Yield: $31.56 \mathrm{mg}$ (0.00531 mmol) $\mathbf{Z n - C F} 3$ prism, 96.6\%. ${ }^{1} \mathrm{H}$ NMR (500 MHz, CD $\left.{ }_{3} \mathrm{OD}\right): \delta=9.51-$ 7.44 ( $m, 80 \mathrm{H}$; pyrrole, Pyridyl, Phenyl Ar-H), 6.45-5.41 (m, 32H; p-Cymene Ar-H), 3.05-2.82 (m, 8H; isopropyl CH), 2.191.79 (m, 24H; p-Cymene $\left.\mathrm{CH}_{3}\right), 1.62-1.15\left(\mathrm{~m}, 48 \mathrm{H}\right.$; isopropyl $\left.\mathrm{CH}_{3}\right) .{ }^{19} \mathrm{~F} \mathrm{NMR}\left(282 \mathrm{MHz}, \mathrm{CD}_{3} \mathrm{OD}\right): \delta=-63.70\left(\mathrm{~s}, \mathrm{CF}_{3}\right),-79.22$ (s, OTf $\left.{ }^{-}\right)$. FT-IR $\left(v, \mathrm{~cm}^{-1}\right): 3070,2970,1573,1316,1257,1157,1027,1005,797,629,571,515$. UV-VIS (MeOH): Soret band: $\lambda \max =425 \mathrm{~nm}, \varepsilon=2.2 \times 10^{5} \mathrm{M}^{-1} \mathrm{~cm}^{-1} \mathrm{Q}$ band $1: \lambda_{\max }=573 \mathrm{~nm}, \varepsilon=1.5 \times 10^{4} \mathrm{M}^{-1} \mathrm{~cm}^{-1} \mathrm{Q}$ band $2: \lambda_{\max }=616 \mathrm{~nm}, \varepsilon=$ $1.1 \times 10^{4} \mathrm{M}^{-1} \mathrm{~cm}^{-1}$.

\section{Co-CF 3 Prism Synthesis}

$20.0 \mathrm{mg}$ (0.0218 mmol, 4.00 eq.) Ru-CF 3 clip, $12.3 \mathrm{mg}$ (0.0481 mmol, 8.80 eq.) AgOTf, $7.41 \mathrm{mg}$ (0.0109 mmol, 2.00 eq.) CoTPyP, in $15 \mathrm{~mL}$ methanol. Yield: $30.9 \mathrm{mg}$ (0.00516 mmol) Co-CF3 prism, 94.6\%. FT-IR (v, $\left.\mathrm{cm}^{-1}\right): 3070,2970,1739,1574$, $1255,1157,1027,1005,800,635,571,519$. UV-VIS $(\mathrm{MeOH})$ : Soret band: $\lambda \max =423 \mathrm{~nm}, \varepsilon=3.1 \times 10^{5} \mathrm{M}^{-1} \mathrm{~cm}^{-1} \mathrm{Q} \mathrm{band}_{1}$ : $\lambda_{\max }=547 \mathrm{~nm}, \varepsilon=3.8 \times 10^{4} \mathrm{M}^{-1} \mathrm{~cm}^{-1} \mathrm{Q}$ band $2: \lambda_{\max }=583 \mathrm{~nm}, \varepsilon=2.3 \times 10^{4} \mathrm{M}^{-1} \mathrm{~cm}^{-1}$.

\section{Results and Discussion}

\section{Spectroscopic Characterization}

\section{Mass Spectrometry:}

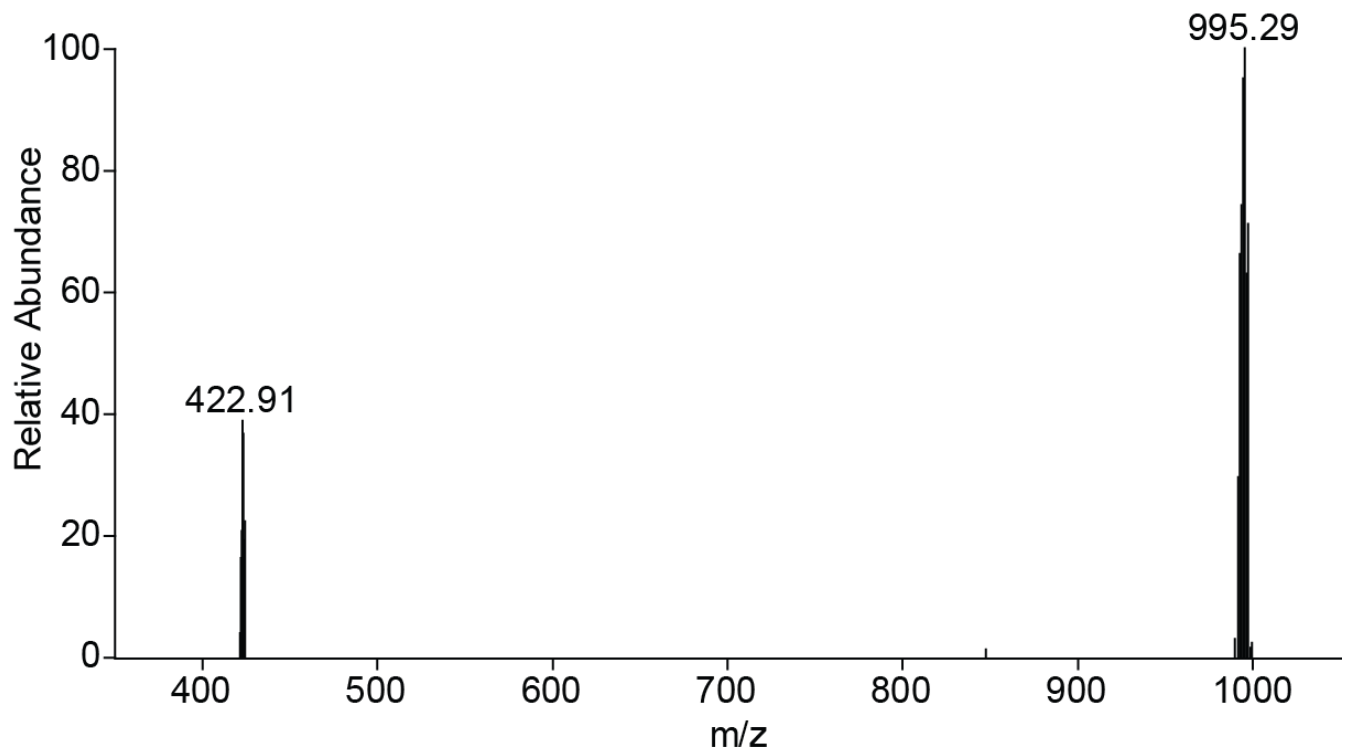

Figure S1. Mass spectrum of Ru-CF 3 . To improve solubility and enhance ionization, 2 eq. of AgOTf were added and the sample filtered before injection (Thermo LTQ). The peak at 995.29 corresponds to the $\left[\mathbf{R u}-\mathbf{C F}_{3}-2 \mathbf{C l}^{2-}+\mathrm{OTf}^{-}\right]^{+}$and 422.91 to $\left[\mathbf{R u}-\mathbf{C F}_{3}-2 \mathrm{Cl}^{2-}\right]^{2+}$. 


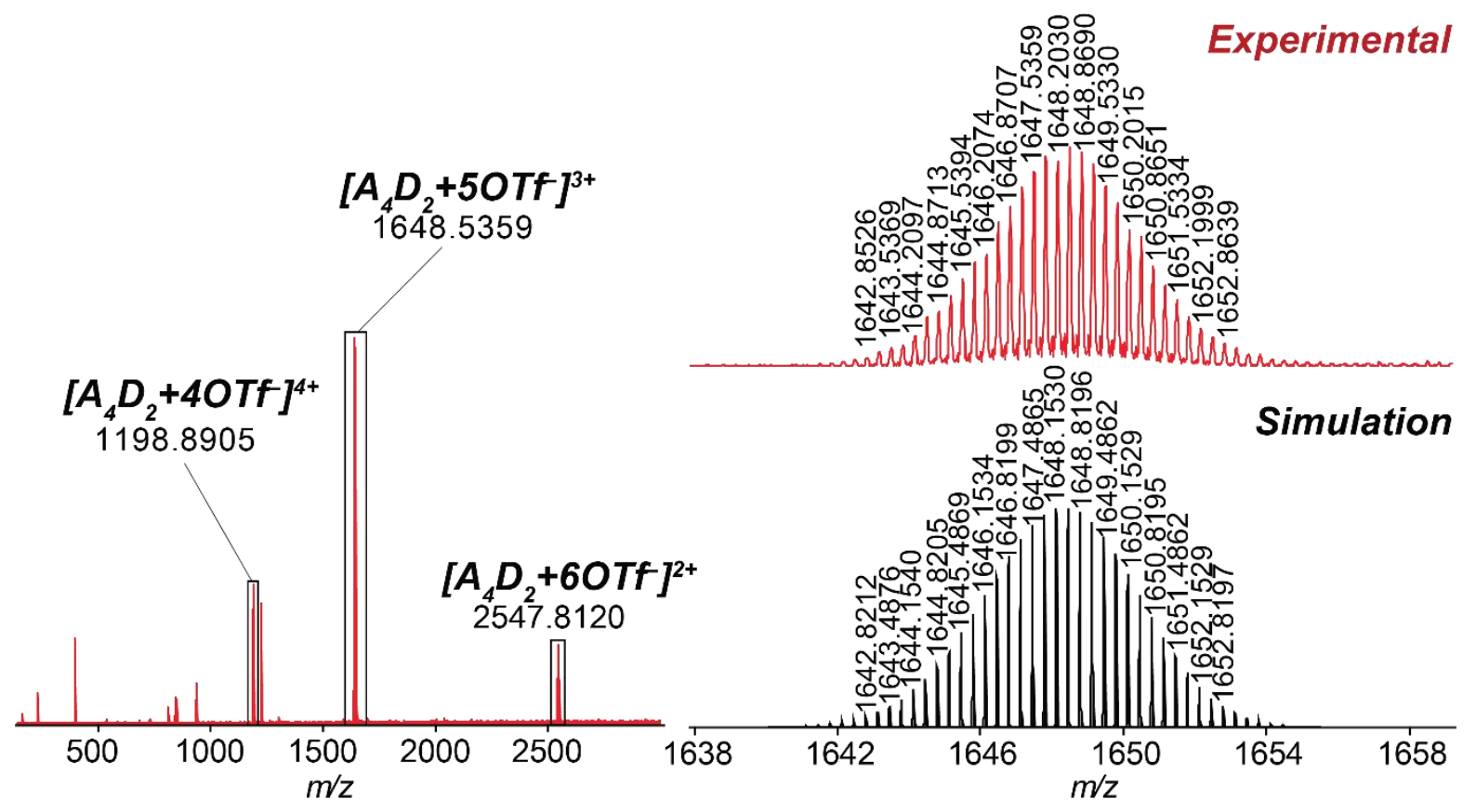

Figure S2. ESI-FT-ICR mass spectrum of Zn-Phenyl Prism. (left) Full spectrum, (right) Zoom-in on intact prism 3+ peak.

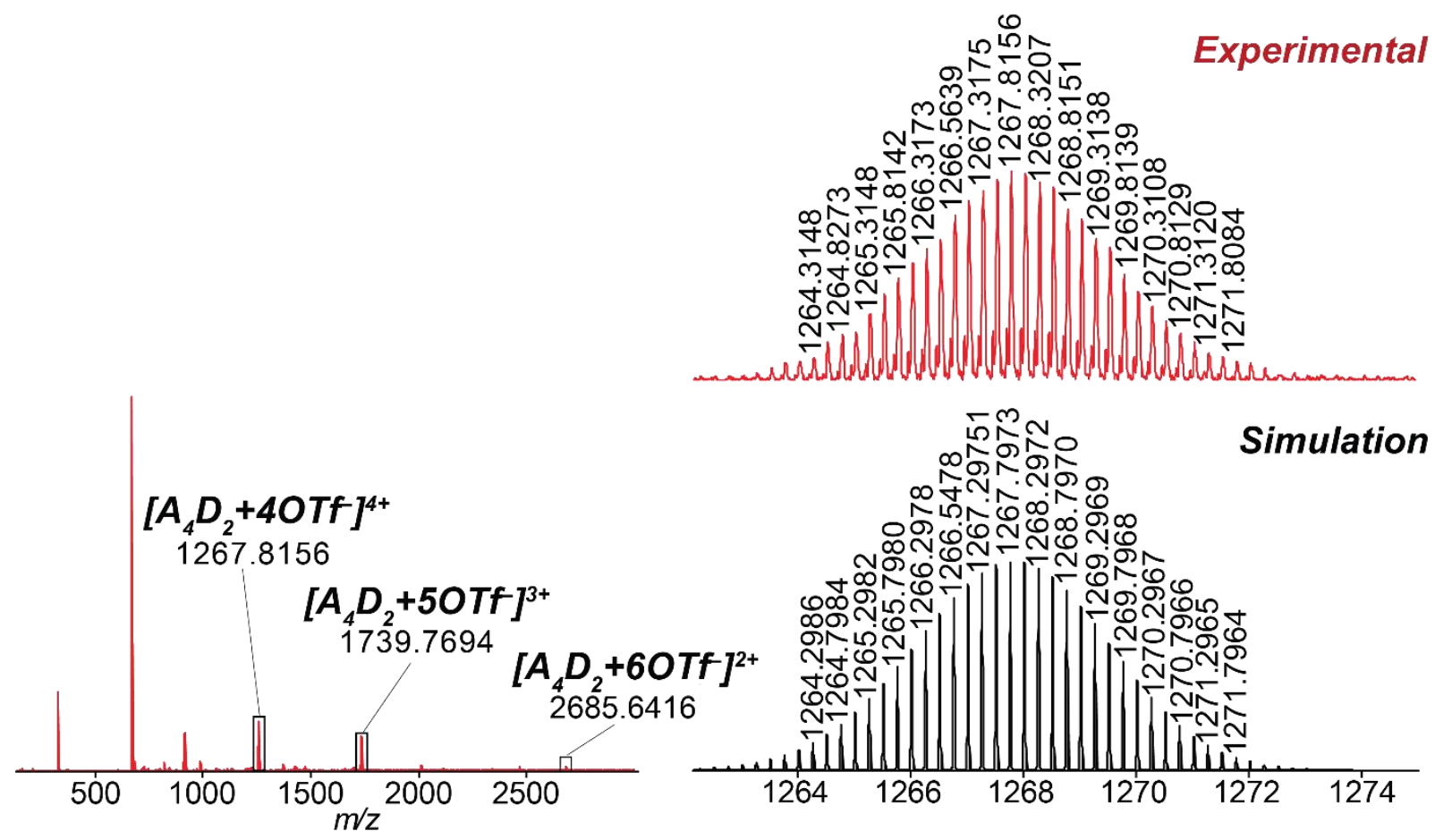

Figure S3. ESI-FT-ICR mass spectrum of Zn-Chloro Prism. (left) Full spectrum, (right) Zoom-in on intact prism 4+ peak. 


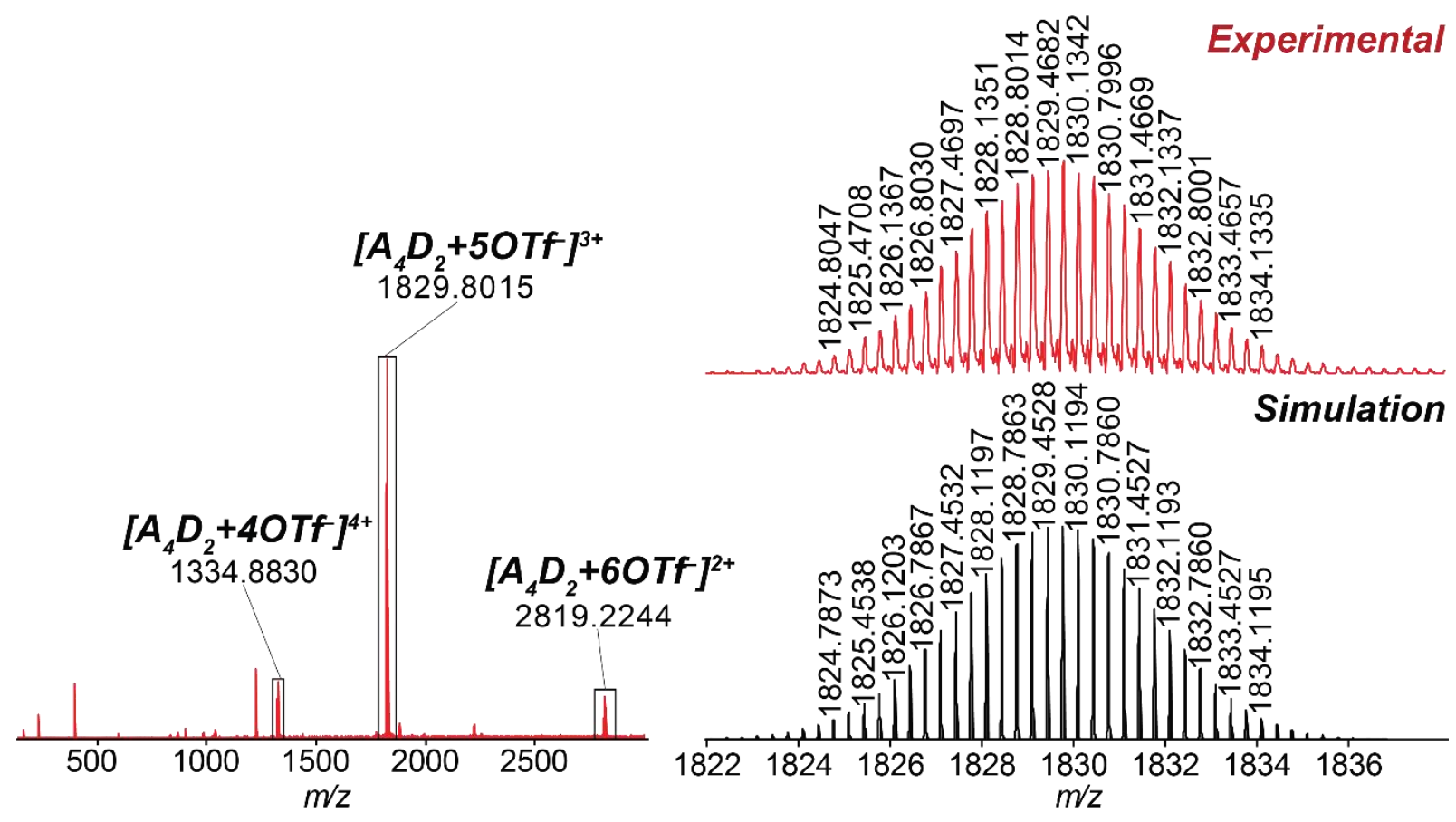

Figure S4. ESI-FT-ICR mass spectrum of Zn-CF3 Prism. (left) Full spectrum, (right) Zoom-in on intact prism 3+ peak.

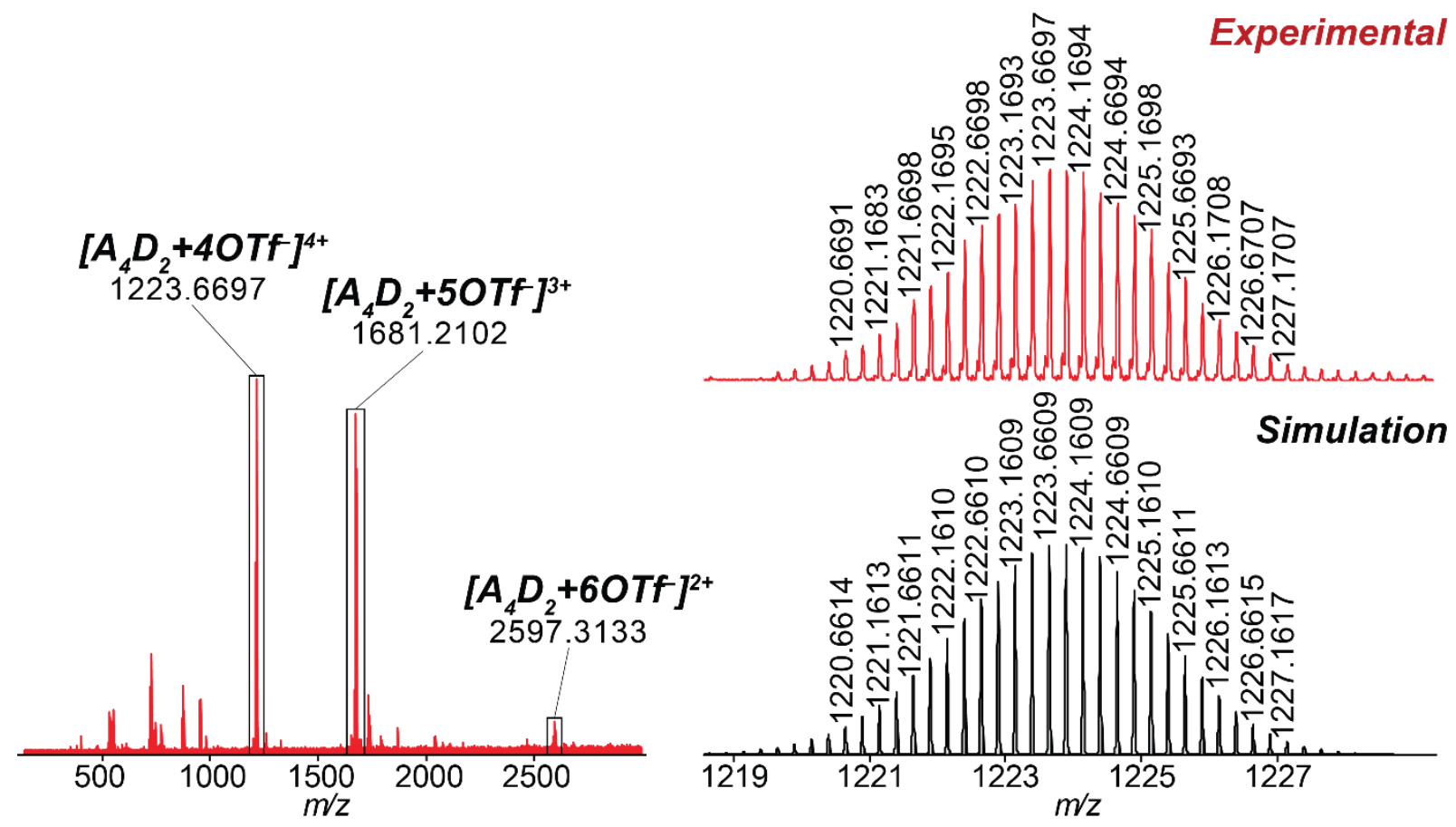

Figure S5. ESI-FT-ICR mass spectrum of Co-Tolyl Prism. (left) Full spectrum, (right) Zoom-in on intact prism 4+ peak. 


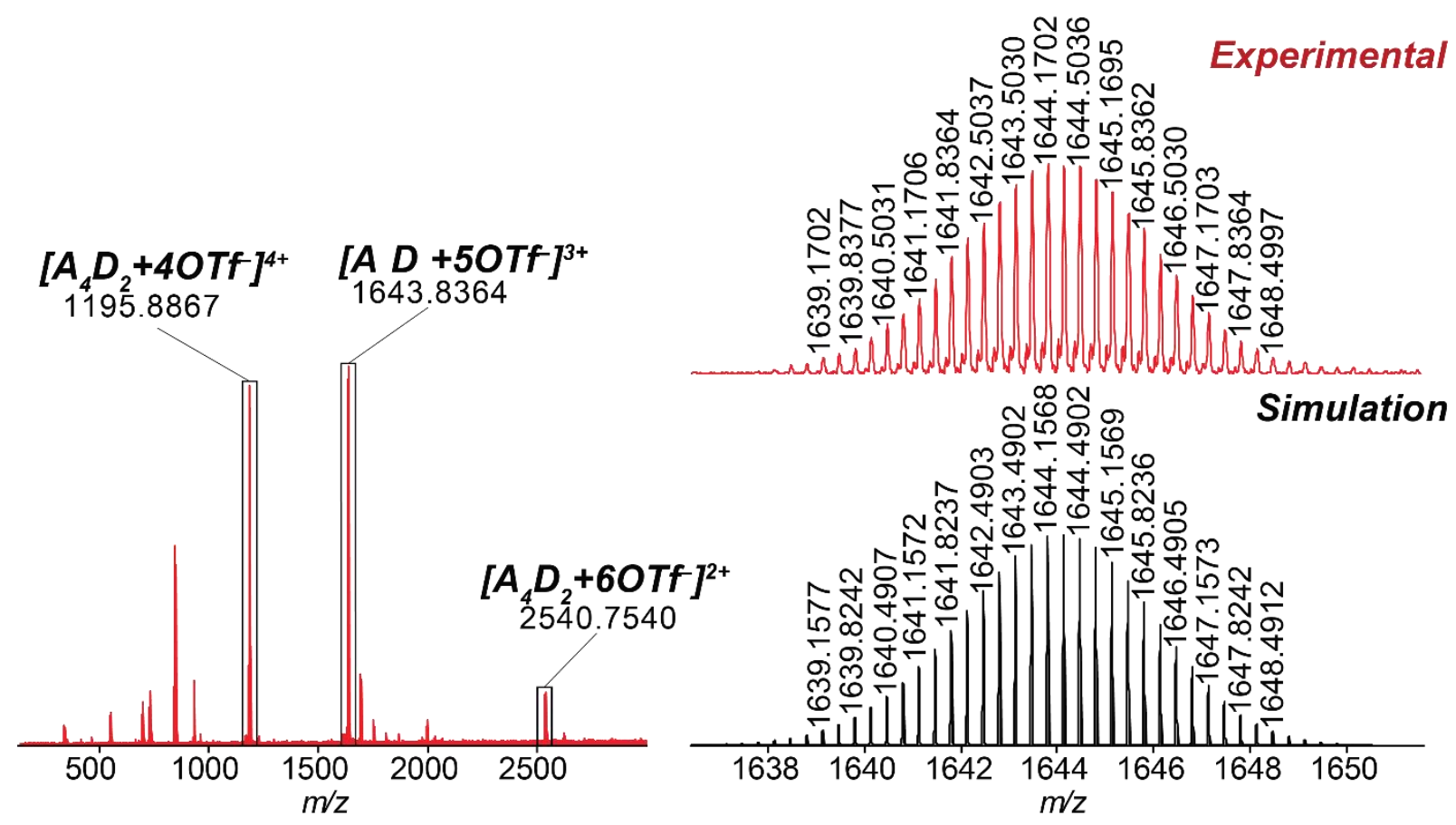

Figure S6. ESI-FT-ICR mass spectrum of Co-Phenyl Prism. (left) Full spectrum, (right) Zoom-in on intact prism 3+ peak.

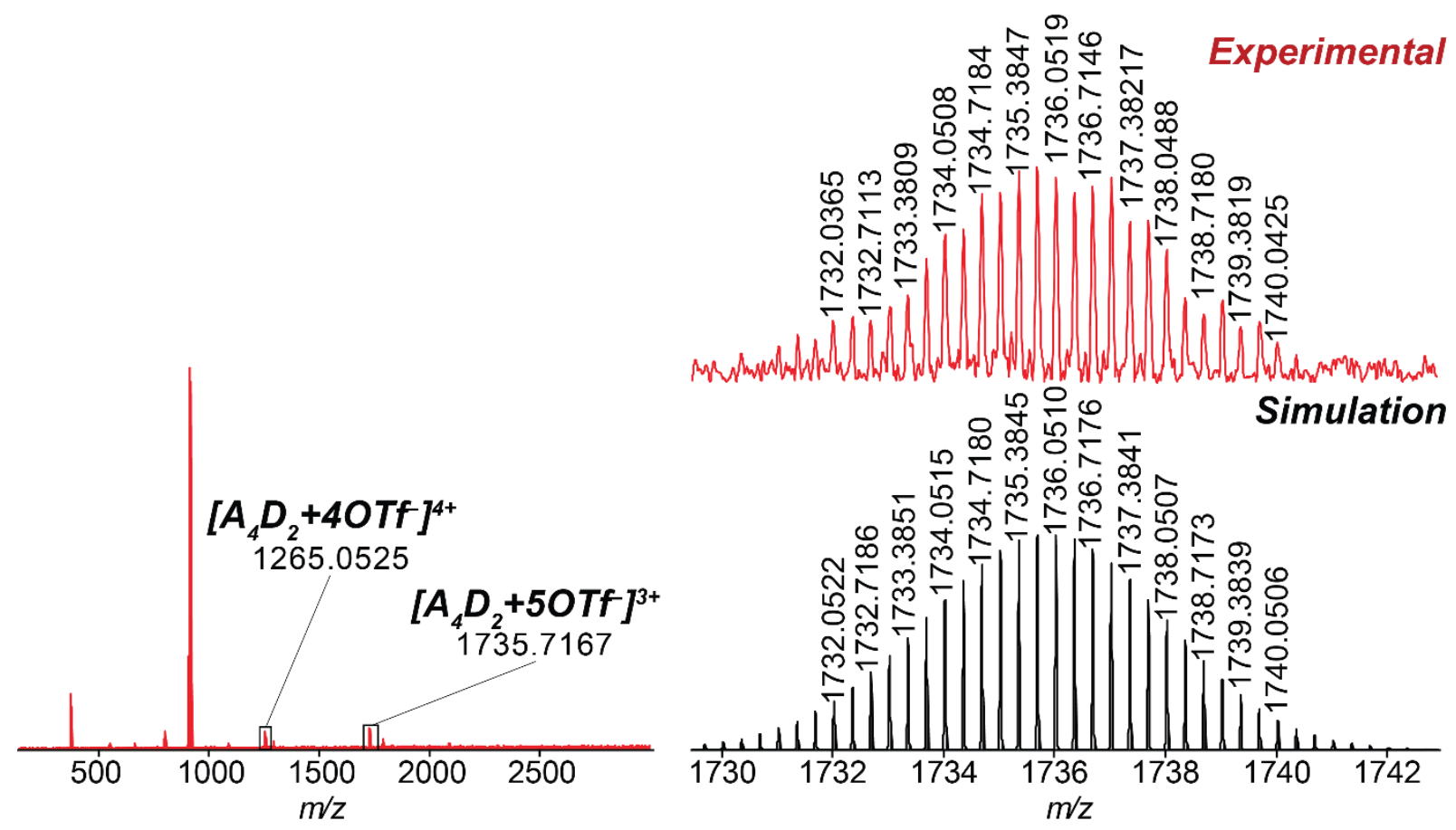

Figure S7. ESI-FT-ICR mass spectrum of Co-Chloro Prism. (left) Full spectrum, (right) Zoom-in on intact prism 3+ peak. 


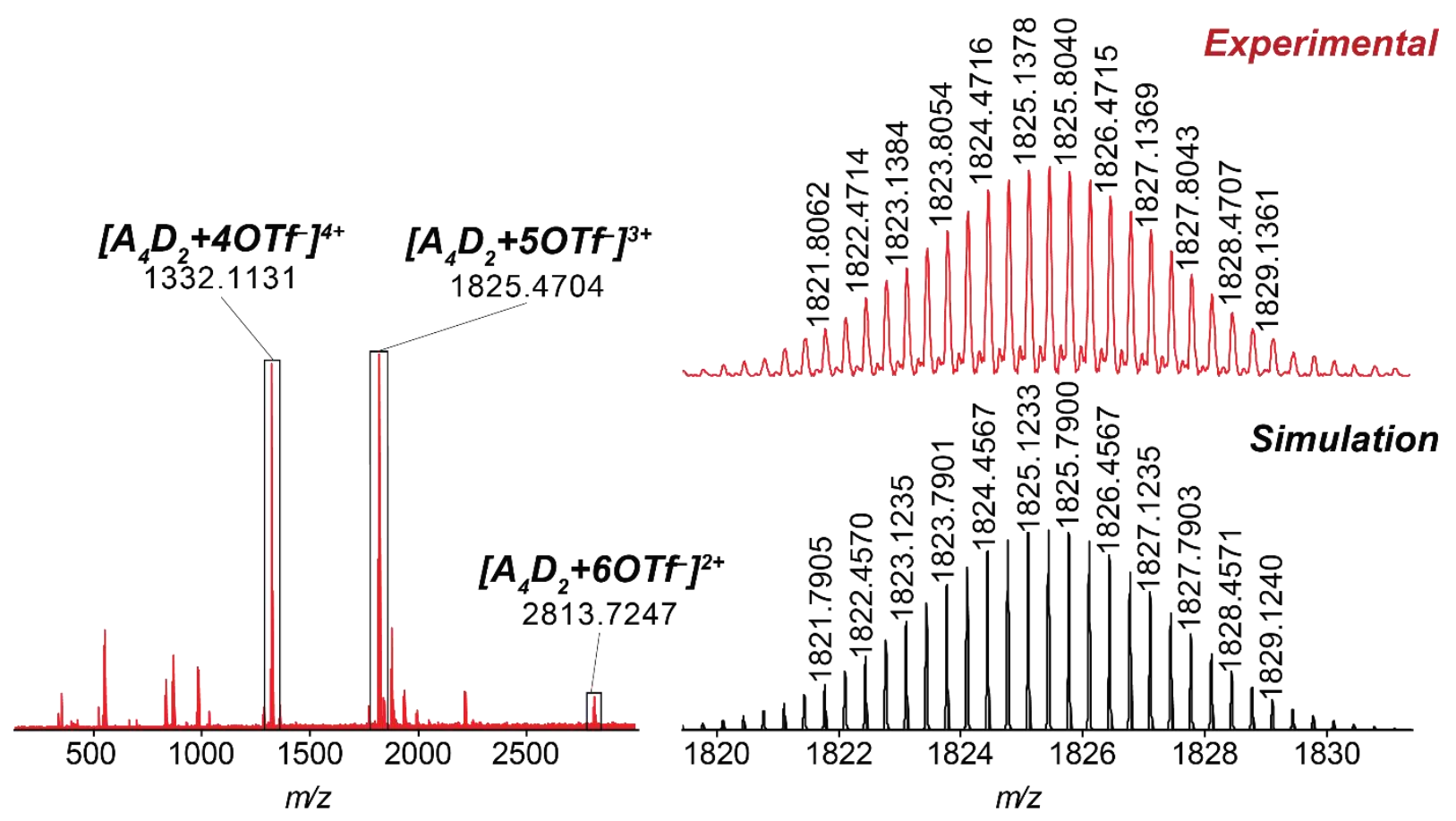

Figure S8. ESI-FT-ICR mass spectrum of Co-CF3 Prism. (left) Full spectrum, (right) Zoom-in on intact prism 3+ peak. 


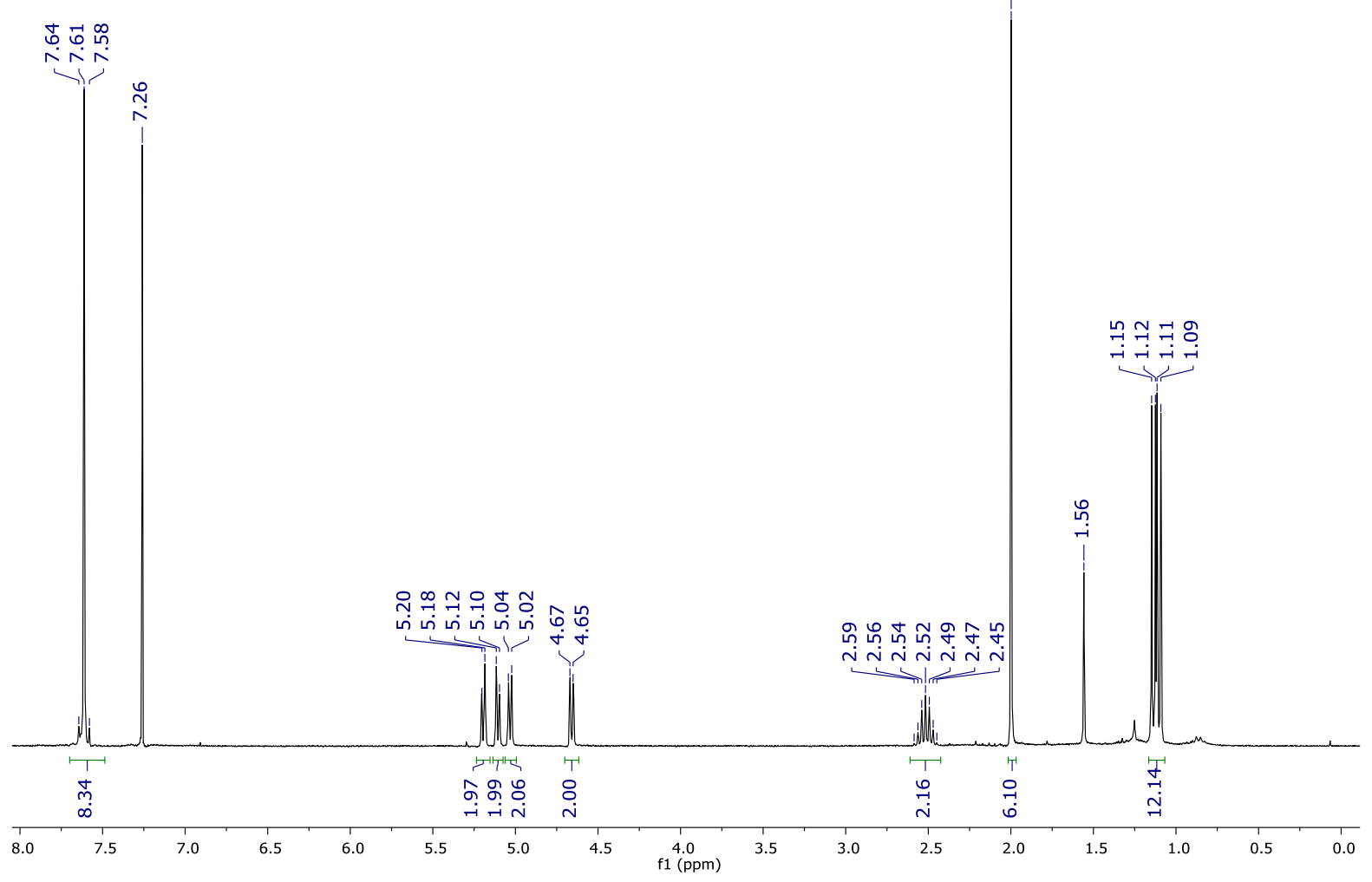

Figure S9. ${ }^{1} \mathrm{H}$ NMR of $\mathbf{R u - C F} 3$ Clip $\left(\mathrm{CDCl}_{3}, 300 \mathrm{MHz}\right)$. Residual proteo solvent resonance was observed at $7.26 \mathrm{ppm}$ along with water at $1.56 \mathrm{ppm}$.

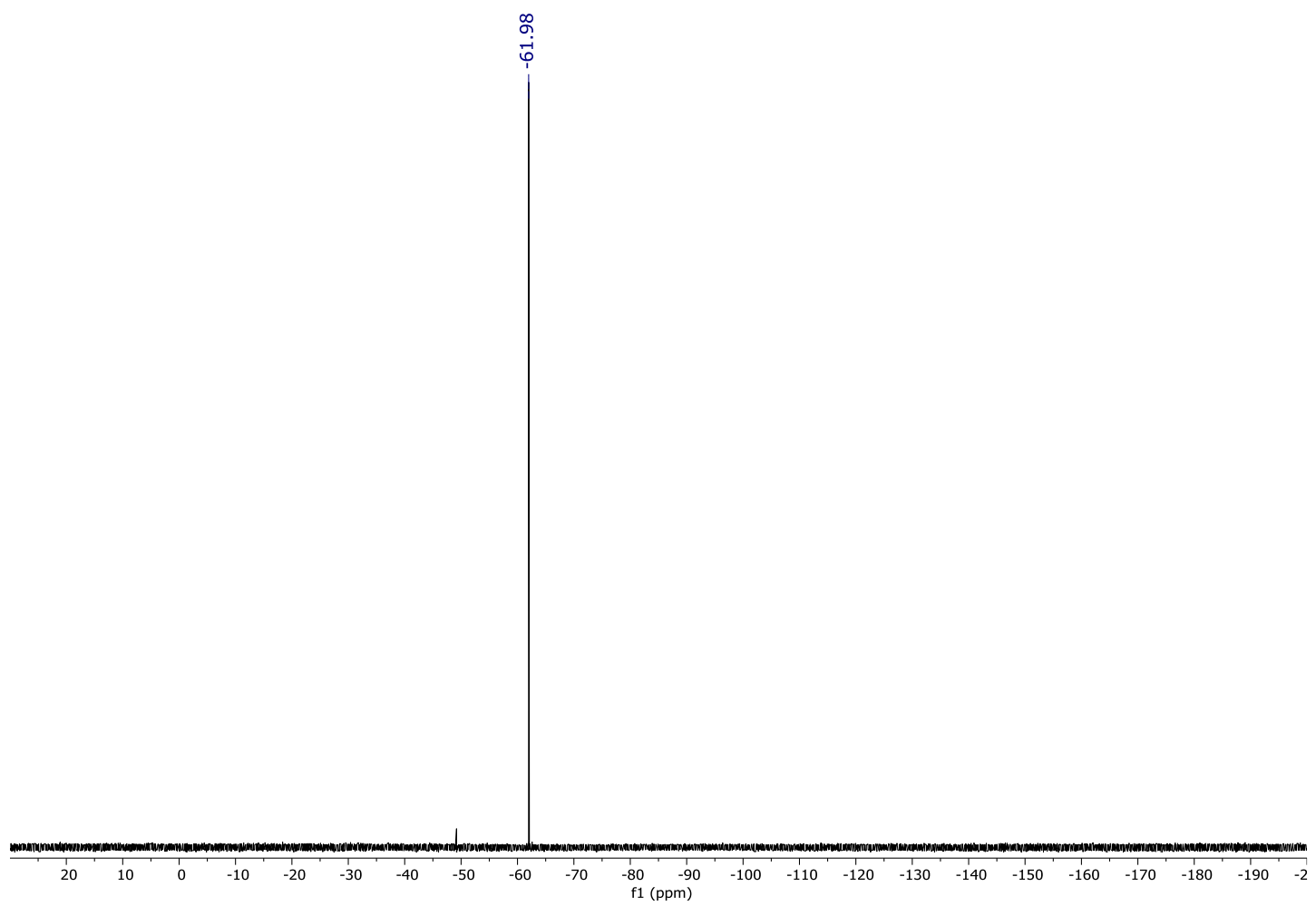

Figure S10. ${ }^{19} \mathrm{~F}\left\{{ }^{1} \mathrm{H}\right\}$ NMR of $\mathbf{R u}-\mathbf{C F}_{3}$ Clip $\left(\mathrm{CDCl}_{3}, 282 \mathrm{MHz}\right)$. Small resonance at $-48 \mathrm{ppm}$ is an instrumental artefact. 


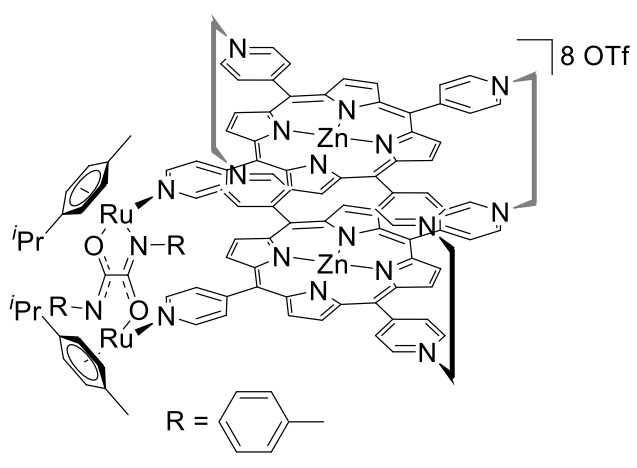

Aromatic

pyridyl, pyrrolic, and tolyl

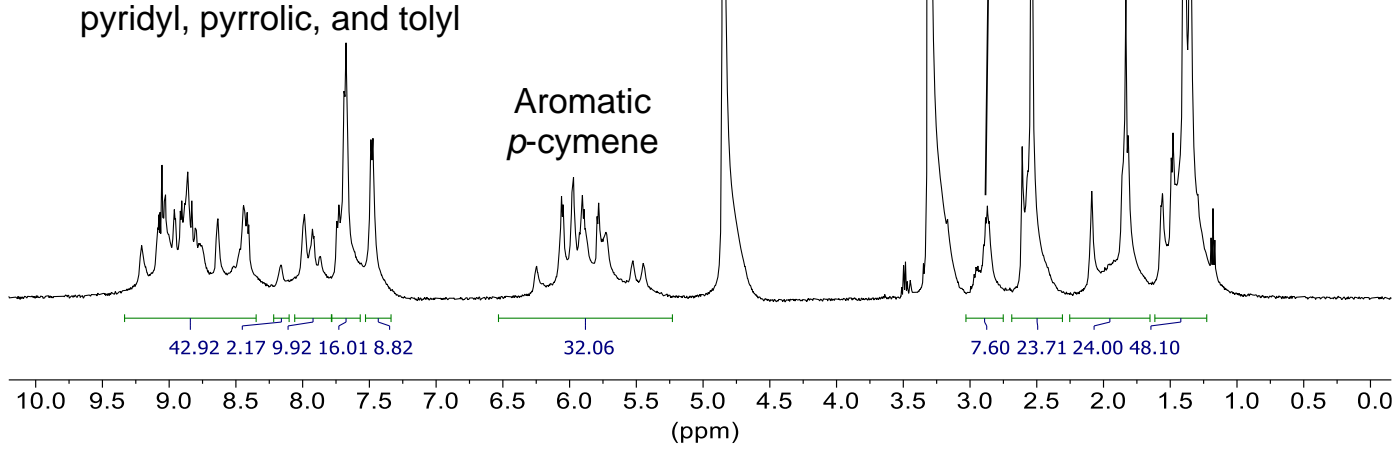

Figure S11. ${ }^{1} \mathrm{H}$ NMR of Zn-Tolyl Prism $\left(\mathrm{CD}_{3} \mathrm{OD}, 500 \mathrm{MHz}\right)$. Resonances at 4.85 and 3.31 ppm correspond to $\mathrm{H}_{2} \mathrm{O}$ and $\mathrm{CH}_{3} \mathrm{OH}$. Quartet at 3.49 and triplet at $1.18 \mathrm{ppm}$ correspond to diethyl ether used to precipitate. All other resonances can be attributed to the intact prism.

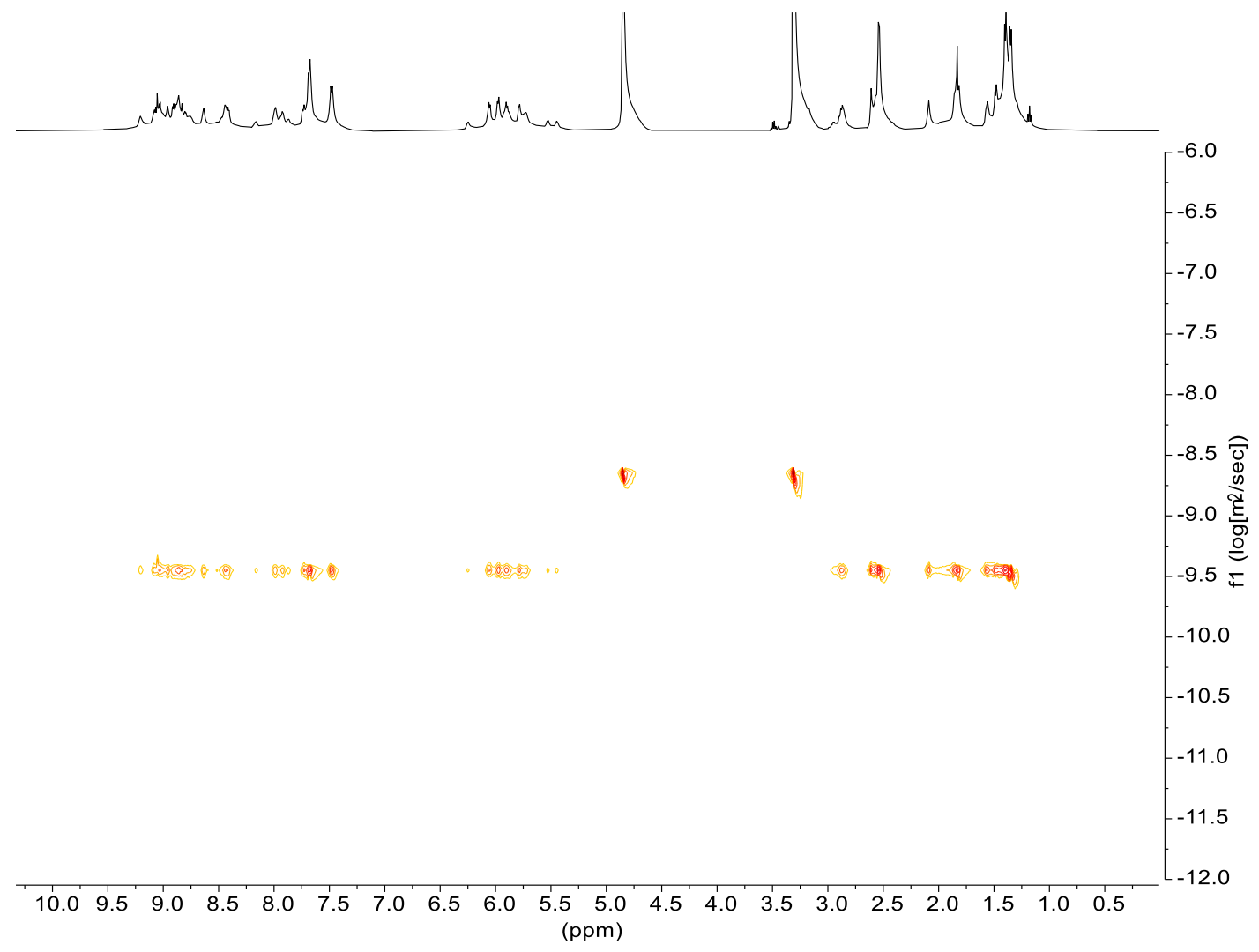

Figure S12. DOSY NMR spectrum of Zn-Tolyl Prism (CD $\left.{ }_{3} \mathrm{OD}, 500 \mathrm{MHz}\right)$. 


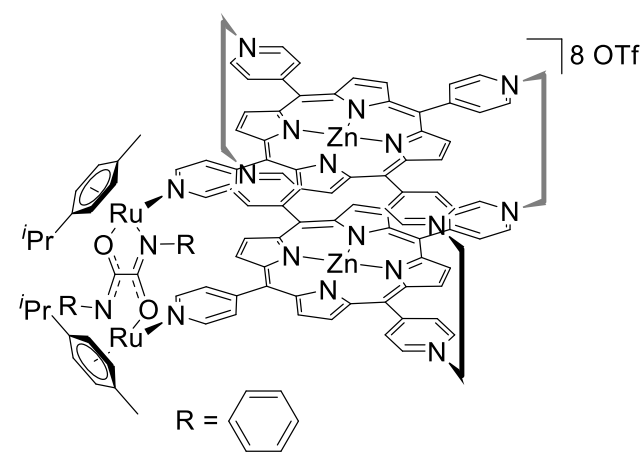

Aromatic

pyridyl, pyrrolic, and phenyl

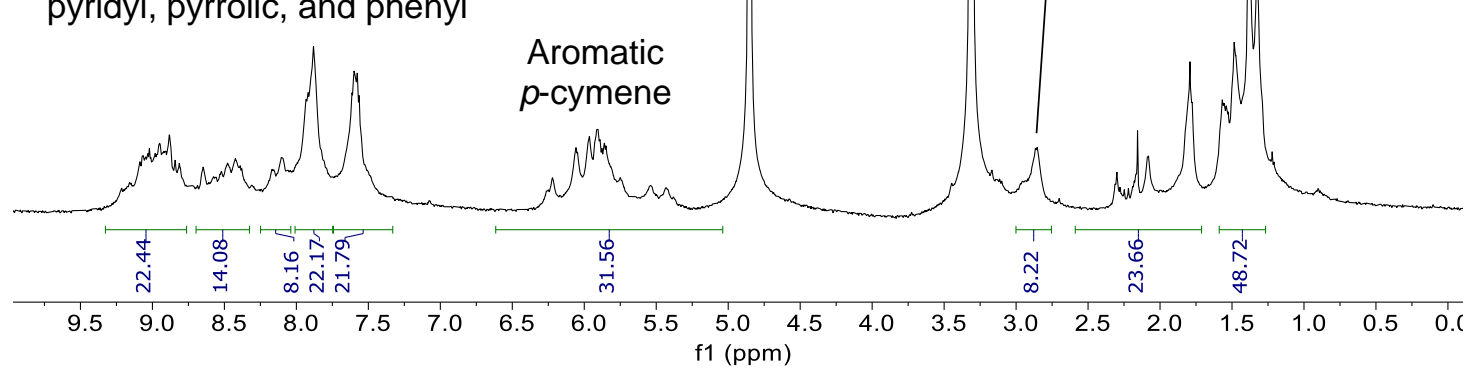

Figure S13. ${ }^{1} \mathrm{H}$ NMR of $\mathbf{Z n}$-Phenyl Prism $\left(\mathrm{CD}_{3} \mathrm{OD}, 500 \mathrm{MHz}\right)$. Resonances at 4.85 and $3.31 \mathrm{ppm}$ correspond to $\mathrm{H}_{2} \mathrm{O}$ and $\mathrm{CH}_{3} \mathrm{OH}$. All other resonances can be attributed to the intact prism.

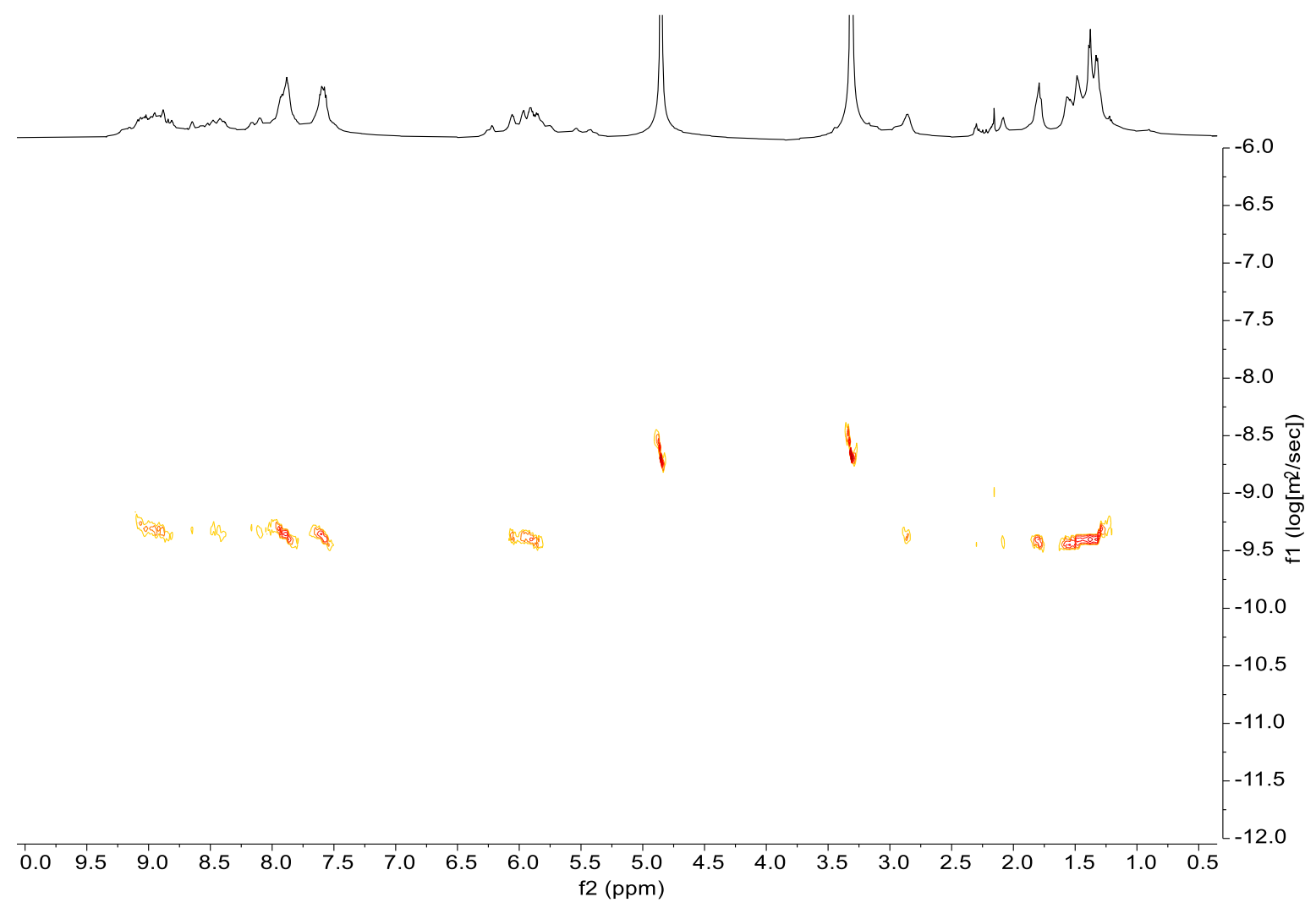

Figure S14. DOSY NMR spectrum of Zn-Phenyl Prism $\left(\mathrm{CD}_{3} \mathrm{OD}, 500 \mathrm{MHz}\right)$. 


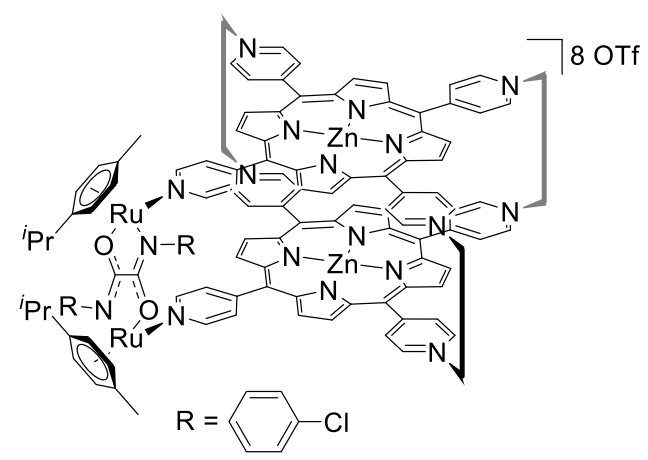

Aromatic

pyridyl, pyrrolic, and phenylene

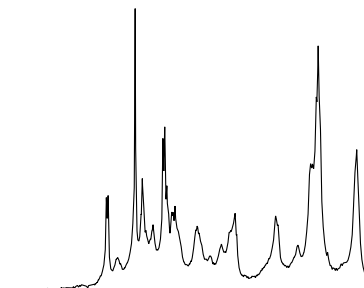

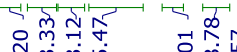

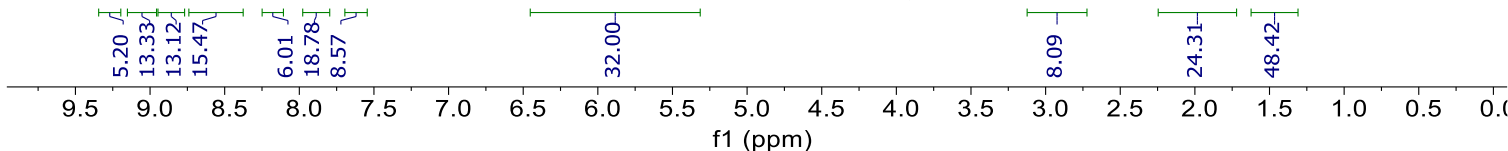

Aromatic p-cymene

Toure S15. ${ }^{1} \mathrm{H}$ NMR of $\mathbf{Z n}$-Chloro Prism $\left(\mathrm{CD}_{3} \mathrm{OD}, 500 \mathrm{MHz}\right)$. Resonances at 4.85 and $3.31 \mathrm{ppm}$ correspond to $\mathrm{H}_{2} \mathrm{O}$ and $\mathrm{CH}_{3} \mathrm{OH}$. Quartet at 3.49 and triplet at $1.18 \mathrm{ppm}$ correspond to diethyl ether used to precipitate. All other resonances can be attributed to the intact prism.

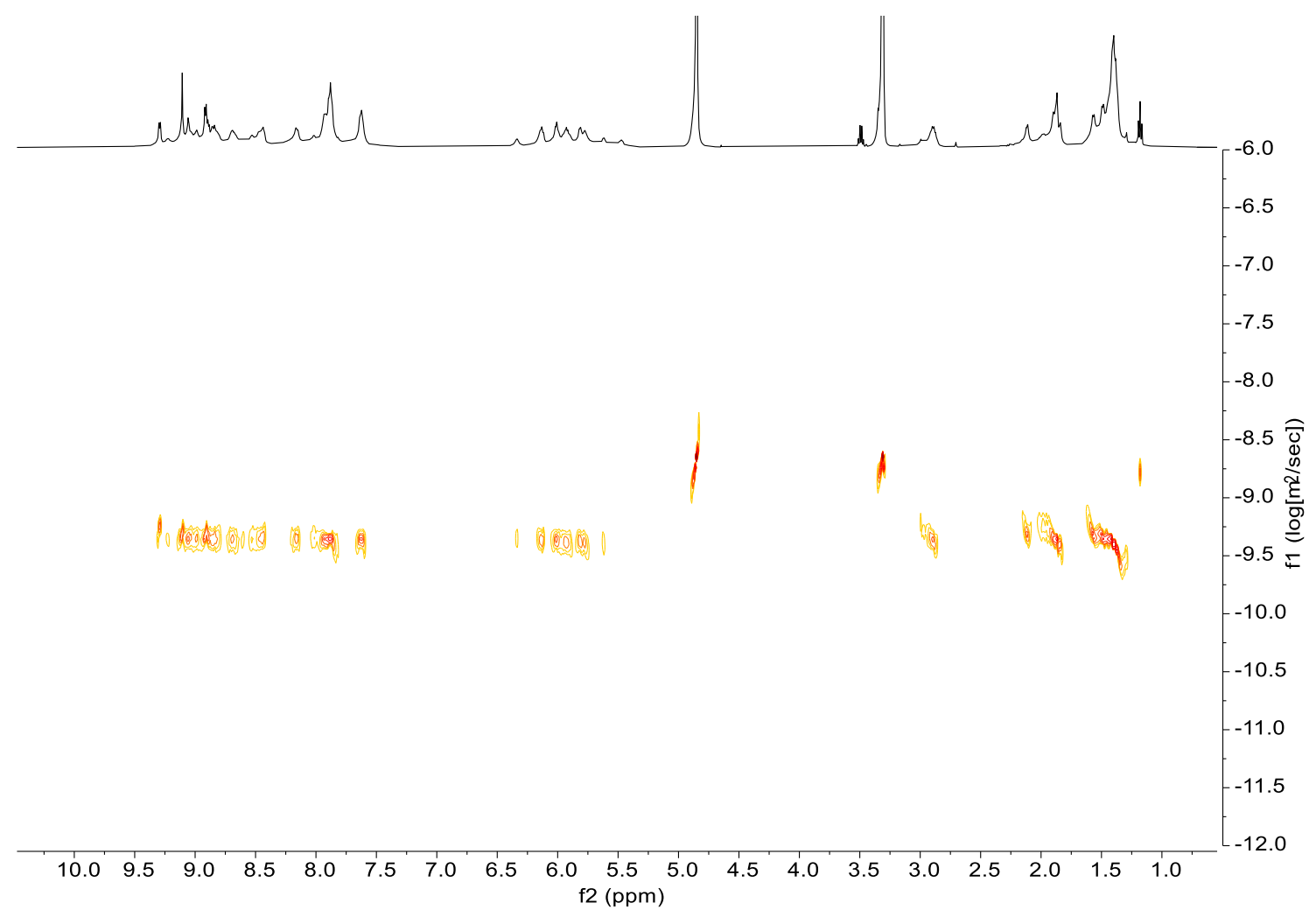

Figure S16. DOSY NMR spectrum of Zn-Chloro Prism $\left(\mathrm{CD}_{3} \mathrm{OD}, 500 \mathrm{MHz}\right)$. 


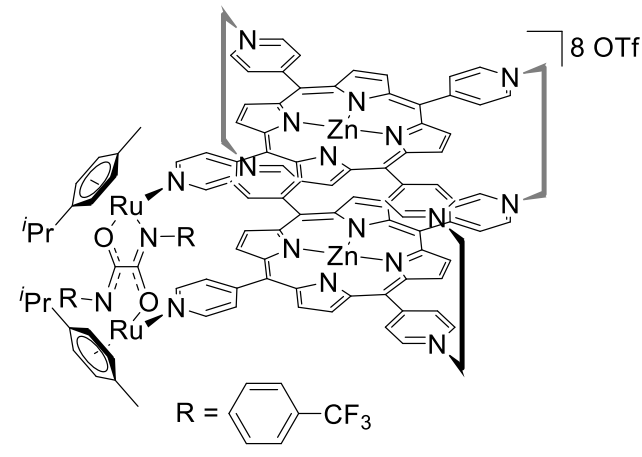

Aromatic

pyridyl, pyrrolic, and phenylene

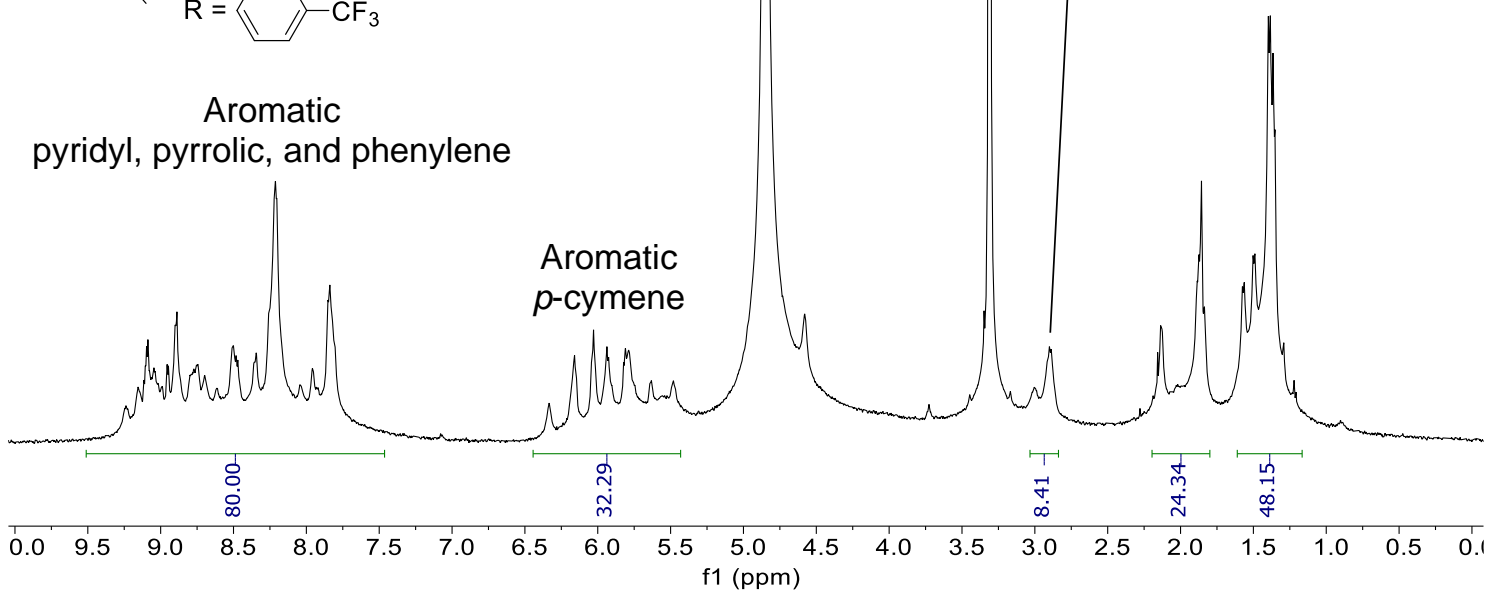

p-cymene iso-propyl

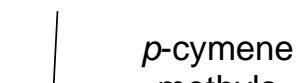
methyls

Figure S17. ${ }^{1} \mathrm{H}$ NMR of $\mathbf{Z n - C F} 3$ Prism $\left(\mathrm{CD}_{3} \mathrm{OD}, 500 \mathrm{MHz}\right)$. Resonances at 4.85 and 3.31 ppm correspond to $\mathrm{H}_{2} \mathrm{O}$ and $\mathrm{CH}_{3} \mathrm{OH}$. All other resonances can be attributed to the intact prism.

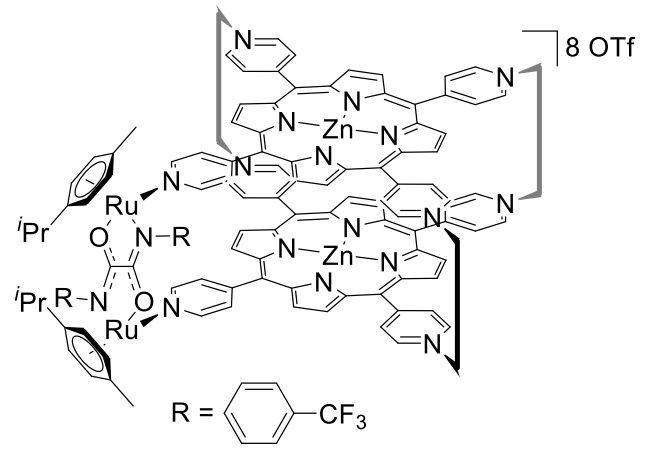

$\mathrm{OTf}^{-}$

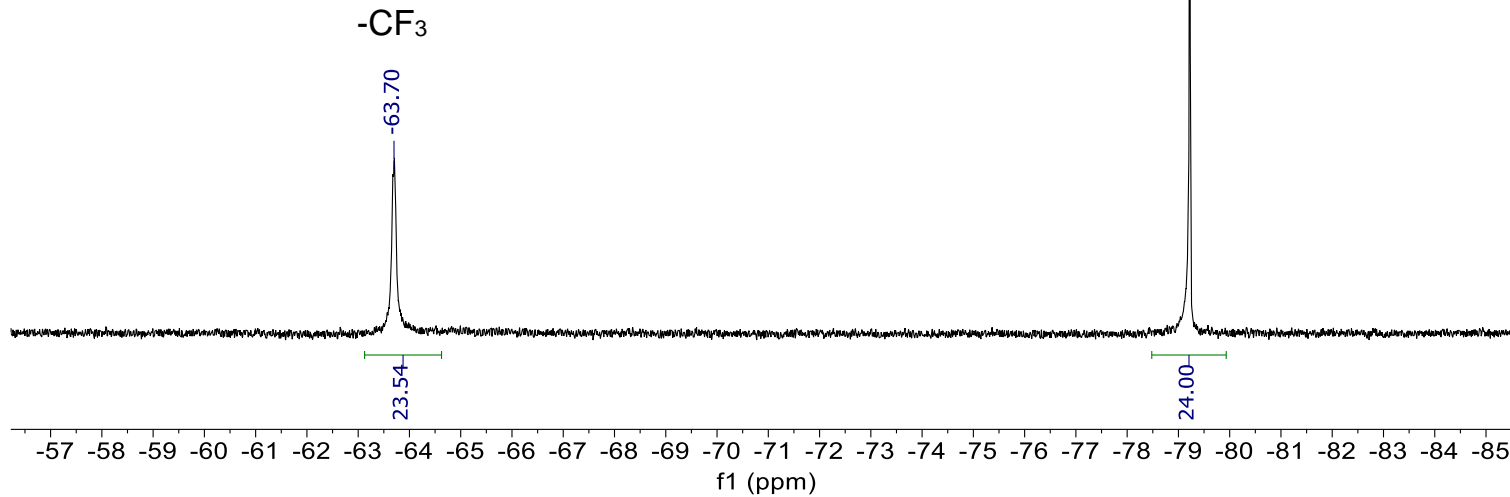

Figure S18. ${ }^{19} \mathrm{~F}\left\{{ }^{1} \mathrm{H}\right\}$ NMR of $\mathbf{Z n}-\mathbf{C F}_{3}$ Prism $\left(\mathrm{CD}_{3} \mathrm{OD}, 282 \mathrm{MHz}\right)$. 


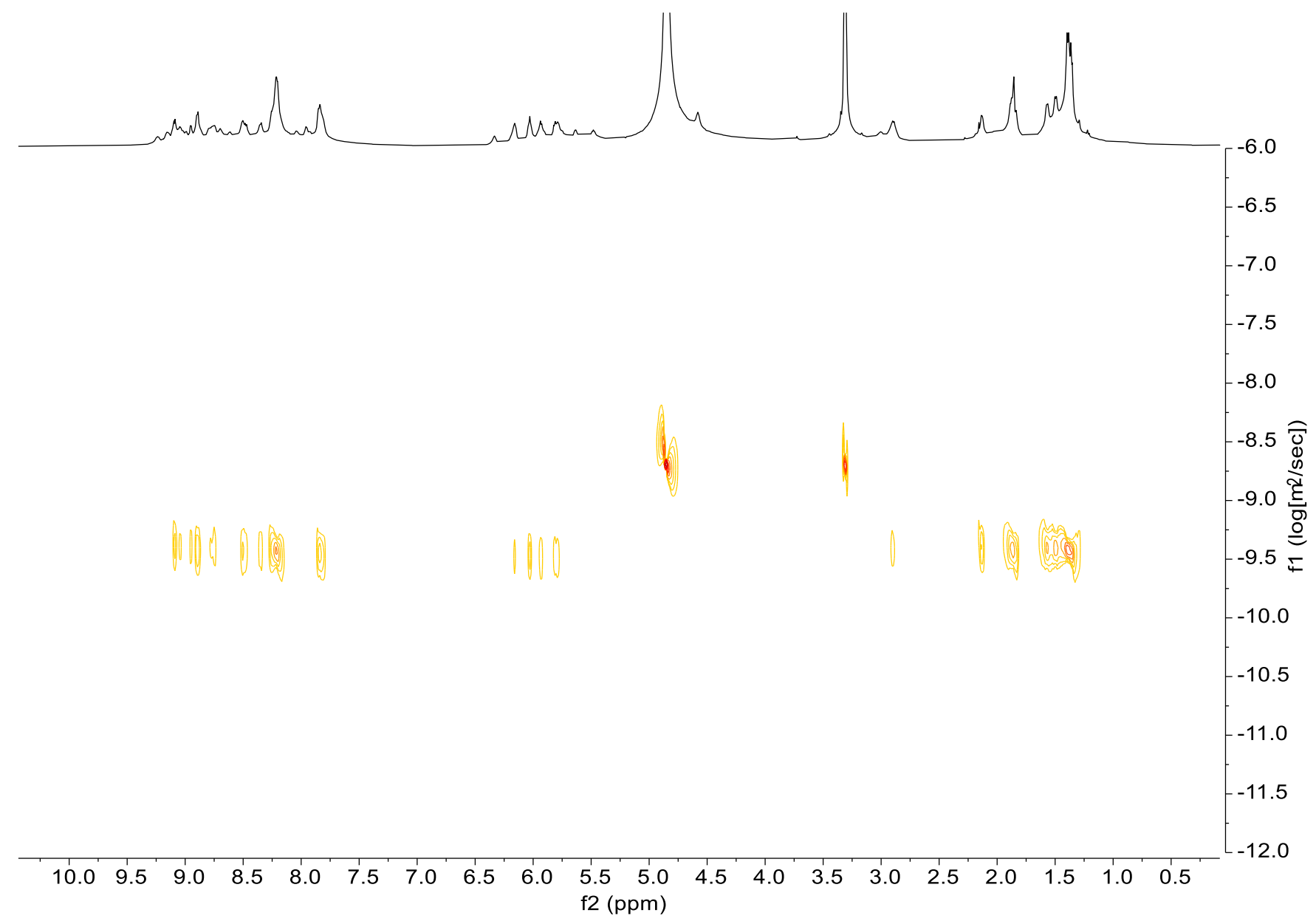

Figure S19. DOSY NMR spectrum of $\mathbf{Z n - C F} 3$ Prism $\left(\mathrm{CD}_{3} \mathrm{OD}, 500 \mathrm{MHz}\right)$.

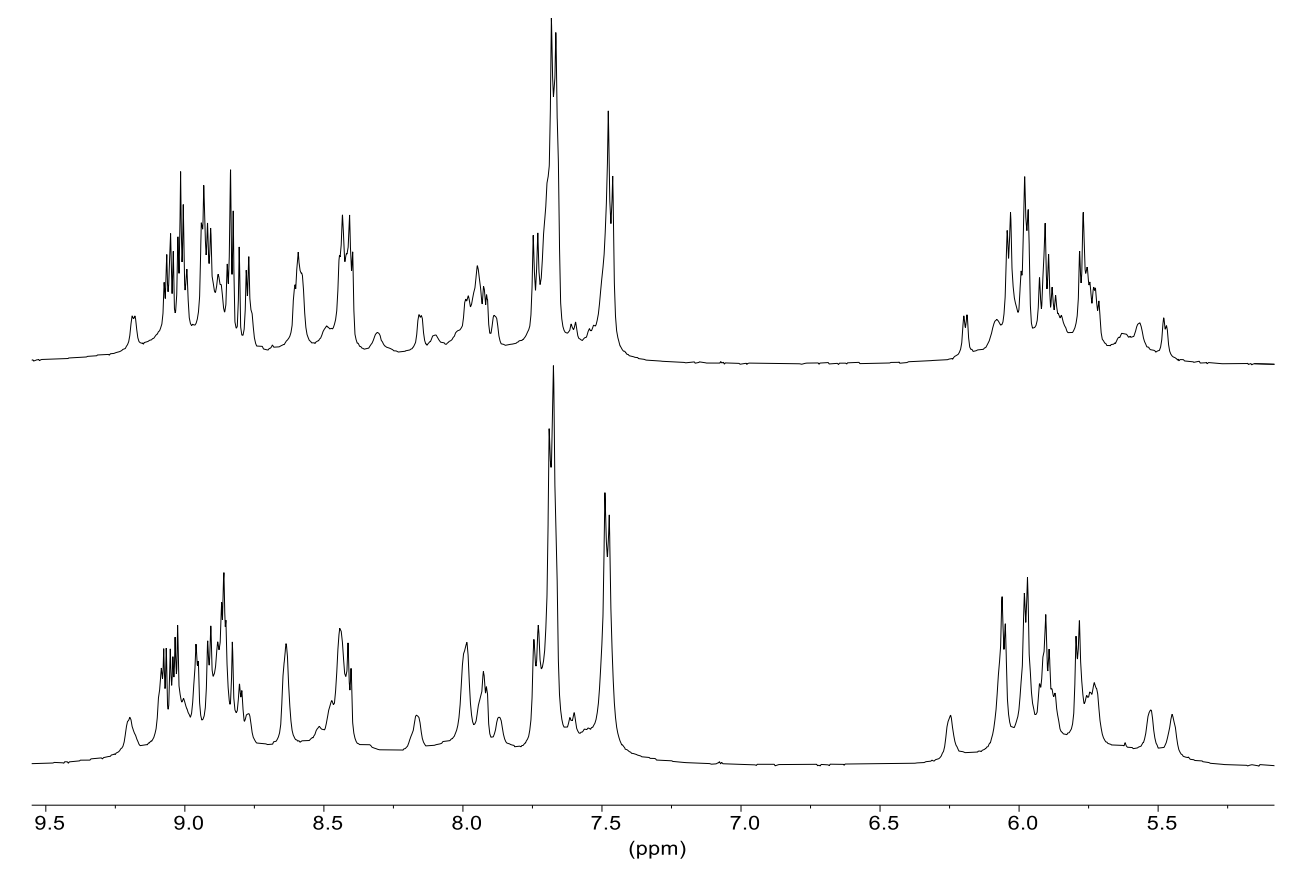

Figure S20. VT-NMR of Zn-Tolyl aromatic region at $25^{\circ} \mathrm{C}$ (bottom) and $50^{\circ} \mathrm{C}$ (top) (CD3OD, $500 \mathrm{MHz}$ ). 

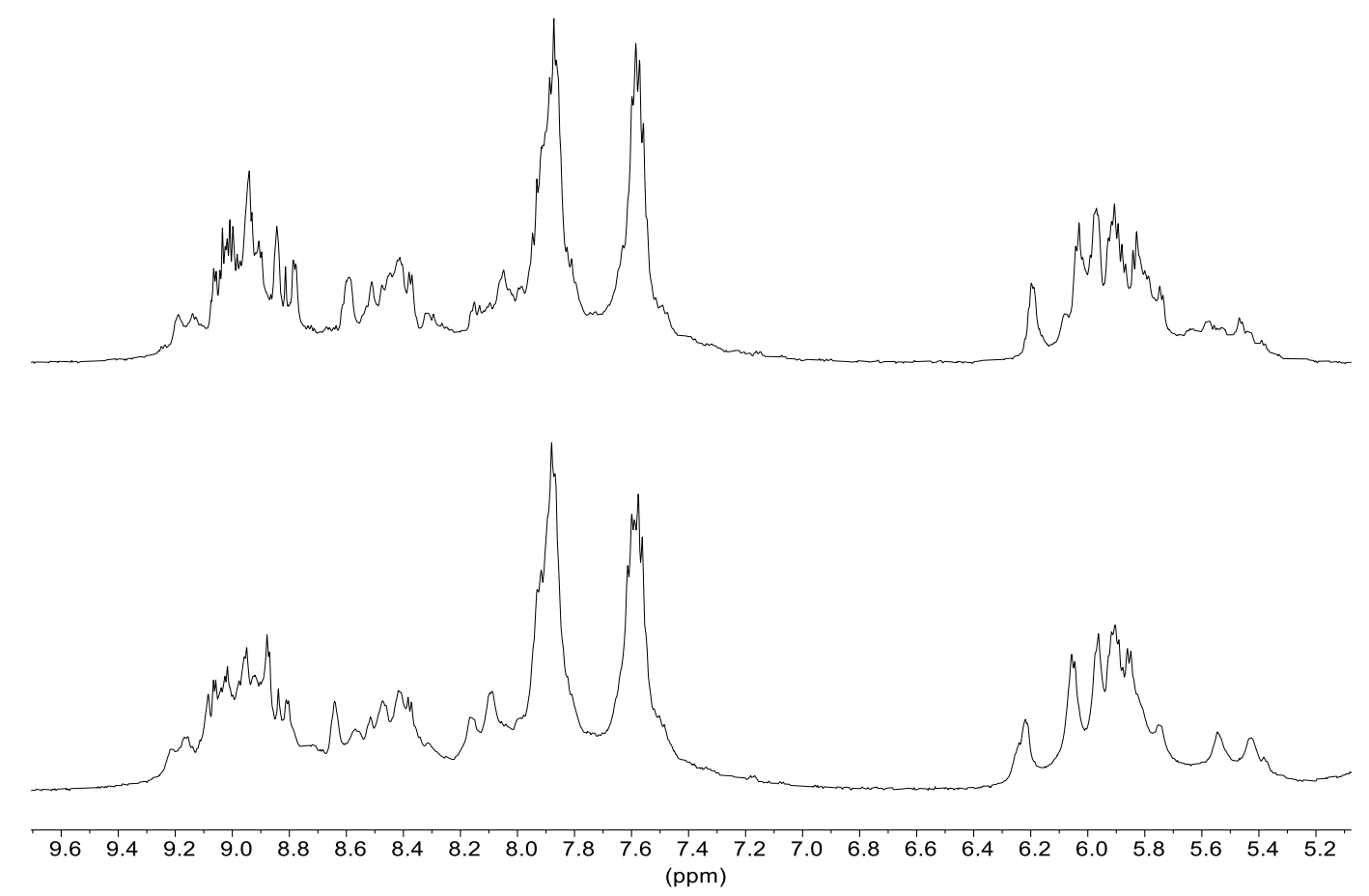

Figure S21. VT-NMR of Zn-Phenyl aromatic region at $25^{\circ} \mathrm{C}$ (bottom) and $50^{\circ} \mathrm{C}$ (top) $\left(\mathrm{CD}_{3} \mathrm{OD}, 500 \mathrm{MHz}\right.$ ).
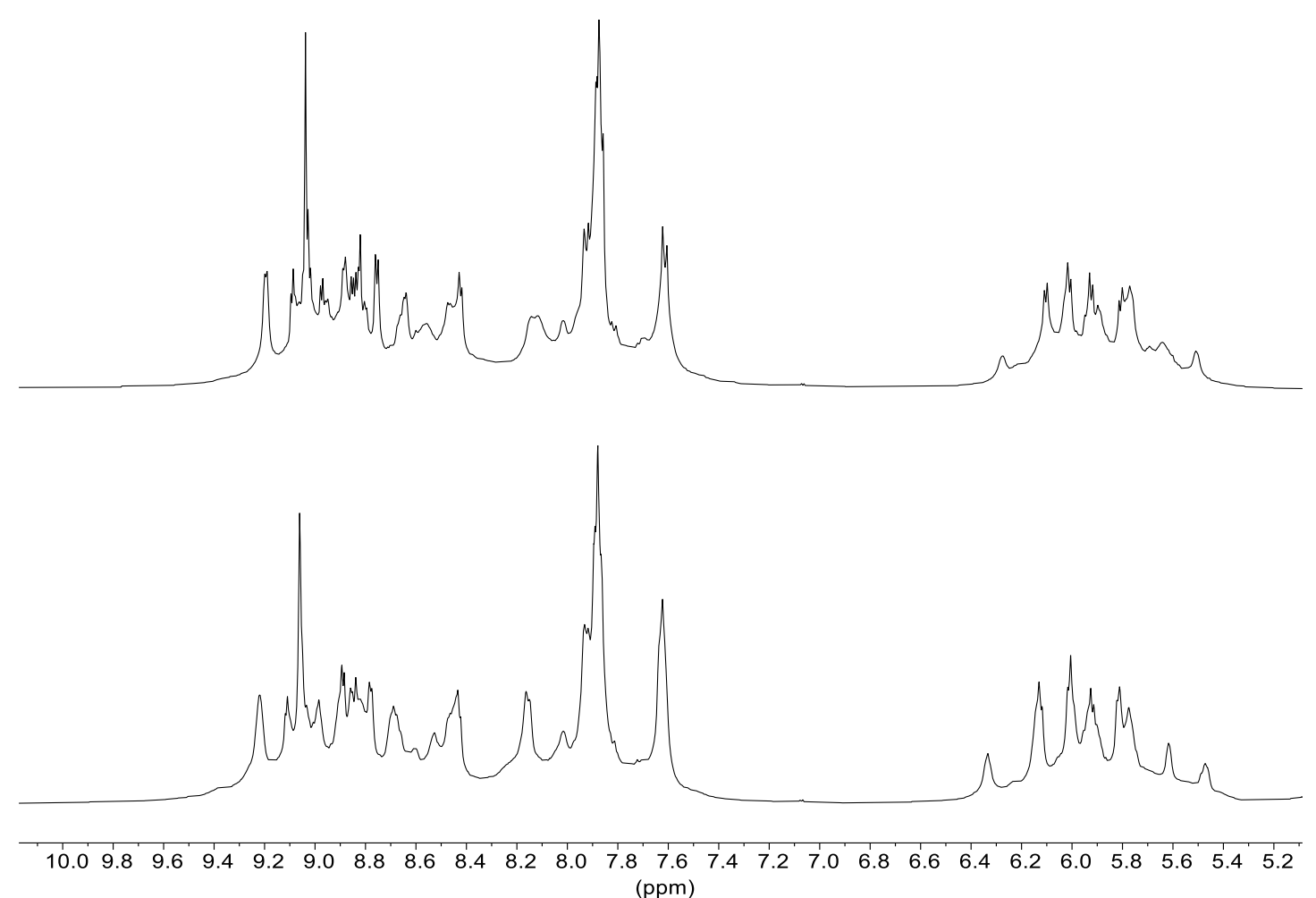

Figure S22. VT-NMR of Zn-Chloro aromatic region at $25^{\circ} \mathrm{C}$ (bottom) and $50^{\circ} \mathrm{C}$ (top) $\left(\mathrm{CD}_{3} \mathrm{OD}, 500 \mathrm{MHz}\right.$ ). 


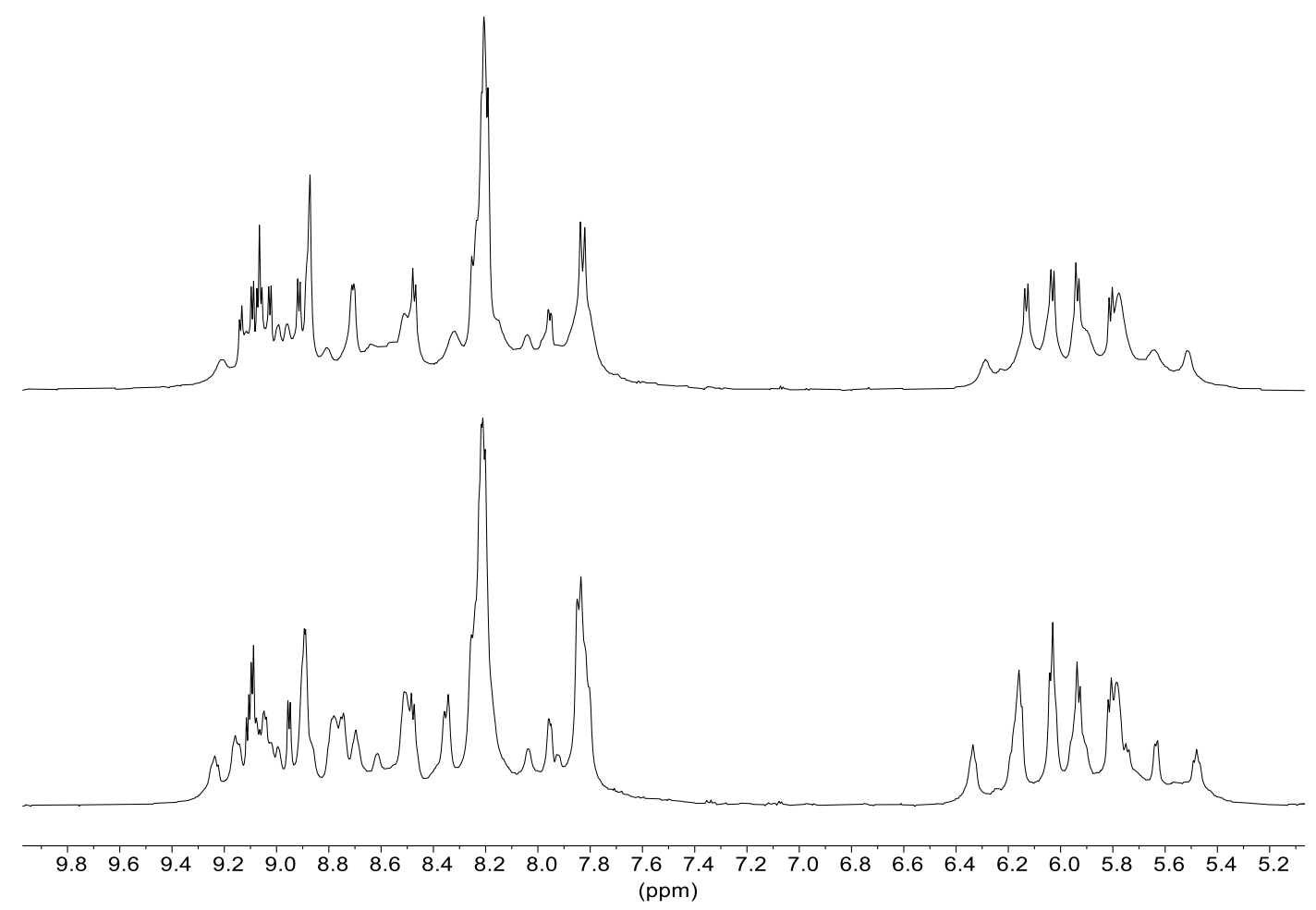

Figure S23. VT-NMR of $\mathbf{Z n}-\mathbf{C F}_{3}$ aromatic region at $25^{\circ} \mathrm{C}$ (bottom) and $50^{\circ} \mathrm{C}$ (top) $\left(\mathrm{CD}_{3} \mathrm{OD}, 500 \mathrm{MHz}\right.$ ).

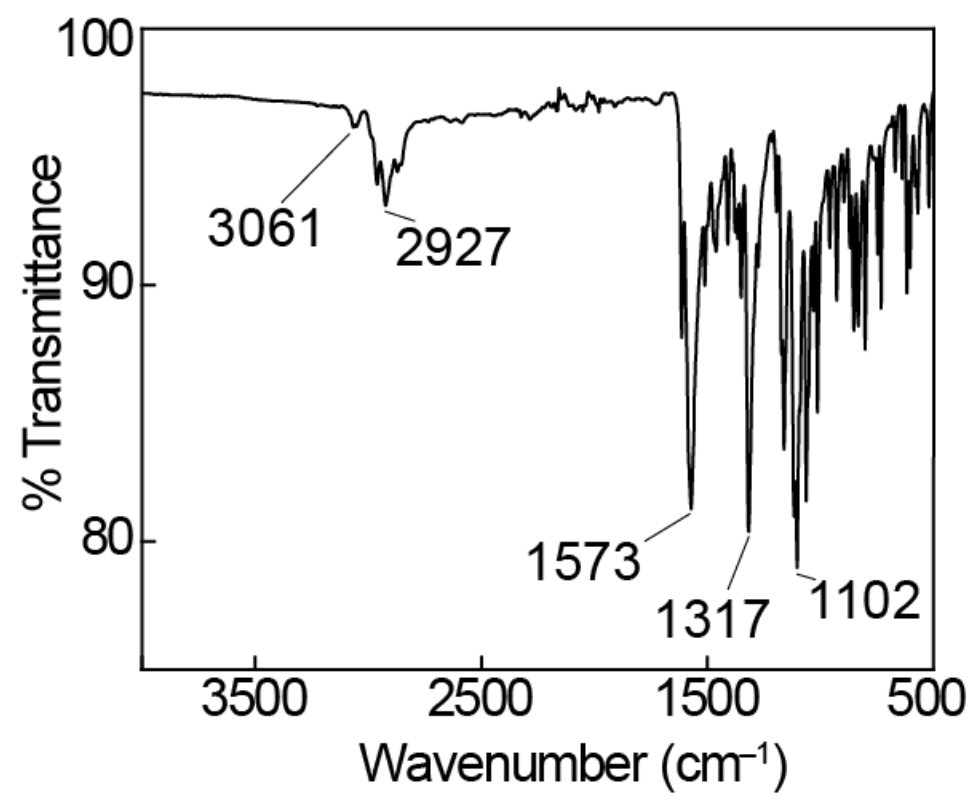

Figure S24. FT-IR spectrum of Ru-CF 3 clip. Spectrum was acquired on a neat powder averaging 16 scans. 


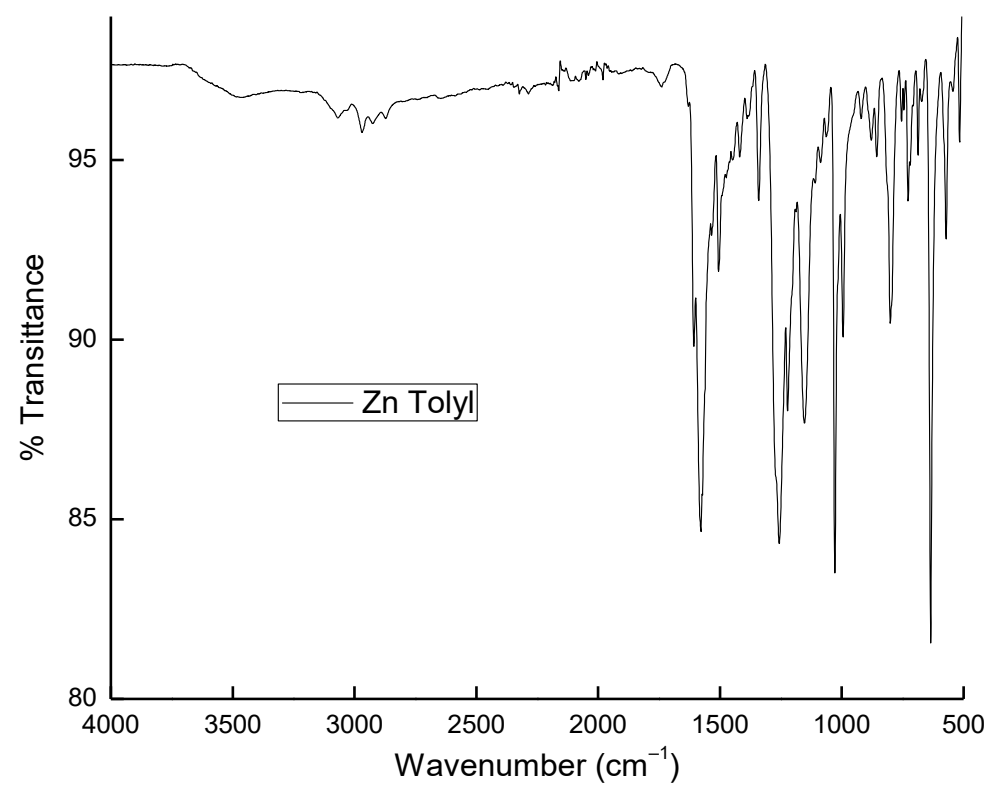

Figure S25. FT-IR spectrum of Zn-Tolyl prism. Spectrum was acquired on neat powder using an ATR accessory. 16 Scans averaged.

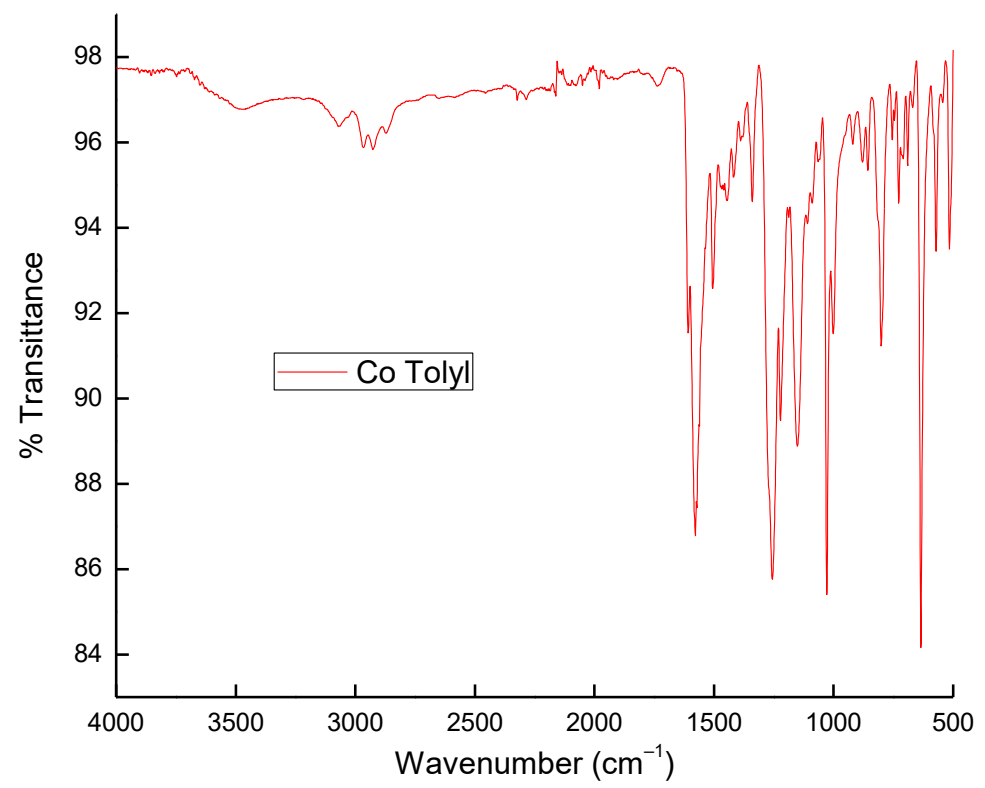

Figure S26. FT-IR spectrum of Co-Tolyl prism. Spectrum was acquired on neat powder using an ATR accessory. 16 Scans averaged. 


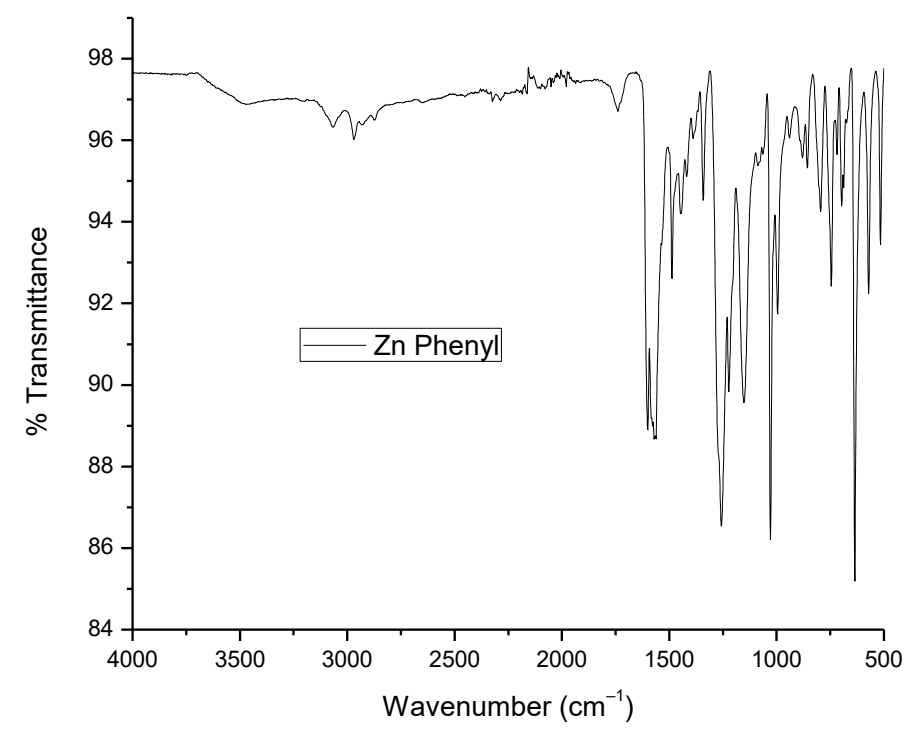

Figure S27. FT-IR spectrum of Zn-Phenyl prism. Spectrum was acquired on neat powder using an ATR accessory. 16 Scans averaged.

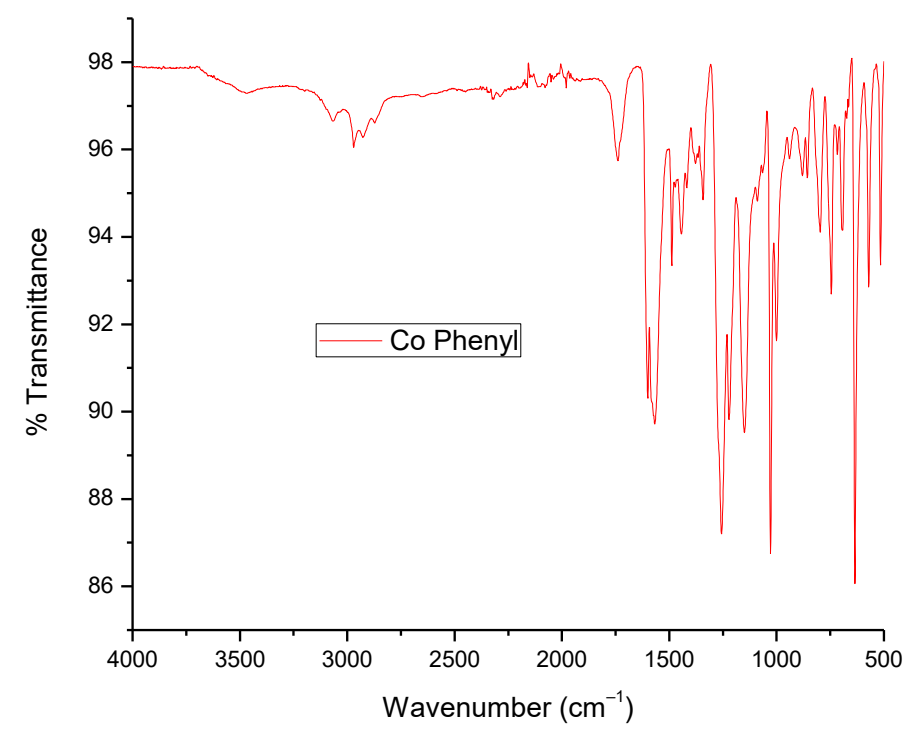

Figure S28. FT-IR spectrum of Co-Phenyl prism. Spectrum was acquired on neat powder using an ATR accessory. 16 Scans averaged. 


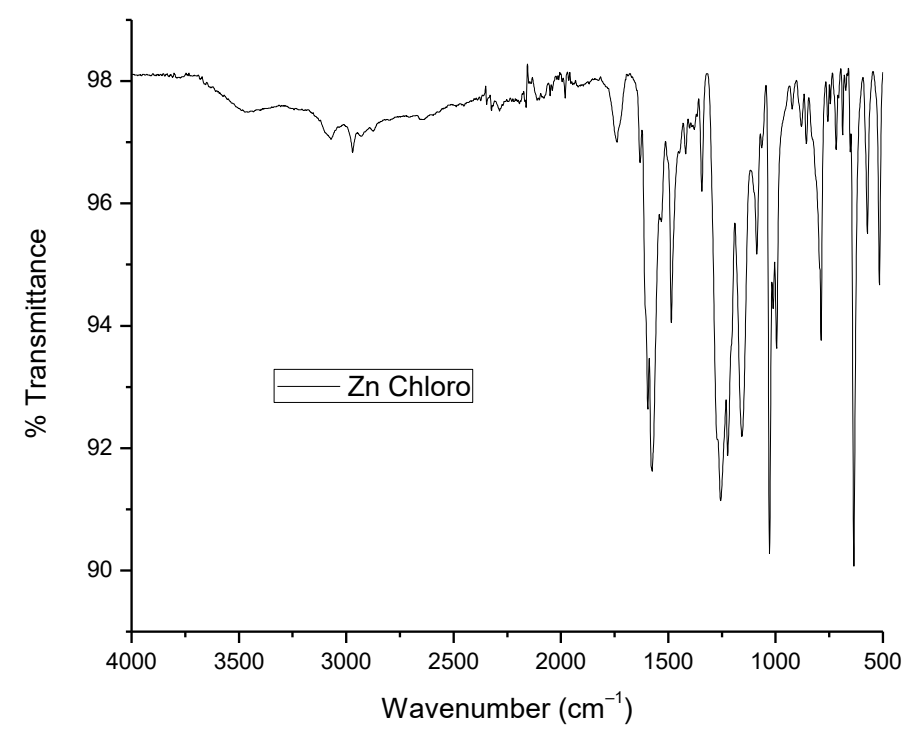

Figure S29. FT-IR spectrum of Zn-Chloro prism. Spectrum was acquired on neat powder using an ATR accessory. 16 Scans averaged.

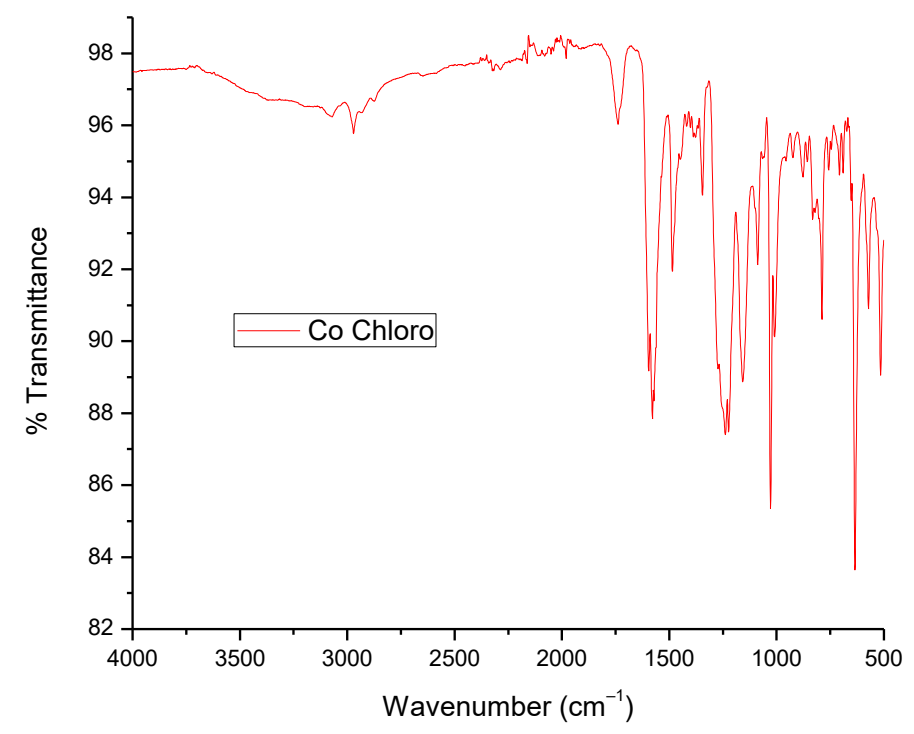

Figure S30. FT-IR spectrum of Co-Chloro prism. Spectrum was acquired on neat powder using an ATR accessory. 16 Scans averaged. 


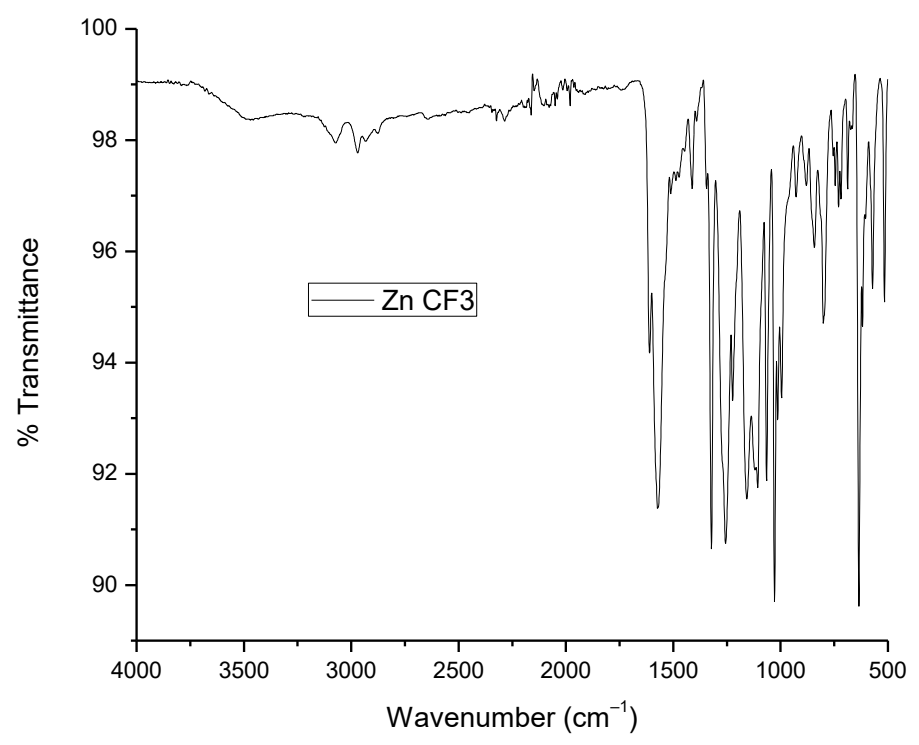

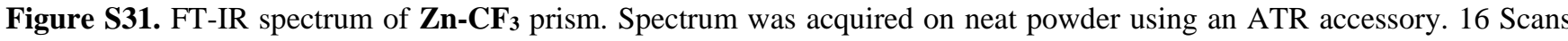
averaged.

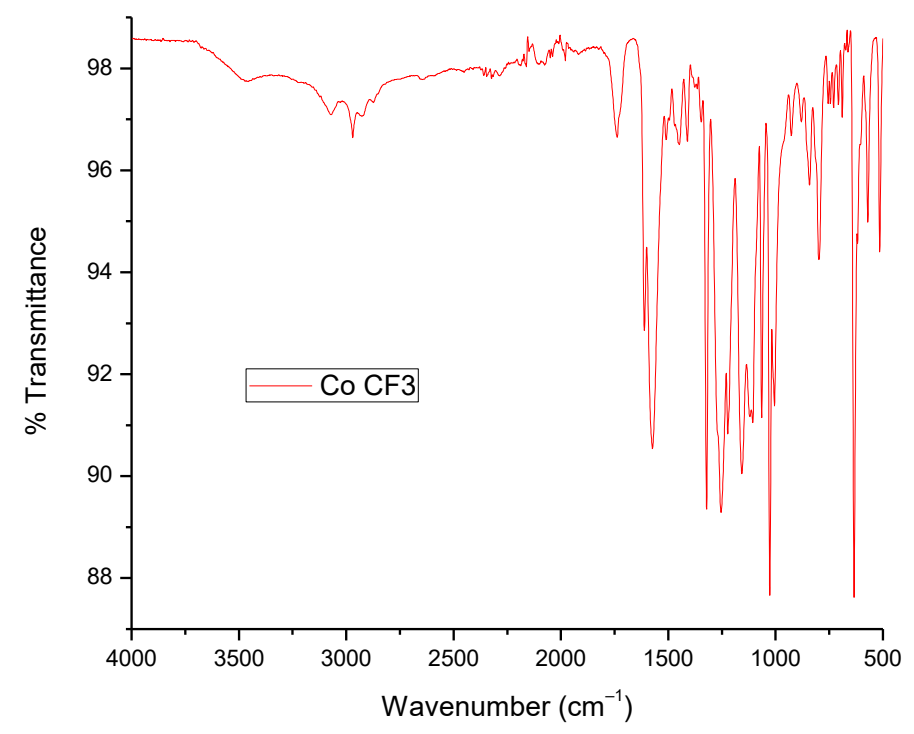

Figure S32. FT-IR spectrum of Co-CF $\mathbf{3}$ prism. Spectrum was acquired on neat powder using an ATR accessory. 16 Scans averaged. 

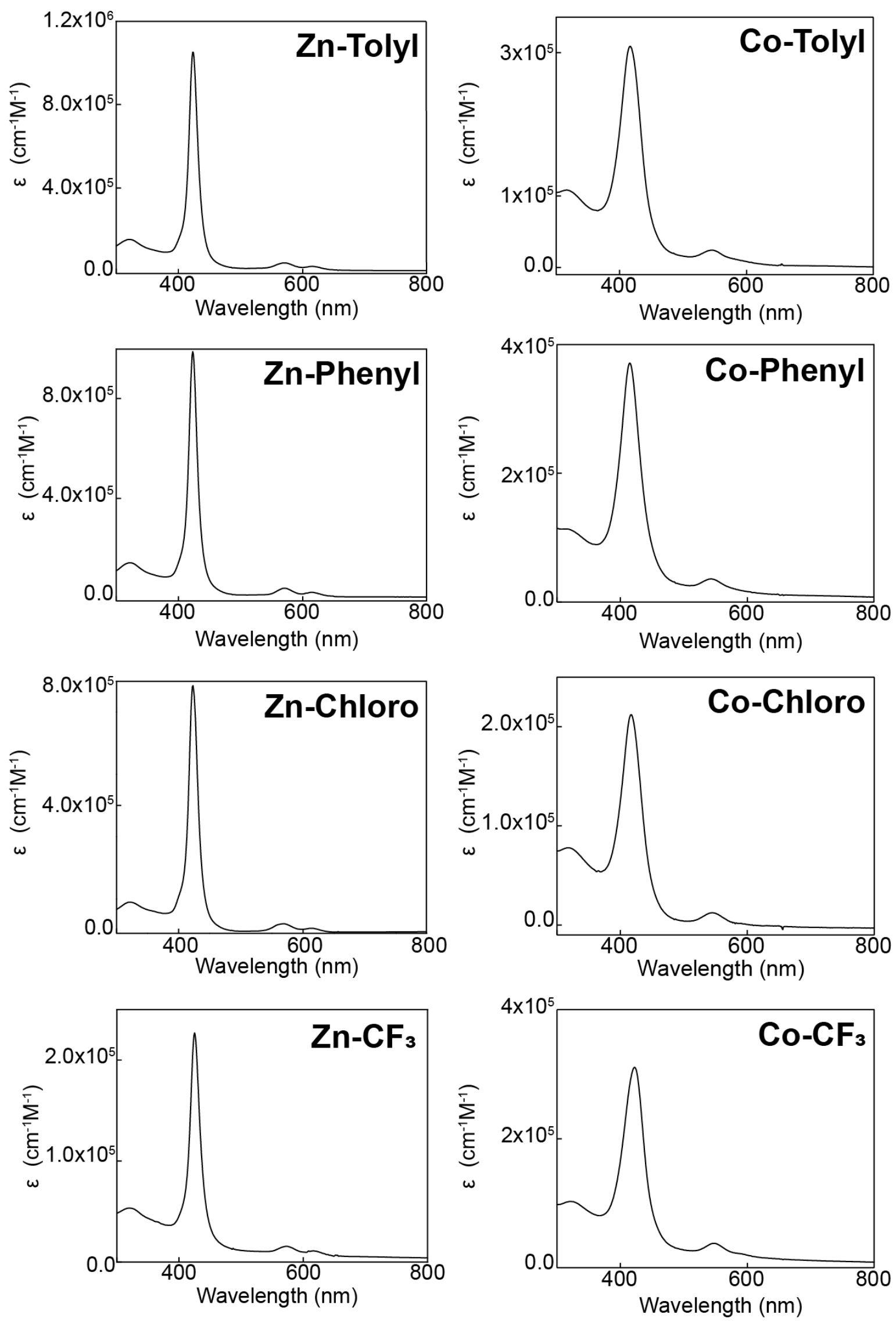

Figure S33. UV-Vis spectra of functionalized prisms. Data collected in methanol in a $1 \mathrm{~cm}$ path length quartz cuvette at room temperature. 


\section{Crystallographic Details}

Crystals of $\mathbf{R u}-\mathbf{C F}_{3}$ were grown by slow evaporation of a solution in $\mathrm{CDCl}_{3}$ in an $\mathrm{NMR}$ tube. The orange prisms de-solvated rapidly and required the use of a cooled microscope stage to allow sufficient time to select a suitable crystal. The datum crystal was mounted on a MiTeGen loop using N-Paratone oil. Data was collected on a Bruker D8 Venture diffractometer equipped with Photon-100 detector, and a sealed-tube Mo-target X-ray source. A series of $\Phi$ and $\omega$ scans were used to cover reciprocal space. Data refinement was routine. Integration and scaling of the data was performed in APEX3. ${ }^{3}$ Structure solution was obtained using SHELXS via the Patterson method, implemented through Olex2. ${ }^{4-5}$ Least-squares refinement was performed using SHELXL. ${ }^{6} \mathbf{R u}-\mathbf{C F}_{3}$ crystallized in the triclinic $P-1$ space group and resides on the inversion center special position, thus $\mathrm{Z}^{\prime}$ is 0.5 and there is one formula unit per unit cell $(\mathrm{Z}=1)$ (see Main Text for ellipsoid plot). Additionally, there were 4 cocrystallized $\mathrm{CHCl}_{3}$ molecules that form a pseudo-layered structure with alternating layers of $\mathrm{CHCl}_{3}$ and $\mathbf{R u}-\mathbf{C F}_{3}$, this helps to explain the rapid crystal degradation when removed from the mother liquor. There was minor disorder in the $-\mathrm{CF}_{3}$ groups; however, it was found that the refinement metrics were almost identical between the disorder modeled vs. unmodeled structures.

Crystals of $\mathbf{Z n}-\mathbf{C F}_{3}$ were grown by vapor diffusion of diethyl ether onto a solution of $\mathbf{Z n}-\mathbf{C F}_{3}$ in $1: 1 \mathrm{CH}_{2} \mathrm{Cl}_{2}$ to $\mathrm{MeOH}_{\text {. Crystals }}$ were small and poorly diffracting, in order to acquire the best possible data set synchrotron radiation was used. Data was collected at the Advanced Light Source housed at Lawrence Berkeley National Labs on beamline 12.2.1. Diffraction beyond $1.0 \AA$ was unachievable; however, this is more than sufficient for the purposes of atomic connectivity and approximate metalmetal separation. Radiation wavelength was $0.7288 \AA$. Data was collected using a Bruker D8 Venture diffractometer equipped with a Photon-II detector. Structure solution was obtained using SHELXT. ${ }^{7}$ Refinement was carried out as outlines above; however, several challenges arose during refinement. $\mathbf{Z n -} \mathbf{C F}_{3}$ crystallizes in the $C 2 / c$ space group. The porphyrin-clip scaffold could easily be found in the electron density map, the oxamide $N$-functional groups, $p$-cymene ligands, and the triflate counterions proved to be a greater challenge. To better model the molecular clips in a chemically reasonable way, the coordinates of the $p$-cymene ligand and 4-trifluoromethylphenyl fragment from the structure of $\mathbf{R u}-\mathbf{C F} \mathbf{F}_{\mathbf{3}}$ were optimized computationally using the Gaussian software package using DFT at the B3LYP level of theory with the 6-311G(d,p) basis set. These geometries have been submitted to, and are available free of charge from, Idealized Molecular Geometry Library. ${ }^{8}$ Using FRAG and FEND commands these idealized geometries were used to refine against the experimental data with good agreement. Of the eight outer-sphere triflate counterions, four were identifiable and modeled. The remaining four were found in a solvent accessible void space and highly disordered. After many attempts at modeling them, even with idealized geometries, a stable model could not be achieved. With this in mind we have elected to apply a solvent mask to the structure after modeling the entirety of the prism and as many triflates as possible. Using Olex2 to implement a solvent mask, two void spaces were found, the first contained $148 \mathrm{e}^{-}$this is almost exactly two triflate counterions (146 $\mathrm{e}^{-}$in two triflate groups). Since $\mathrm{Z}^{\prime}=0.5$ in this structure, this assignment accounts for all 4 of the "un-modelable" triflate counterions with almost no residual electron density after. The second void space located contained $\sim 12 \mathrm{e}^{-}$which is consistent with a partial occupancy $(\sim 70 \%)$, disordered methanol molecule. Ultimately, the acquired data confirms that the desired cofacial porphyrin prism, $\mathbf{Z n - C F}$, was formed with the expected molecular arrangement. 
Table S1. Crystallographic Details

\begin{tabular}{|c|c|c|}
\hline & $\mathbf{R u}-\mathrm{CF}_{3}$ & $\mathrm{Zn}-\mathrm{CF}_{\mathbf{3}}$ \\
\hline CCDC Number & 2023497 & 2041164 \\
\hline Empirical formula & $\mathrm{C}_{36} \mathrm{H}_{36} \mathrm{Cl}_{2} \mathrm{~F}_{6} \mathrm{~N}_{2} \mathrm{O}_{2} \mathrm{Ru}_{2} \bullet 4 \mathrm{CHCl}_{3}$ & $\mathrm{C}_{224} \mathrm{H}_{192} \mathrm{~F}_{24} \mathrm{~N}_{24} \mathrm{O}_{10} \mathrm{Ru}_{8} \mathrm{Zn}_{2} \cdot 8 \mathrm{CF}_{3} \mathrm{O}_{3} \mathrm{~S}$ \\
\hline Formula weight & 1393.18 & 5371.58 \\
\hline Temperature (K) & 120 & 100 \\
\hline Crystal system & triclinic & monoclinic \\
\hline Space group & $P-1$ & $C 2 / c$ \\
\hline$a(\AA)$ & $9.1940(4)$ & $41.929(2)$ \\
\hline$b(\AA)$ & $12.4090(5)$ & $28.768(1)$ \\
\hline$c(\AA)$ & $13.3632(6)$ & $25.701(1)$ \\
\hline$\alpha\left(^{\circ}\right)$ & $67.152(1)$ & 90 \\
\hline$\beta\left(^{\circ}\right)$ & $70.251(2)$ & $127.726(1)$ \\
\hline$\gamma\left({ }^{\circ}\right)$ & $78.909(2)$ & 90 \\
\hline Volume $\left(\AA^{3}\right)$ & $1319.0(1)$ & $24520(2)$ \\
\hline $\mathrm{Z}$ & 1 & 4 \\
\hline$\rho_{\text {calc }}\left(\mathrm{g} \mathrm{cm}^{-3}\right)$ & 1.754 & 1.455 \\
\hline$\mu\left(\mathrm{mm}^{-1)}\right.$ & 1.340 & 0.795 \\
\hline $\mathrm{F}(000)$ & 690.0 & 10816.0 \\
\hline Crystal size $\left(\mathrm{mm}^{3}\right)$ & $0.23 \times 0.21 \times 0.13$ & $0.1 \times 0.1 \times 0.1$ \\
\hline Radiation & $\operatorname{MoK} \alpha(\lambda=0.71073)$ & $\operatorname{MoK} \alpha(\lambda=0.71073)$ \\
\hline $2 \Theta$ range for data collection $\left(^{\circ}\right)$ & 5.714 to 56.706 & 2.456 to 41.832 \\
\hline Index ranges & $-12 \leq \mathrm{h} \leq 11,-16 \leq \mathrm{k} \leq 16,-17 \leq 1 \leq 17$ & $-42 \leq \mathrm{h} \leq 41,-28 \leq \mathrm{k} \leq 28,-25 \leq 1 \leq 25$ \\
\hline Reflections collected & $2 \overline{6} 32 \overline{7}$ & 147684 \\
\hline Independent reflections & $6559\left[\mathrm{R}_{\mathrm{int}}=0.0344, \mathrm{R}_{\text {sigma }}=0.0338\right]$ & $12917[$ Rint $=0.1453$, Rsigma $=0.0478]$ \\
\hline Data/restraints/parameters & $6559 / 36 / 407$ & $12917 / 1738 / 1312$ \\
\hline Goodness-of-fit on $F^{2}$ & 1.066 & 1.555 \\
\hline Final $R$ indexes $[I>=2 \sigma(I)]$ & $\mathrm{R}_{1}=0.0368, \mathrm{wR}_{2}=0.0774$ & $\mathrm{R} 1=0.1500, \mathrm{wR} 2=0.4041$ \\
\hline Final $\mathrm{R}$ indexes [all data] & $\mathrm{R}_{1}=0.0483, \mathrm{wR}_{2}=0.0826$ & $\mathrm{R} 1=0.2043, \mathrm{wR} 2=0.4517$ \\
\hline Largest diff. peak/hole $\left(\mathrm{e}^{-} \AA^{-3}\right)$ & $1.40 /-1.50$ & $1.57 /-0.70$ \\
\hline
\end{tabular}

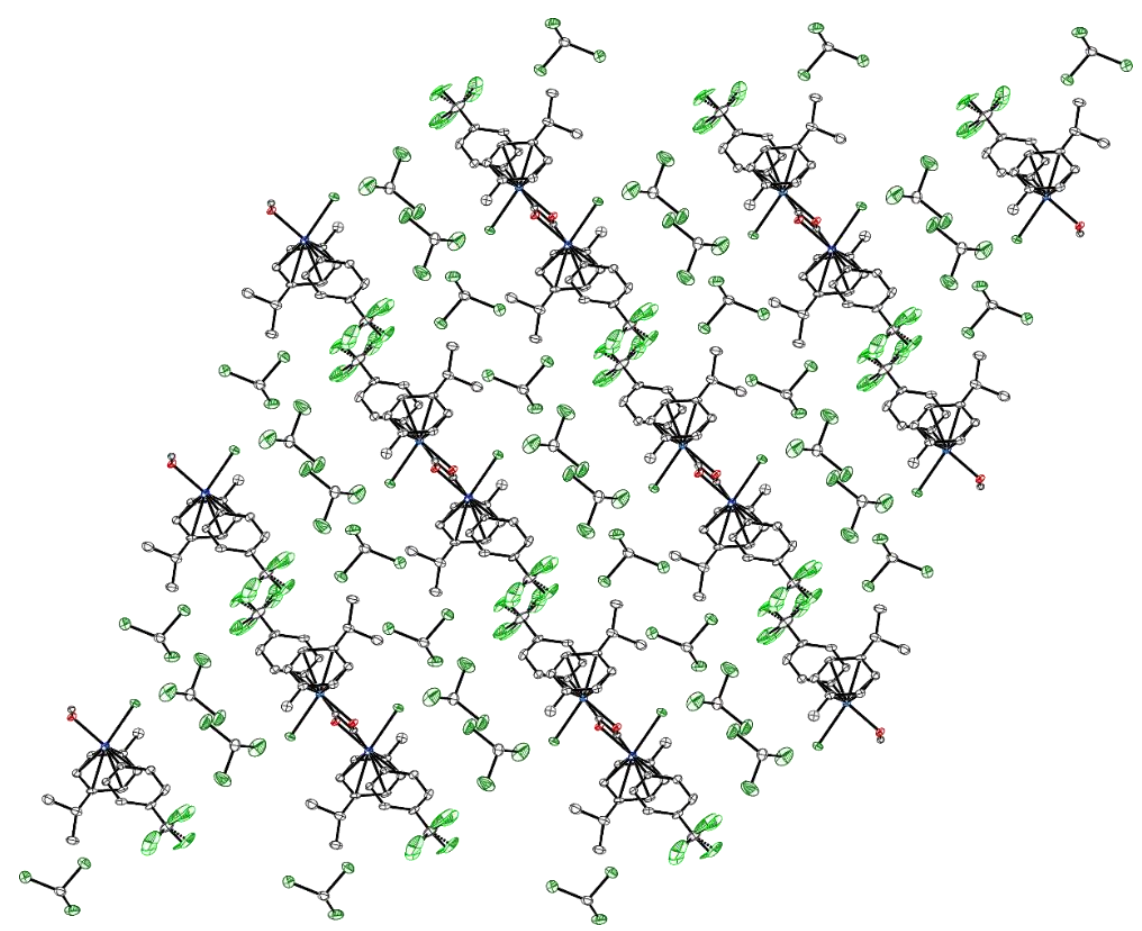

Figure S34. Illustration of packing in the crystal structure of Ru-CF3. The distinct layers of $\mathrm{CHCl}_{3}$ and $\mathbf{R u}-\mathbf{C F} 3 \mathbf{c a n}$ be seen running diagonally. 


\section{Electrochemical Experiments}

\section{General Procedures}

All electrochemical experiments were performed using BioLogic SP300 bipotentiostat. TBAPF 6 was recrystallized three times from ethanol and dried for at least 48 hours in vacuo before use in non-aqueous experiments. Acetonitrile was dried using a Pure Process Technology solvent system. For heterogeneous films in aqueous media nanopore water was used to prepare the $0.5 \mathrm{M} \mathrm{H}_{2} \mathrm{SO}_{4}$ solution which served as both the proton source as well as the electrolyte. A non-aqueous $\mathrm{Ag} / \mathrm{AgNO} \mathrm{H}_{3}$ reference electrode was used and a ferrocene standard was used to convert all non-aqueous potentials to $\mathrm{Fc}^{+/ 0}$ for homogeneous nonaqueous experiments. For aqueous experiments, $0.5 \mathrm{M} \mathrm{H}_{2} \mathrm{SO}_{4}$ in water was used as the solvent and supporting electrolyte. An aqueous $\mathrm{Ag} / \mathrm{AgCl}(3 \mathrm{M} \mathrm{KCl})$ reference electrode was used for heterogeneous film experiments in aqueous media. In all experiments a Pt wire counter electrode and glassy carbon working electrode were used. For hydrodynamic voltammetry, a Pine MSR rotator was used with a glass carbon disk, Pt ring, ring-disk electrode. Unless otherwise state, scan rates were 100 $\mathrm{mV} / \mathrm{sec}$ for $\mathrm{CVs}$ and $20 \mathrm{mV} / \mathrm{sec}$ for linear sweep voltammograms using in hydrodynamic voltammetry experiments.

\section{Homogeneous Electrochemical Experiments}

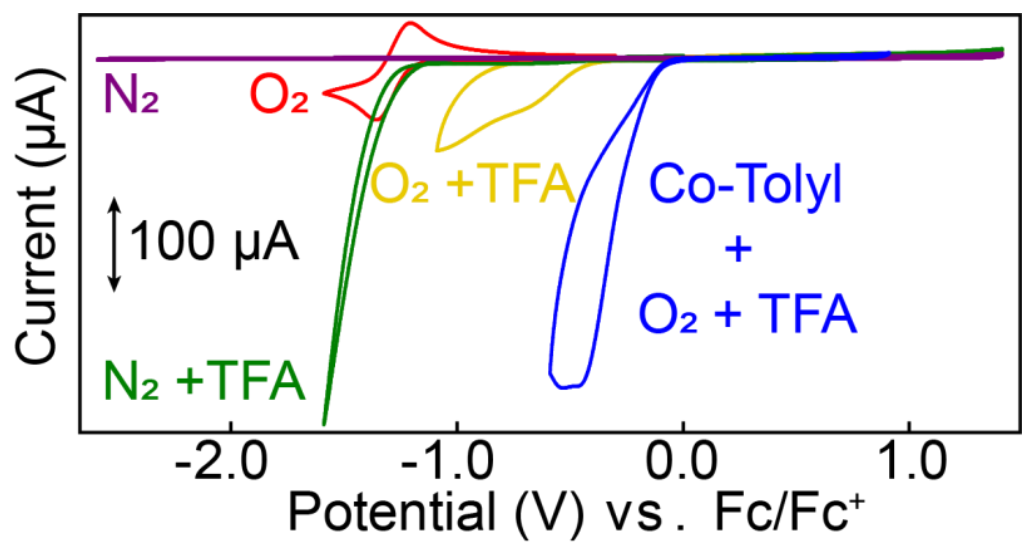

Figure S35. Electrochemical blanks under homogeneous conditions. Conditions: $100 \mathrm{mM}$ TBAPF6 in dry acetonitrile, glassy carbon working electrode, Pt-wire counter electrode, scan rate: $100 \mathrm{mV} / \mathrm{sec}$. (Purple) solution sparged with nitrogen, (Red) solution sparged with $\mathrm{O} 2$, the reversible feature is the formation of superoxide at the glassy carbon working electrode, (Green) solution sparged with $\mathrm{N} 2$ with $100 \mathrm{mM}$ trifluoroacetic acid added, the large feature is proton reduction, (Yellow) solution sparged with $\mathrm{O} 2$ with $100 \mathrm{mM}$ trifluoroacetic acid added, (Blue) Co-Tolyl under catalytic conditions is added for comparison. 


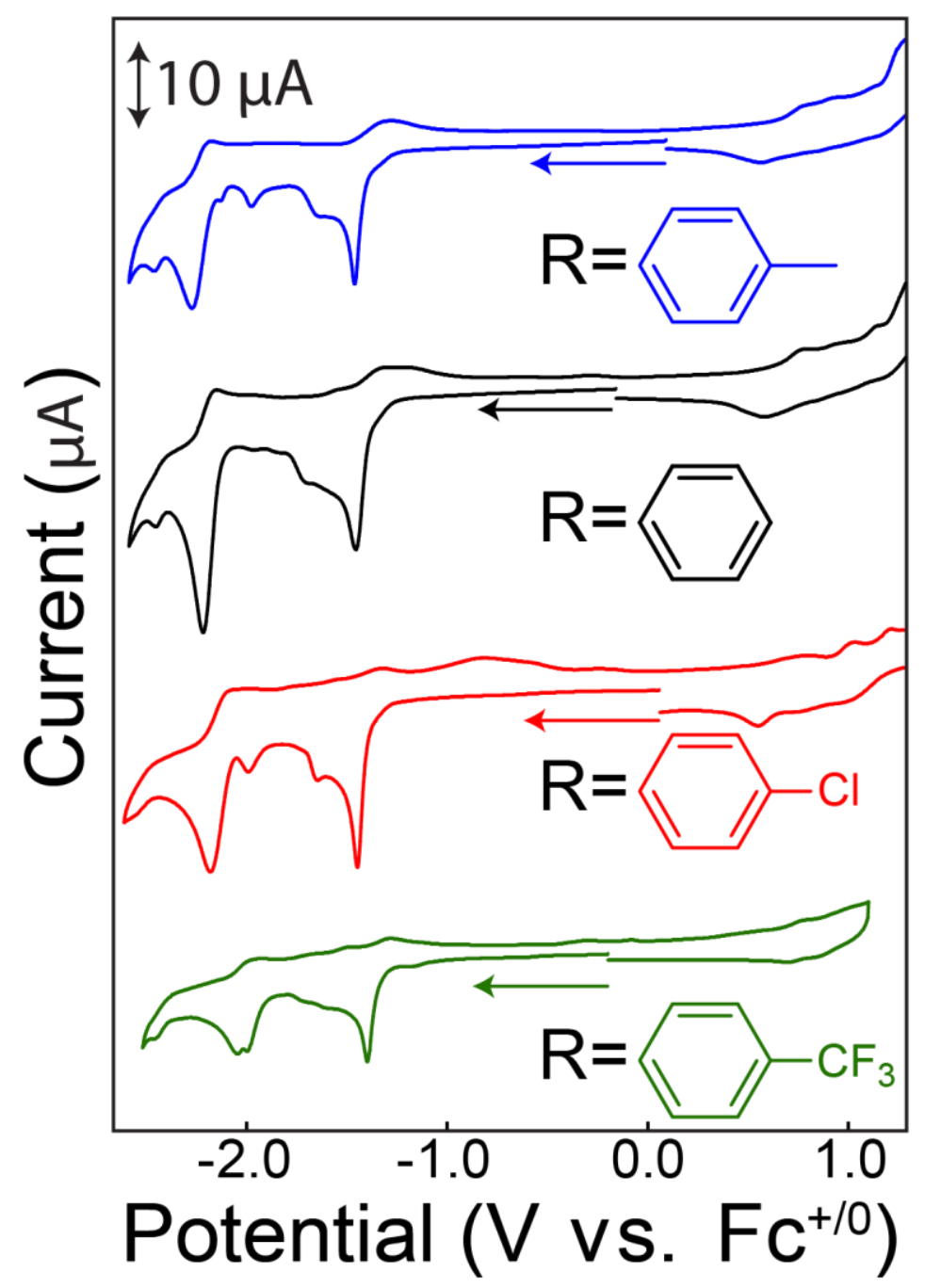

Figure S36. CV of Zn-prisms illustrating current responses. All CV were acquired at room temperature under N2 atmosphere with rigorously dry solvent. Prism concentration was $0.1 \mathrm{mM}$ with $100 \mathrm{mM}$ TBAPF $_{6}$ added as supporting electrolyte. Scan rate: $100 \mathrm{mV} / \mathrm{sec}$. 


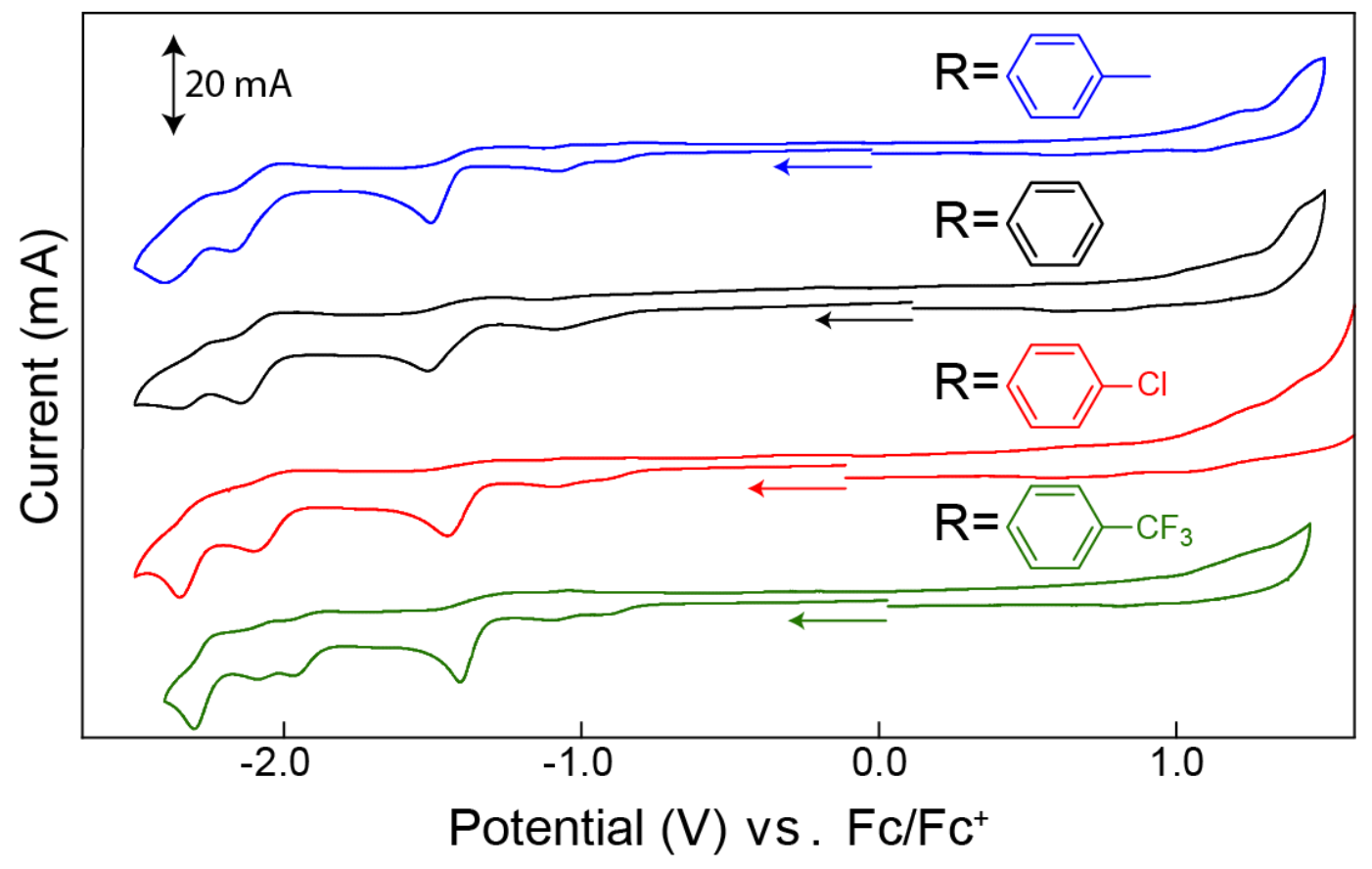

Figure S 37. CV of Co-prisms illustrating current responses. All CV were acquired at room temperature under N2 atmosphere with rigorously dry solvent. Prism concentration was $0.1 \mathrm{mM}$ with $100 \mathrm{mM}$ TBAPF6 added as supporting electrolyte. Scan rate: $100 \mathrm{mV} / \mathrm{sec}$. 


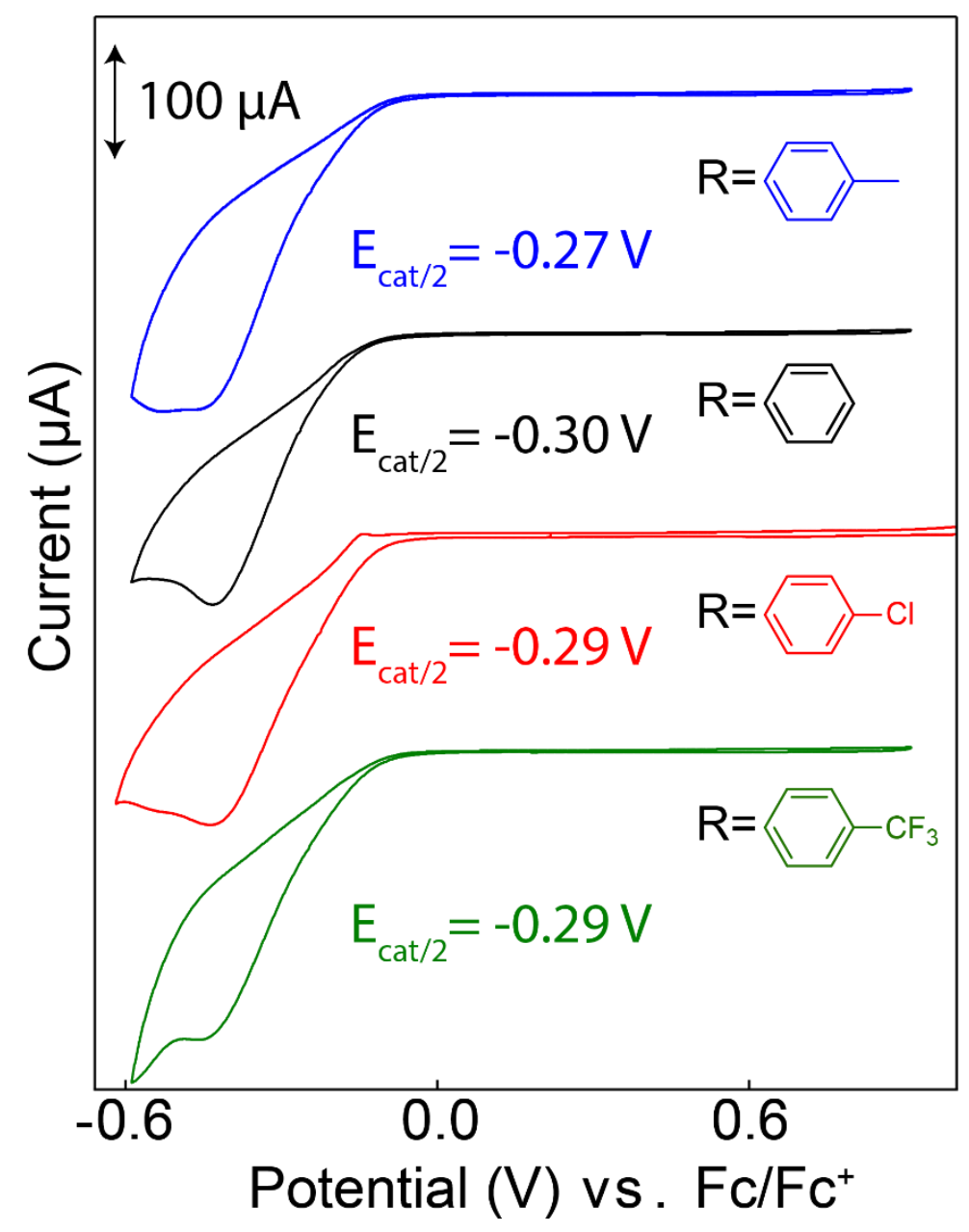

Figure S38. CV of Co-prisms $(0.1 \mathrm{mM})$ in acetonitrile with $100 \mathrm{mM} \mathrm{TBAPF}_{6}$ after sparging with $\mathrm{O}_{2}$, in the presences of 100 $\mathrm{mM}$ TFA. Scan rate: $100 \mathrm{mV} / \mathrm{sec}$. 

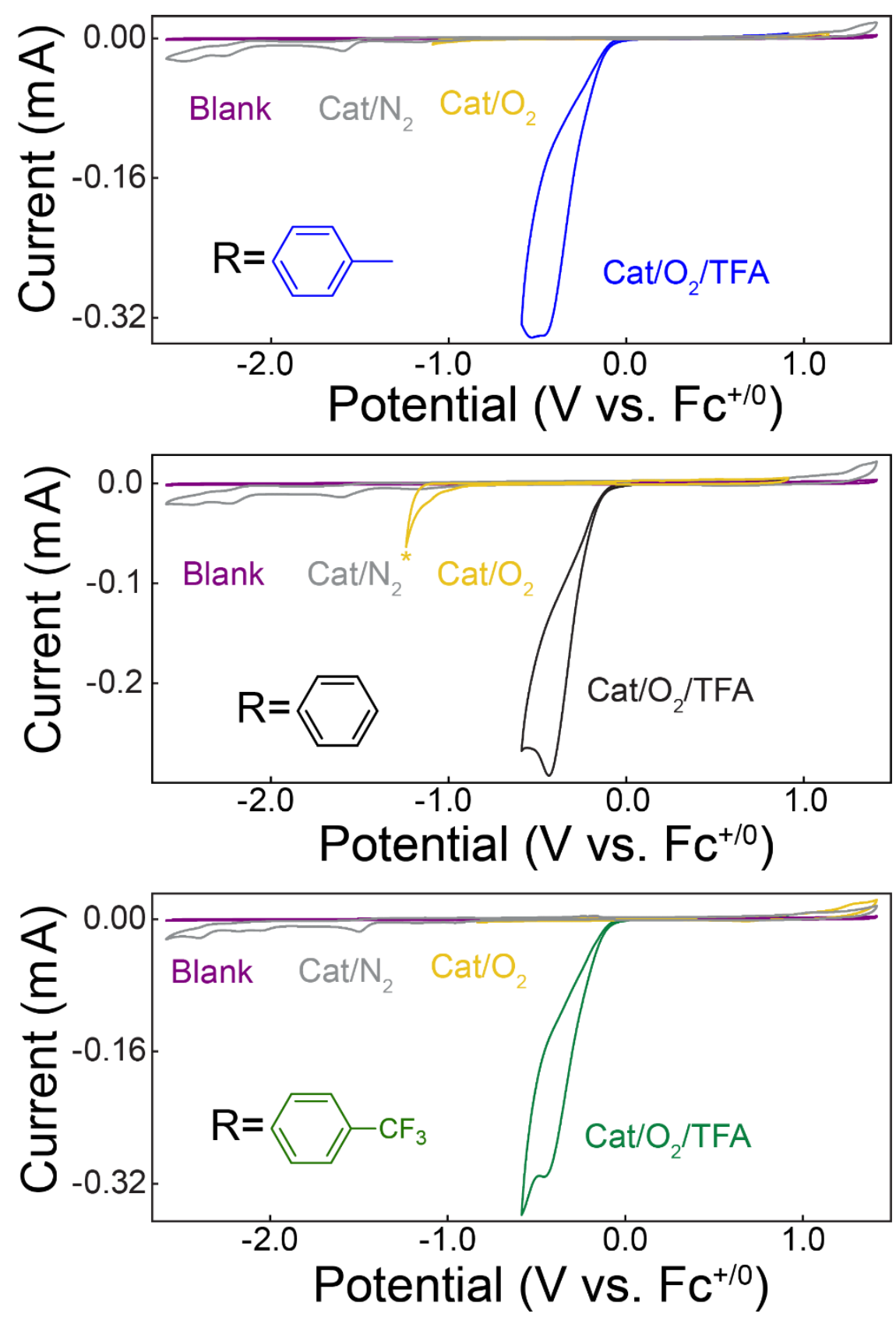

Figure S39. CV of Co-prisms under variable conditions. Blank (purple, $\mathrm{N}_{2}$ atmosphere), catalyst under inert conditions (gray, $0.1 \mathrm{mM}$ Co-prism, $\mathrm{N}_{2}$ atmosphere), catalyst with $\mathrm{O}_{2}$ (respective yellow, $0.1 \mathrm{mM}$ Co-prism, $\mathrm{O}_{2}$ atmosphere), and catalyst under catalytic conditions (respective color, $0.1 \mathrm{mM}$ Co-prism, $\mathrm{O}_{2}$ atmosphere, $100 \mathrm{mM}$ TFA). In all cases, dry acetonitrile with 100 $\mathrm{mM} \mathrm{TBAPF}{ }_{6}$ was used. * indicates the onset of superoxide formation at the glassy carbon working electrode. 

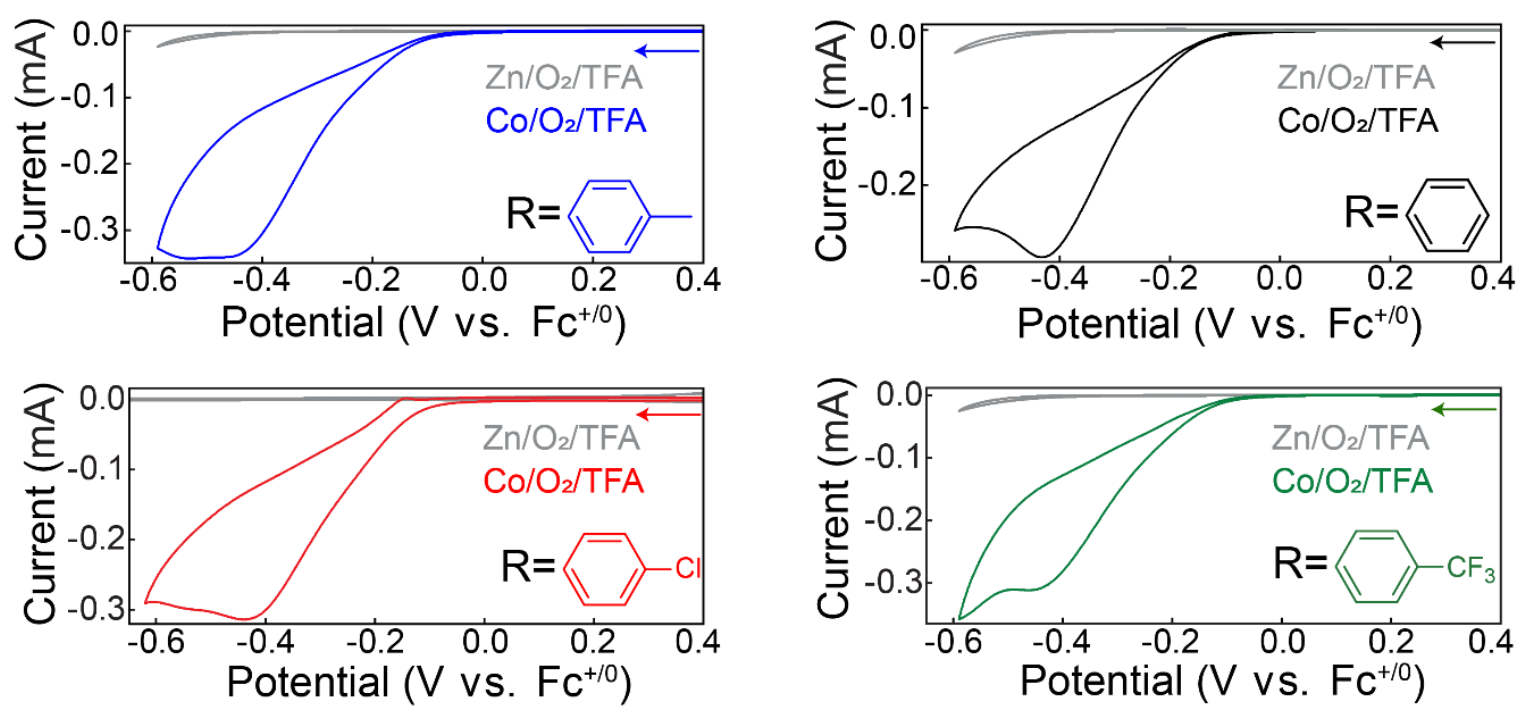

Figure S40. Comparison of Zn-Prisms and Co-Prisms $(0.1 \mathrm{mM})$ in acetonitrile $\left(100 \mathrm{mM} \mathrm{TBAPF}_{6}\right.$ supporting electrolyte) under catalytic conditions (100 mM TFA, sparged with $\mathrm{O}_{2}$ at $100 \mathrm{mV} / \mathrm{sec}$.

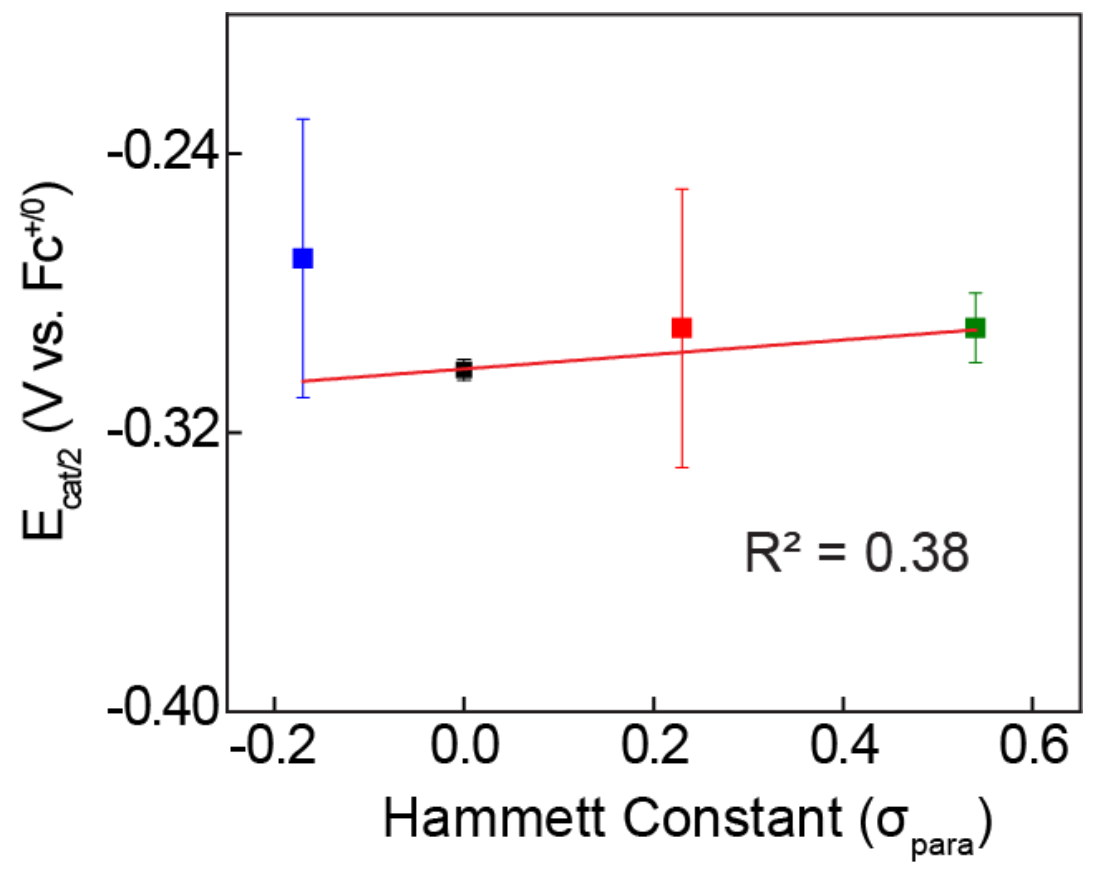

Figure S41. Homogeneous $E_{c a t / 2}$ vs Hammett constant for Functionalized prisms (Blue = Co-Tolyl, Black = Co-Phenyl, Red $=$ Co-Chloro, Green $\left.=\mathbf{C o}-\mathbf{C F}_{3}\right)$.

\section{Heterogeneous Film Electrochemical Experiments}

\section{Catalyst Ink Film Preparation}

In a 2-dram vial, $1 \mu \mathrm{mol}$ of catalyst (approximately $5.5 \mathrm{mg}$ ), $5.5 \mathrm{mg}$ of carbon black, $100 \mu \mathrm{L}$ of ethanol, and $500 \mu \mathrm{L}$ methanol were sonicated for 1 hour. The solvent was then evaporated in vacuo, followed by the addition of a $70 \mu \mathrm{L}$ Nafion solution $(5 \%$ $\mathrm{w} / \mathrm{w}$ in isopropanol) and $600 \mu \mathrm{L}$ of ethanol and the suspension was sonicated for 1 hour. The Nafion catalyst ink was then pipetted onto the working electrode. When $\mathrm{CV}$ were acquired using a $3 \mathrm{~mm}$ working electrode, $2 \mu \mathrm{L}$ was drop cast onto the surface and allowed to stand in air until dry. In the case of the larger glassy carbon disk for the ring-disk electrode, $3 \mu \mathrm{L}$ were used to completely cover the surface. 


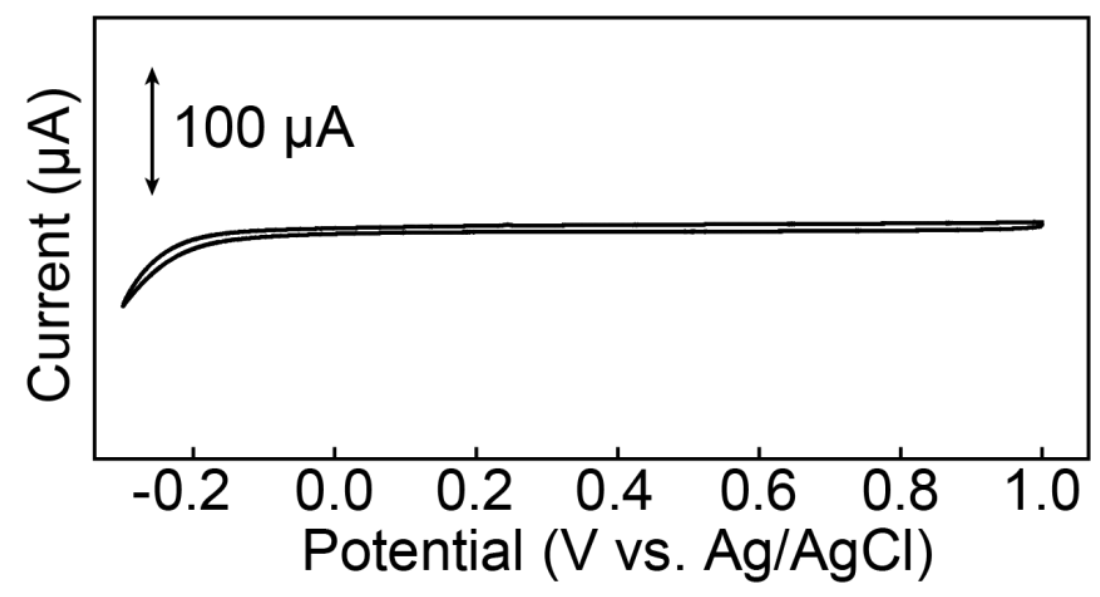

Figure S42. Carbon black blank. Film was made as outlined above; however, no catalyst was added. Solvent was $0.5 \mathrm{M} \mathrm{H}_{2} \mathrm{SO}_{4}$ and solution was sparged with $\mathrm{O}_{2}$ before $\mathrm{CV}$ was acquired. Scan rate: $100 \mathrm{mV} / \mathrm{sec}$.
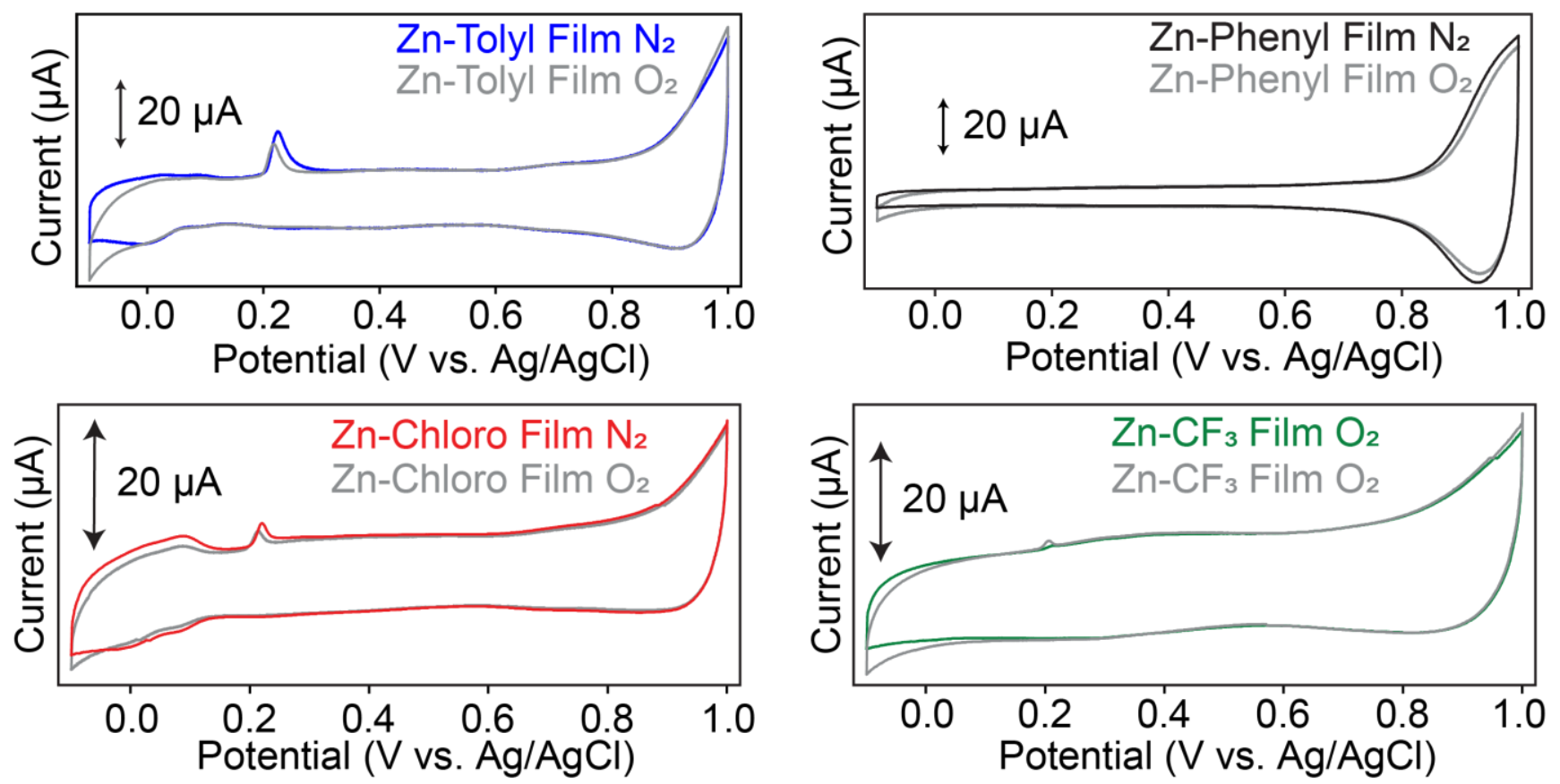

Figure S43. Comparing Zn-prisms immobilized in catalyst inks drop cast onto electrodes under inert and catalytic conditions. In general, the Zn-prism did not show any current enhancement under catalytic conditions.

\section{Calculation of Collection Efficiency}

The collection efficiency $(N)$ is a metric which relates the current at the disk electrode to the current at the ring electrode. It acts as a correction factor to account for compound (in this case) reduced at the disk electrode that does not get re-oxidized at the ring electrode which is set at a sufficiently oxidizing potential. ${ }^{9}$ Mathematically:

$$
N=\frac{-i_{R}}{i_{D}}
$$

Where $-i_{R}$ refers to the current at the ring electrode (upper trace in Figure $\mathbf{S 4 4}$ ) and $i_{D}$ refers to the disk current response (lower trace Figure S44). 


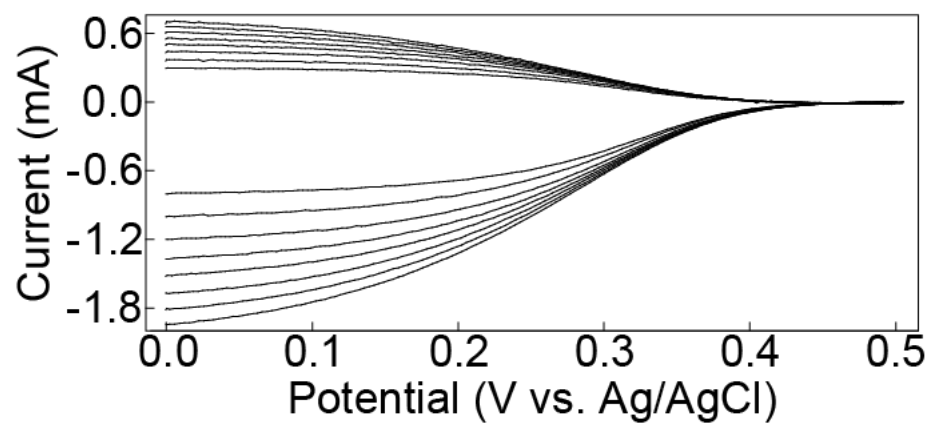

Figure S44. Experimental collection efficiency of the RRDE used for all catalytic experiments. Analyte: $10 \mathrm{mM} \mathrm{K}{ }_{3} \mathrm{Fe}(\mathrm{CN})_{6}$ Electrolyte: $1 \mathrm{M} \mathrm{KCl}$ in water. Rotation rates used: 500, 800, 1200, 1600, 2000, 2500, 3000, and $3500 \mathrm{rpm}$.

Faradaic Efficiency Determined using RRDE of Heterogeneous Catalyst Inks

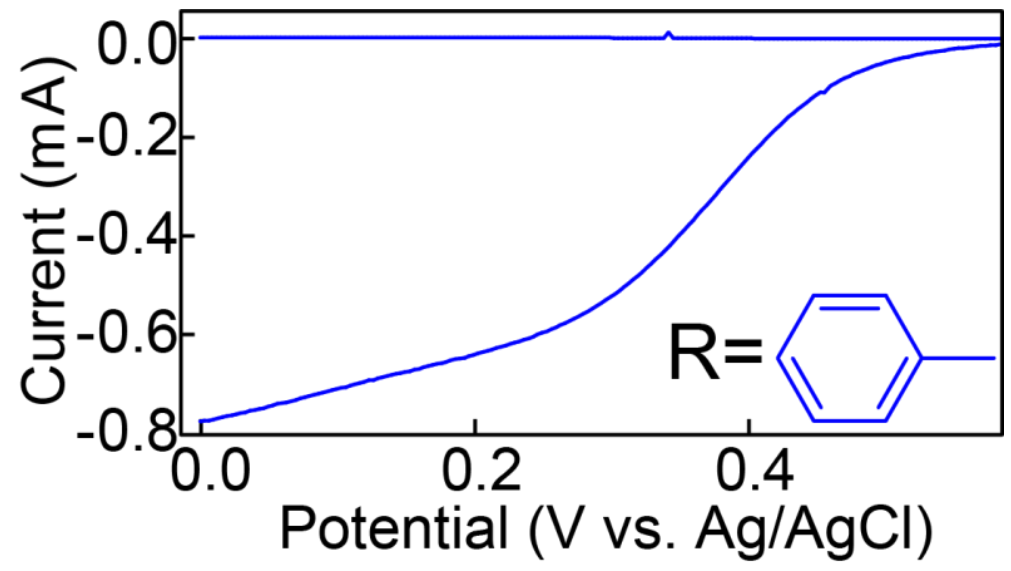

Figure S45. Hydrodynamic voltammogram of Co-Tolyl prism using RRDE. Lower trace represents modified-disk current where catalysis is occurring, upper trace is the current response of the Pt-ring electrode held a $+1.0 \mathrm{~V}$. Rotation rate was 2500 RPM in aqueous $0.5 \mathrm{M} \mathrm{H}_{2} \mathrm{SO}_{4}$. Very little current response was observed at the ring electrode suggesting high selectivity for $\mathrm{O}_{2}$ reduction to water. $\mathrm{Scan}$ rate: $20 \mathrm{mV} / \mathrm{sec}$.

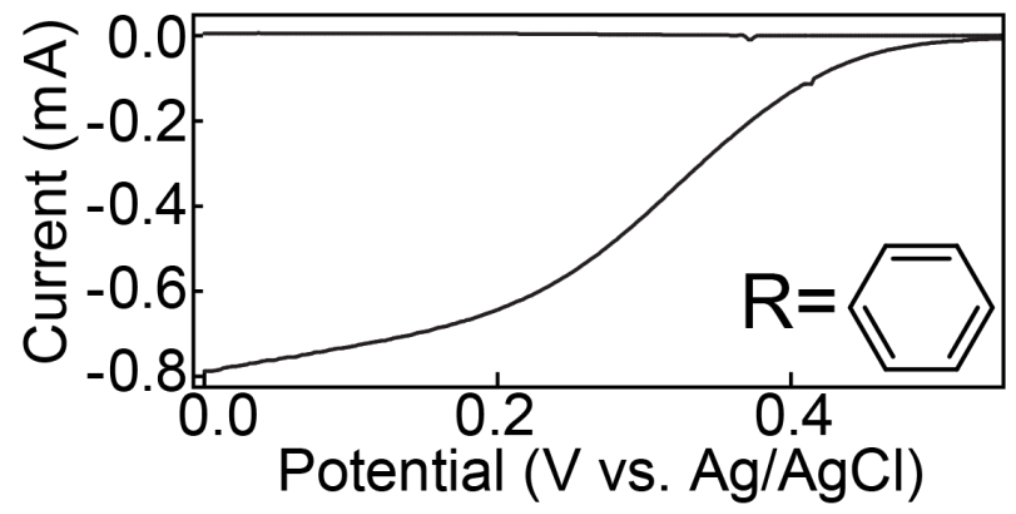

Figure S46. Hydrodynamic voltammogram of Co-Phenyl prism using RRDE. Lower trace represents modified-disk current where catalysis is occurring, upper trace is the current response of the Pt-ring electrode held a $+1.0 \mathrm{~V}$. Rotation rate was 2500 RPM in aqueous $0.5 \mathrm{M} \mathrm{H}_{2} \mathrm{SO}_{4}$. Scan rate: $20 \mathrm{mV} / \mathrm{sec}$. 


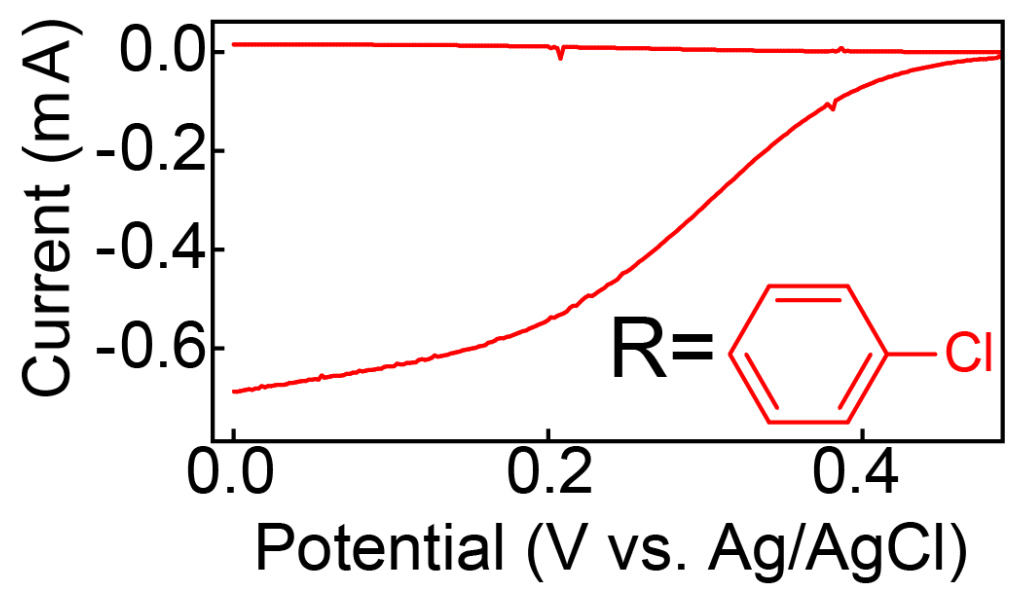

Figure S47. Hydrodynamic voltammogram of Co-Chloro prism using RRDE. Lower trace represents modified-disk current where catalysis is occurring, upper trace is the current response of the Pt-ring electrode held a $+1.0 \mathrm{~V}$. Rotation rate was 2500 $\mathrm{RPM}$ in aqueous $0.5 \mathrm{M} \mathrm{H}_{2} \mathrm{SO}_{4}$. Scan rate: $20 \mathrm{mV} / \mathrm{sec}$.

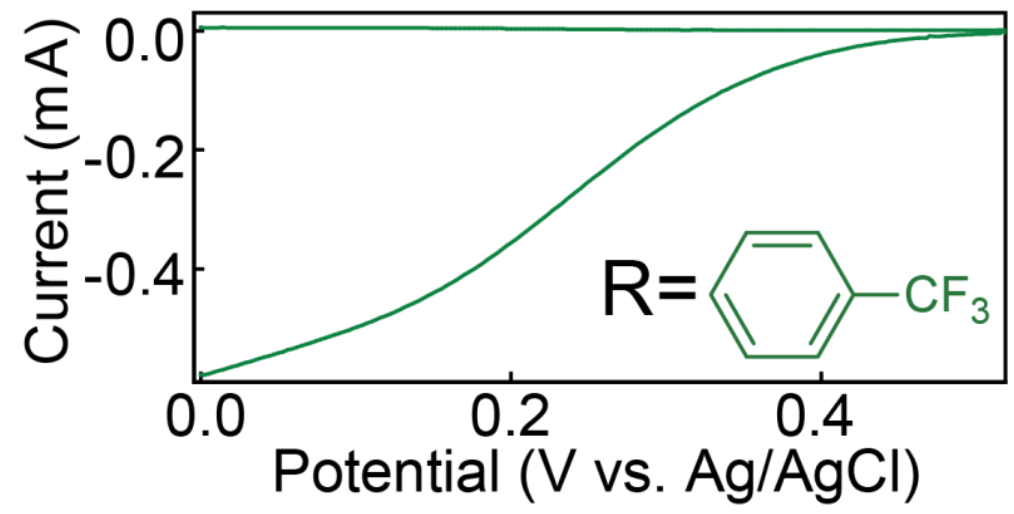

Figure S48. Hydrodynamic voltammogram of $\mathbf{C o -} \mathbf{C F}_{\mathbf{3}}$ prism using RRDE. Lower trace represents modified-disk current where catalysis is occurring, upper trace is the current response of the Pt-ring electrode held a $+1.0 \mathrm{~V}$. Rotation rate was 2500 $\mathrm{RPM}$ in aqueous $0.5 \mathrm{M} \mathrm{H}_{2} \mathrm{SO}_{4}$. Scan rate: $20 \mathrm{mV} / \mathrm{sec}$.

Koutecký-Levich Analysis

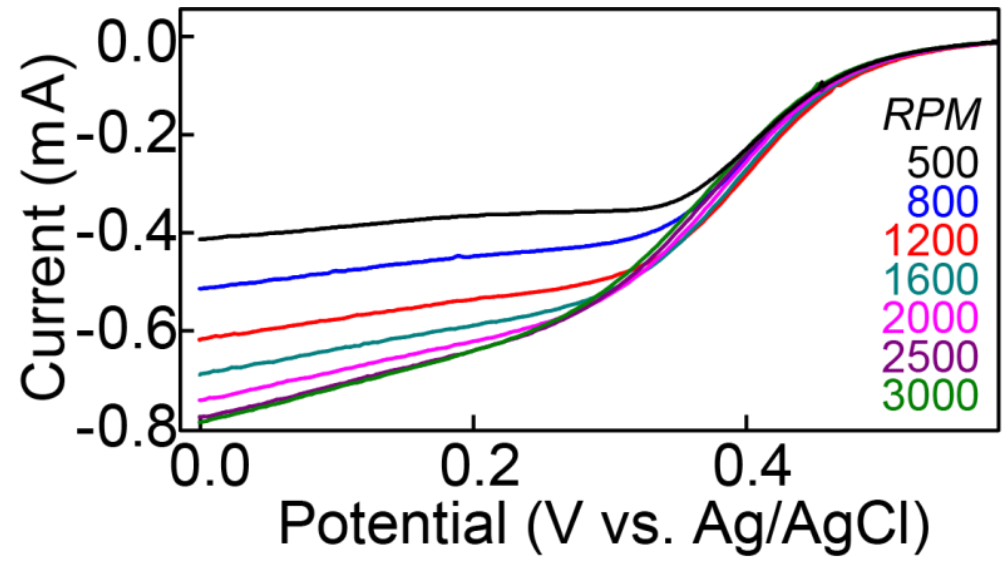

Figure S49. LSV of the Co-Tolyl prism using RDE electrode. Performed in $0.5 \mathrm{M} \mathrm{H}_{2} \mathrm{SO}_{4}$ at a scan rate of $20 \mathrm{mV} / \mathrm{sec}$ with a glassy carbon disc electrode coated in a catalyst ink. 


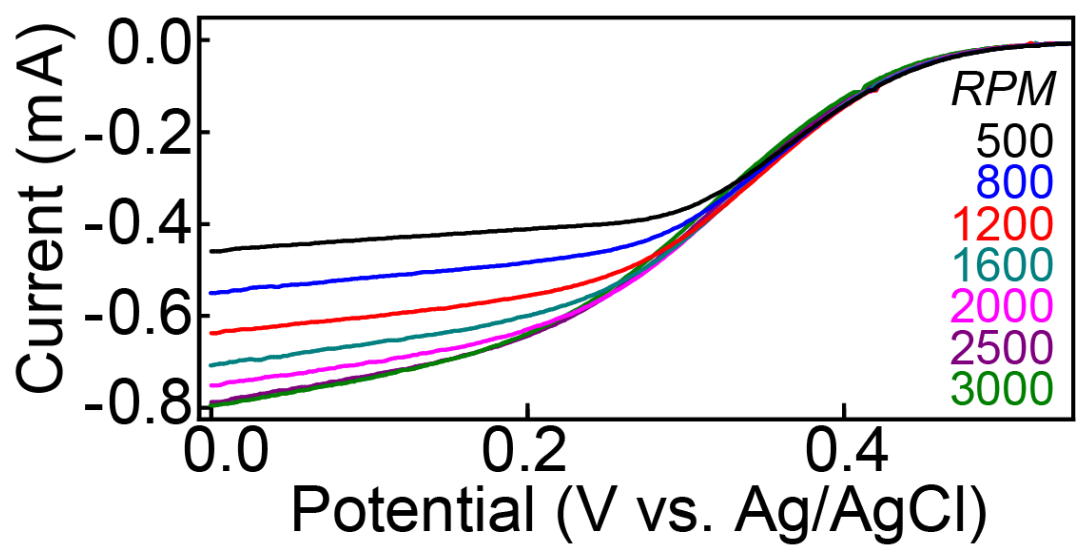

Figure S50. LSV of the Co-Phenyl prism using RDE electrode. Performed in $0.5 \mathrm{M} \mathrm{H}_{2} \mathrm{SO}_{4}$ at a scan rate of $20 \mathrm{mV} / \mathrm{sec}$ with a glassy carbon disc electrode coated in a catalyst ink.

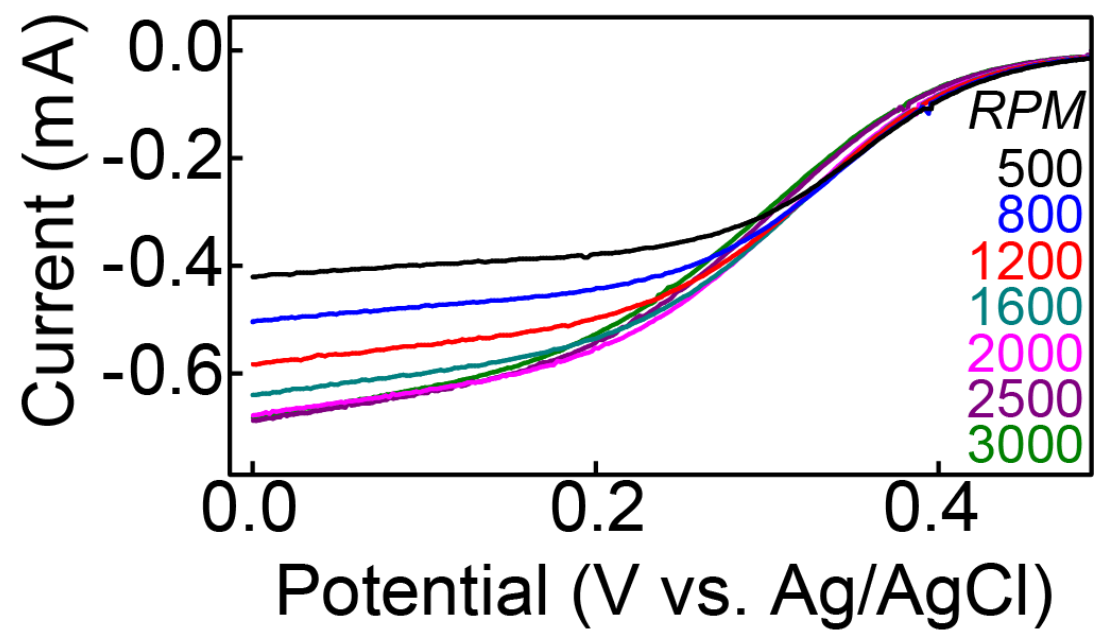

Figure S51. LSV of the Co-Chloro prism using RDE electrode. Performed in $0.5 \mathrm{M} \mathrm{H}_{2} \mathrm{SO}_{4}$ at a scan rate of $20 \mathrm{mV} / \mathrm{sec}$ with a glassy carbon disc electrode coated in a catalyst ink.

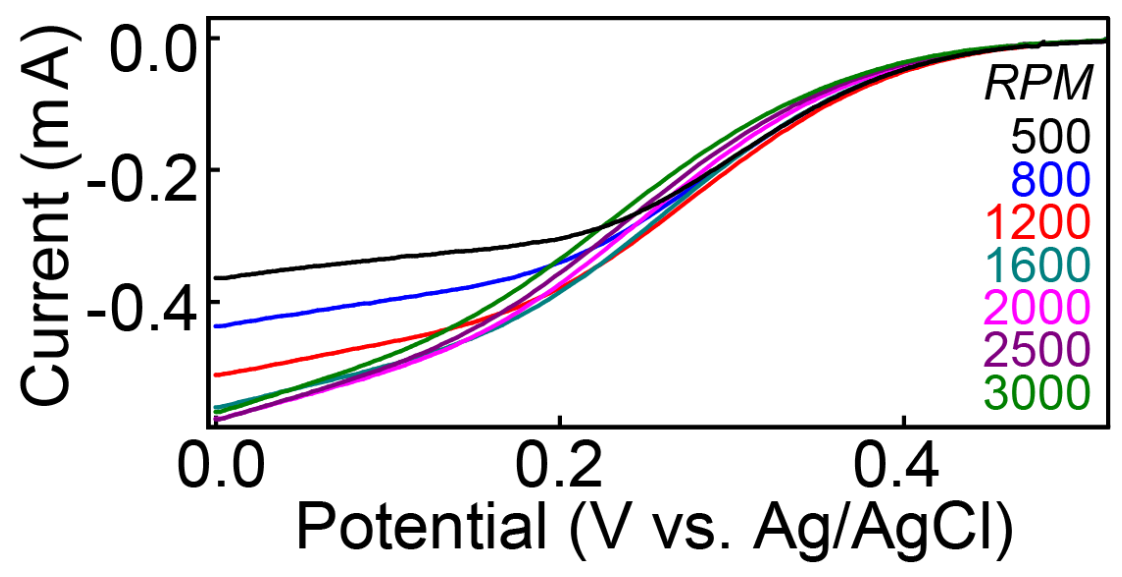

Figure S52. LSV of the Co- $\mathrm{CF}_{3}$ prism using RDE electrode. Performed in $0.5 \mathrm{M} \mathrm{H}_{2} \mathrm{SO}_{4}$ at a scan rate of $20 \mathrm{mV} / \mathrm{sec}$ with a glassy carbon disc electrode coated in a catalyst ink. 


$$
\frac{1}{i}=\frac{1}{B} \omega^{-\frac{1}{2}}+\frac{1}{i_{K}}
$$

Where $i$ is the current (A) response at the RDE. $B$ refers to the Levich constant, $\omega$ is the rotation rate $(\mathrm{rad} / \mathrm{sec})$, and $i_{K}(\mathrm{~A})$ is the kinetic current, the current absent any mass transport effects (i.e. the concentration of substrate in the electrochemical double layer is equal to bulk substrate concentration). Examining the current response within diffusion-controlled region (the plateaued region of an LSV obtained using an RDE) at several different overpotentials $(\eta)$ plots of $1 / i$ vs. $\omega^{-1 / 2}$ allows

$$
i_{K}=n F A k_{\text {het }}\left[O_{2}\right] \Gamma_{\text {cat }}
$$

Equation S3 allows for the kinetic current to be related to the heterogeneous electron transfer rate constant $\left(k_{h e t}\right)$. The other variables are: $n$ is the number of electrons transferred in the electrochemical process, $F$ is Faraday's constant (96485 C/mol), $A$ is the electrode surface area $\left(0.95 \mathrm{~cm}^{2}\right),\left[\mathrm{O}_{2}\right]$ is the concentration of $\mathrm{O}_{2}\left(1.1 \cdot 10^{-6} \mathrm{~mol} / \mathrm{cm}^{3}\right.$ in $\left.0.5 \mathrm{M} \mathrm{H}_{2} \mathrm{SO}_{4}\right)$, and $\Gamma_{\text {cat }}$ is the "ideal active site density" (i.e. mole of catalyst per unit area of the electrode, in $\mathrm{mol} / \mathrm{cm}^{2}$ ). To experimentally determine the number of electrons transferred, equation S4 was used.

$$
n=4-2\left(\frac{\% \mathrm{H}_{2} \mathrm{O}_{2}}{100}\right)
$$

Where the $\% \mathrm{H}_{2} \mathrm{O}_{2}$ was determined from Equation 1 (Main Text). Finally, $k_{\text {het }}$ can be related to the standard rate constant $\left(k_{s}\right)$ for the electrochemical process using the following equation (S5):

$$
k_{\text {het }}=k_{s} e^{\frac{-\alpha \eta}{R T}}
$$

Where $\alpha$ is the transfer coefficient, $R$ is the gas constant $(8.413 \mathrm{~J} / \mathrm{mol} \cdot \mathrm{K})$ and $T$ is the temperature $(298 \mathrm{~K})$.

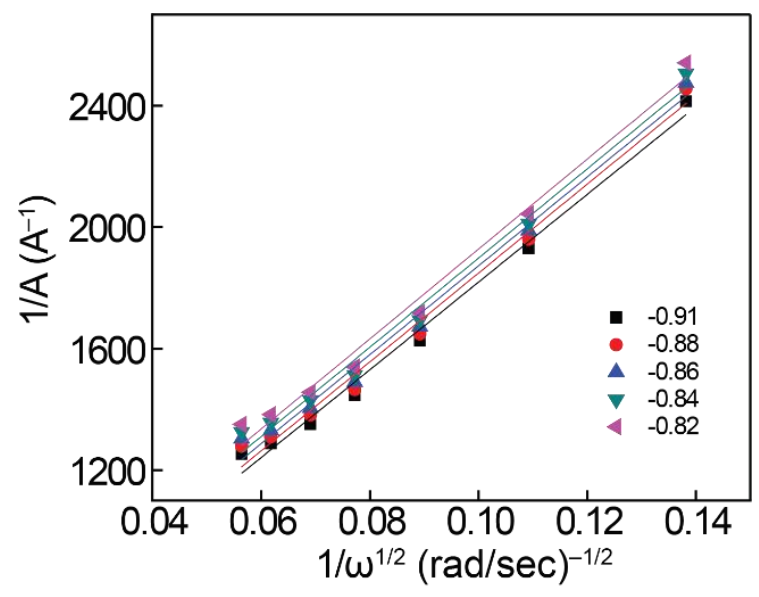

Figure S53. Plot of $1 /$ A vs. $\omega^{-1 / 2}$ at different overpotentials (V) used to determine $i_{K}$ using Eq. S2 and S3 for the Co-Tolyl prism.

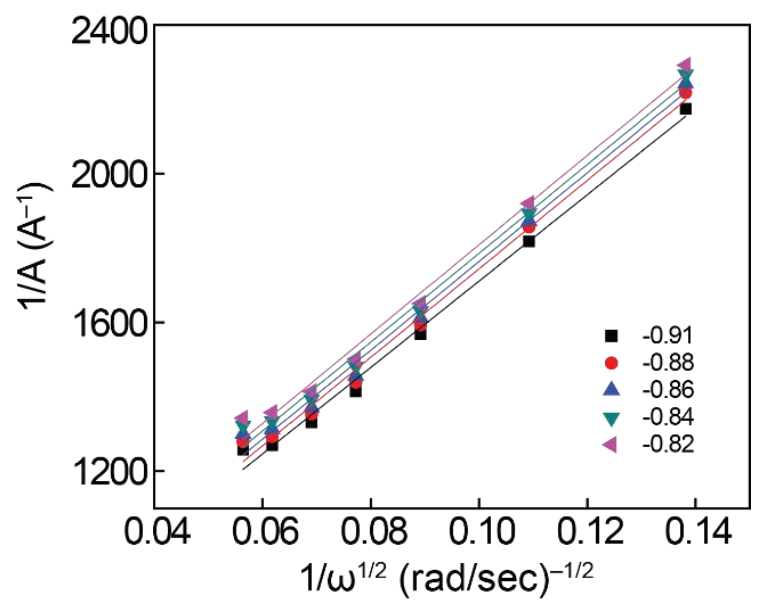

Figure S54. Plot of $1 / \mathrm{A}$ vs. $\omega^{-1 / 2}$ at different overpotentials (V) used to determine $\mathrm{i}_{\mathrm{K}}$ using Eq. $\mathrm{S} 2$ and $\mathrm{S} 3$ for the Co-Phenyl prism. 


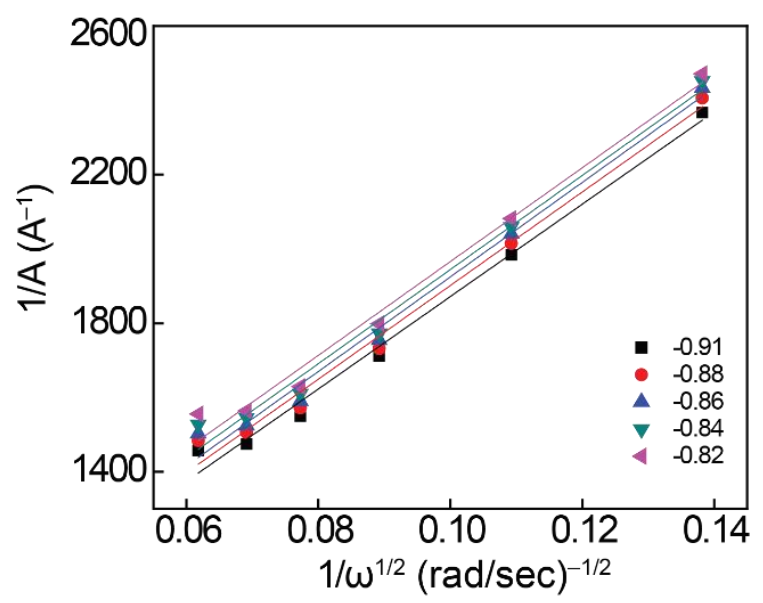

Figure S55. Plot of 1/A vs. $\omega^{-1 / 2}$ at different overpotentials (V) used to determine $\mathrm{i}_{\mathrm{K}}$ using Eq. S2 and S3 for the Co-Chloro prism.

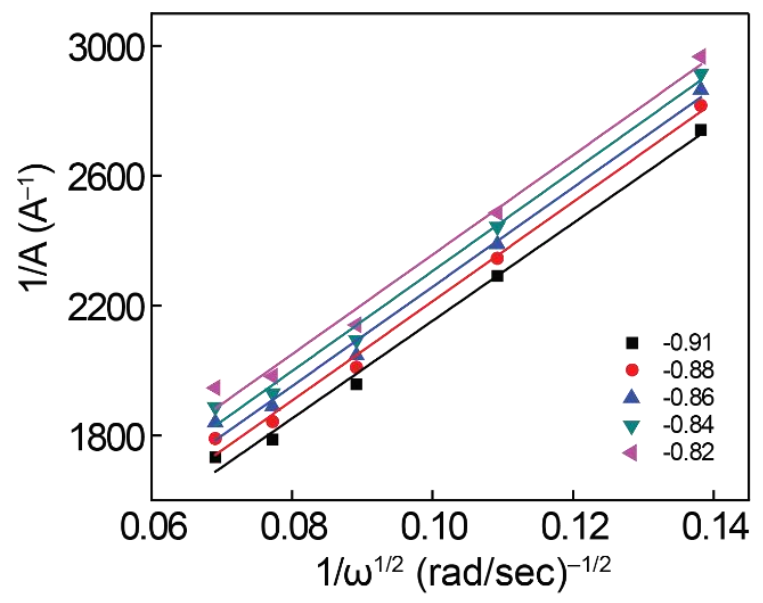

Figure S56. Plot of 1/A vs. $\omega^{-1 / 2}$ at different overpotentials (V) used to determine $i_{\mathrm{K}}$ using Eq. S2 and S3 for the Co-CF 3 prism.

The above plots (Figures S48-51) represent the inverse of current at different rotations rates at 5 different overpotentials. It can be seen from Equation S2 that the y-intercept of the linear fit will be 1/iK. Using Equation S3 the heterogeneous electron transfer rate constant $\left(k_{\text {het }}\right)$ can be determined from $i_{K}$. Using the observed $k_{\text {het }}$ at different overpotentials $(\eta)$ and equation S5, one can solve for $k_{s}$, the standard rate constant, using a plot of $\ln \left(k_{h e t}\right)$ vs. $\eta$ in which the y-intercept will be $\ln \left(k_{s}\right)$ which can be seen below. 


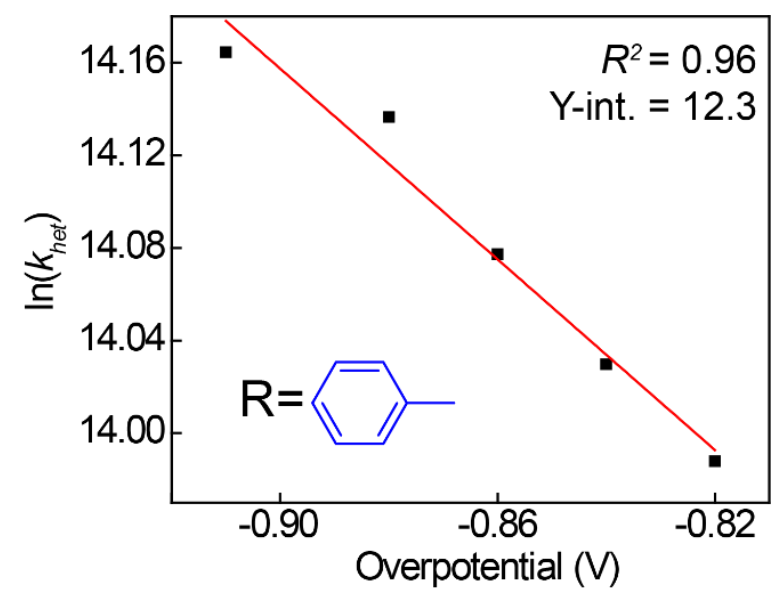

Figure S57. Plot of $\ln \left(\mathrm{k}_{\mathrm{het}}\right)$ vs. overpotential for the Co-Tolyl prism. Each data point was determined using the y-intercept at different overpotentials from Figure S53. The y-intercept of this plot is $\ln \left(\mathrm{k}_{\mathrm{s}}\right)$.

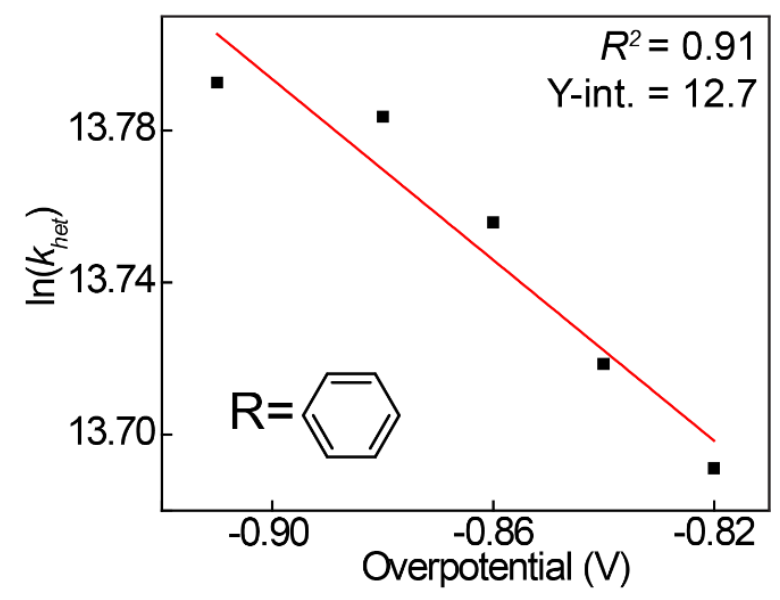

Figure S58. Plot of $\ln \left(\mathrm{k}_{\mathrm{het}}\right)$ vs. overpotential for the Co-Phenyl prism. Each data point was determined using the y-intercept at different overpotentials from Figure S54. The y-intercept of this plot is $\ln \left(\mathrm{k}_{\mathrm{s}}\right)$.

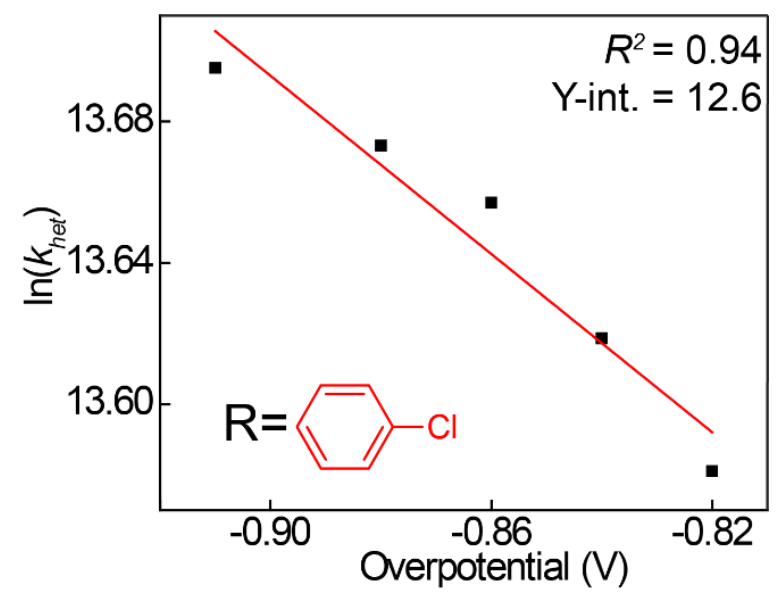

Figure S59. Plot of $\ln \left(\mathrm{k}_{\mathrm{het}}\right)$ vs. overpotential for the Co-Chloro prism. Each data point was determined using the y-intercept at different overpotentials from Figure S55. The y-intercept of this plot is $\ln \left(\mathrm{k}_{\mathrm{s}}\right)$. 


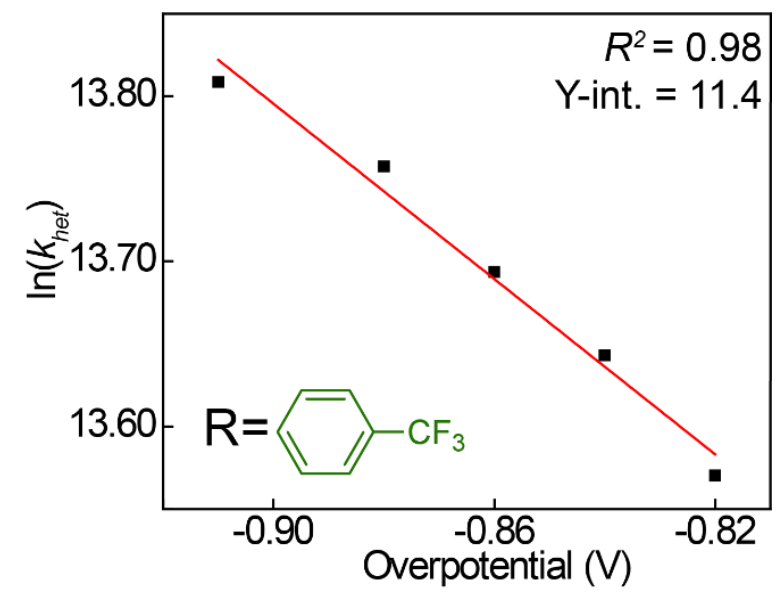

Figure S60. Plot of $\ln \left(\mathrm{k}_{\text {het }}\right)$ vs. overpotential for the $\mathbf{C o -} \mathbf{C F}_{\mathbf{3}}$ prism. Each data point was determined using the y-intercept at different overpotentials from Figure S56. The $y$-intercept of this plot is $\ln \left(\mathrm{k}_{\mathrm{s}}\right)$.

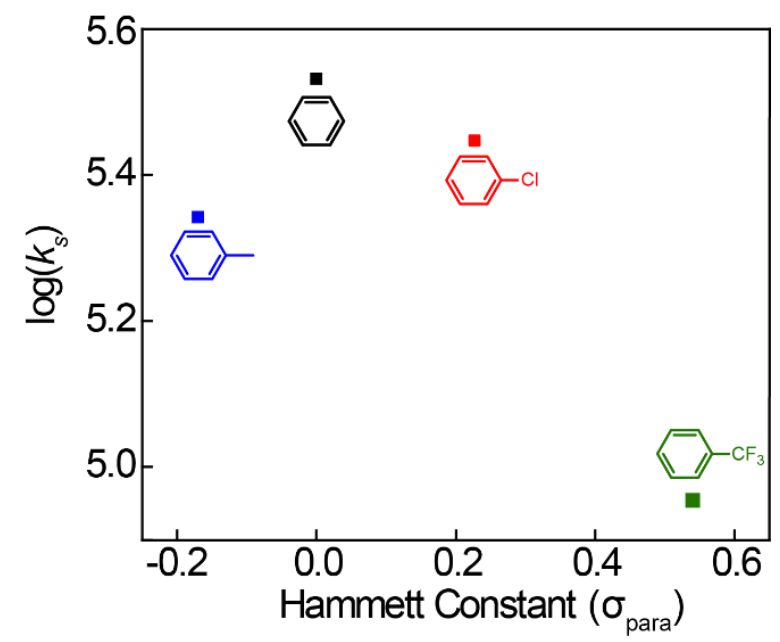

Figure S61. Plot of $\log \left(\mathrm{k}_{\mathrm{s}}\right)$ vs Hammett substituent constant. No linear free-energy relationship was observed, this implies that the observed modulation in the standard electrochemical rate constant is not purely due to an electronic effect, suggesting a steric component is also involved.

\section{Tafel Slope Analysis}

The Tafel slope $(b)$ can be used to inform on mechanistic shifts. The Tafel equation (S6) is defined as 9 :

$$
\eta=a+b \times \log (j)
$$

Where $a$ is the exchange current density and $j$ is the current density. The Tafel slope can be determined graphically from the linear region of the Tafel plots seen below. 


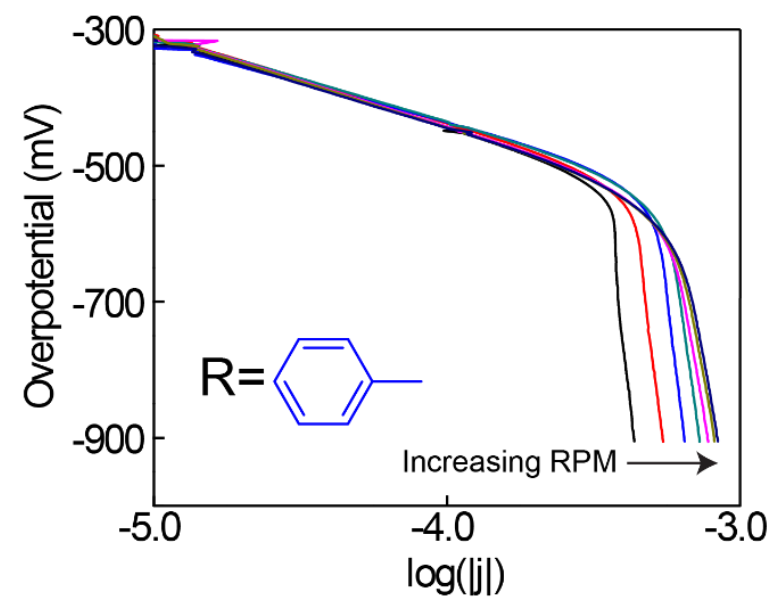

Figure S62. Co-Tolyl prism Tafel plot slope of -130(7) mV/decade

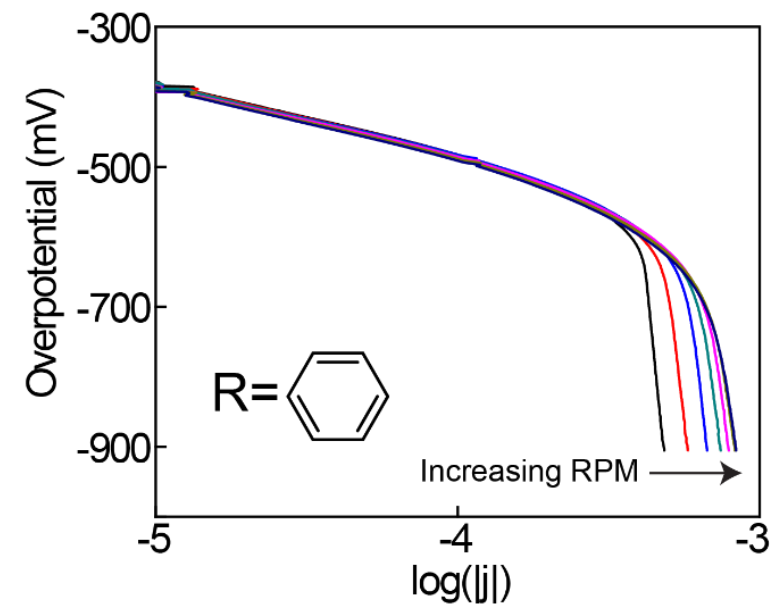

Figure S63. Co-Phenyl prism Tafel plot slope of -101(2) mV/decade

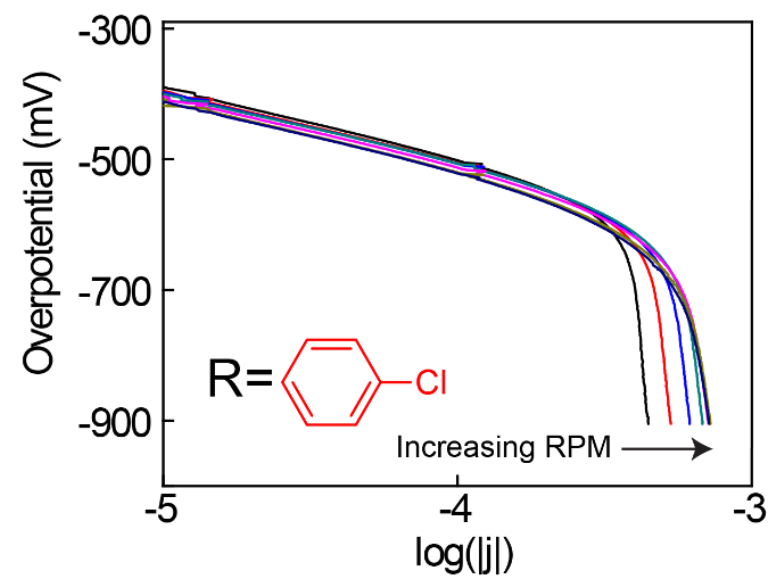

Figure S64. Co-Chloro prism Tafel plot, slope of -128(2) mV/decade 


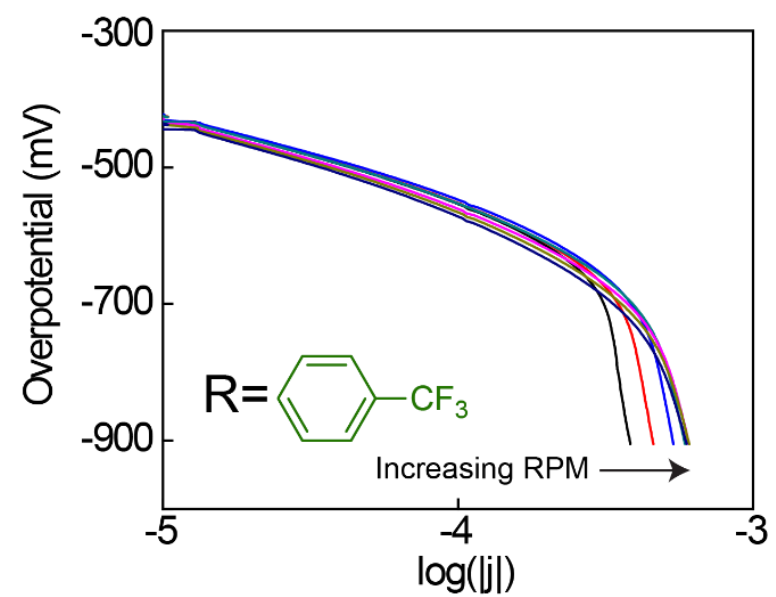

Figure S65. Co-CF 3 prism Tafel plot, slope of -120(3) $\mathrm{mV} / \mathrm{dec} a d e$.

\section{References}

1. Zhang, W.-Z.; Han, Y.-F.; Lin, Y.-J.; Jin, G.-X. Synthesis and characterization of molecular rectangles of half-sandwich p-cymene ruthenium complexes bearing oxamidato ligands. Dalton Trans. 2009, 8426-8431.

2. Nakazono, T.; Parent, A. R.; Sakai, K. Cobalt porphyrins as homogeneous catalysts for water oxidation. Chem. Commun. 2013, 49, 6325-6327.

Bruker APEX3, Bruker-Nonius AXS Inc.: Madison, Wisconsin, USA., 2016.

Dolomanov, O. V.; Bourhis, L. J.; Gildea, R. J.; Howard, J. A. K.; Puschmann, H. OLEX2: a complete structure solution, refinement and analysis program. J. Appl. Crystallogr. 2009, 42, 339-341.

5. Sheldrick, G. A short history of SHELX. Acta Crystallographica Section A 2008, 64, 112-122.

6. Sheldrick, G. Crystal structure refinement with SHELXL. Acta Crystallographica Section C 2015, 71, 3-8.

7. Sheldrick, G. SHELXT - Integrated space-group and crystal-structure determination. Acta Crystallographica Section A 2015, 71, 3-8.

8. Guzei, I. An idealized molecular geometry library for refinement of poorly behaved molecular fragments with constraints. J. Appl. Crystallogr. 2014, 47, 806-809.

9. Bard, A. J.; Faulkner, L. R., Electrochemical Methods: Fundamentals and Applications. Wiley: 2000. 